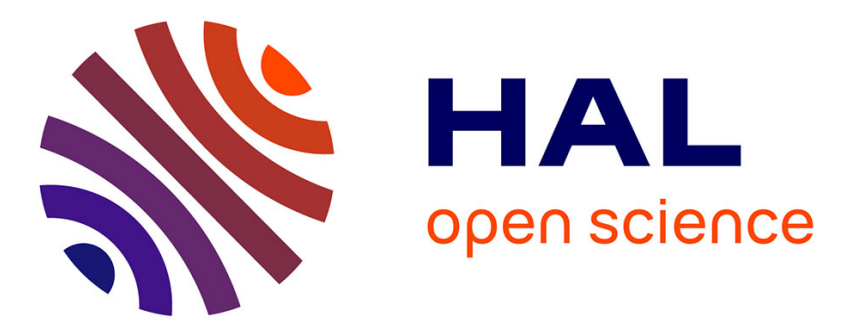

\title{
Solar Tides in the Middle and Upper Atmosphere of Mars
}

\author{
Jeffrey Forbes, Xiaoli Zhang, Francois Forget, Ehouarn Millour, Armin
}

Kleinböhl

\section{- To cite this version:}

Jeffrey Forbes, Xiaoli Zhang, Francois Forget, Ehouarn Millour, Armin Kleinböhl. Solar Tides in the Middle and Upper Atmosphere of Mars. Journal of Geophysical Research Space Physics, 2020, 125 (9), pp.e2020JA028140. 10.1029/2020JA028140 . hal-03103958

\section{HAL Id: hal-03103958 https://hal.science/hal-03103958}

Submitted on 8 Jan 2021

HAL is a multi-disciplinary open access archive for the deposit and dissemination of scientific research documents, whether they are published or not. The documents may come from teaching and research institutions in France or abroad, or from public or private research centers.
L'archive ouverte pluridisciplinaire HAL, est destinée au dépôt et à la diffusion de documents scientifiques de niveau recherche, publiés ou non, émanant des établissements d'enseignement et de recherche français ou étrangers, des laboratoires publics ou privés. 
1 Solar Tides in the Middle and Upper Atmosphere of Mars

$$
8
$$

Jeffrey M. Forbes ${ }^{1}$, Xiaoli Zhang ${ }^{1}$, Francois Forget $^{2}$, Ehouarn Millour ${ }^{2}$, and

\author{
Armin Kleinböhl ${ }^{3}$
}

$5 \quad{ }^{1}$ Ann and H.J. Smead Department of Aerospace Engineering Sciences, University of Colorado, Boulder,

9

Key Points:

- Multi-year latitude vs. Ls climatology of Mars tidal spectrum is derived from MRO/MCS $12 \quad$ measurements at $76 \mathrm{~km}$.

${ }_{13}$ - MCD tidal spectrum compares well with MCS climatology, and with longitude struc-

${ }_{14}$ tures in MGS aerobraking densities near $112 \mathrm{~km}$.

15 - MCD latitude-height tidal structures are strongly influenced by mean winds and

16 dissipation, and provide new insights into density variability at 100-170 km alti-

$17 \quad$ tude.
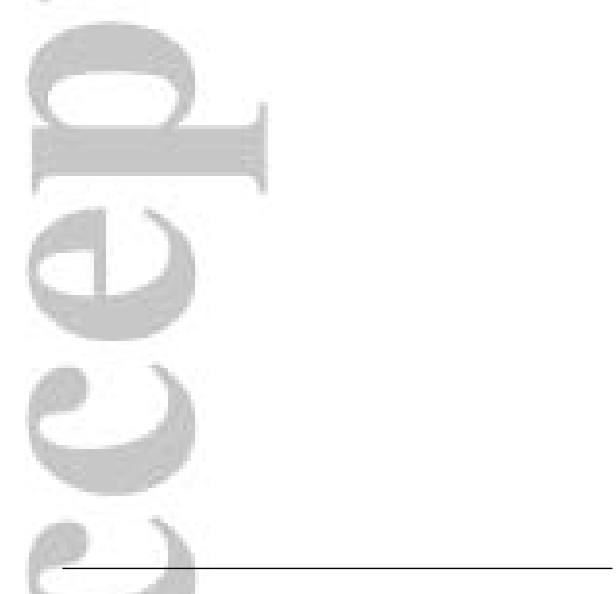

Corresponding author: Jeffrey M. Forbes, forbes@colorado.edu

This article has been accepted for publication and undergone full peer review but has not been through the copyediting, typesetting, paginlation and proofreading process which may lead to differences between this version and the Version of Record. Please cite this article as doi: 10.1029/2020-\$प्र००० 


\section{Abstract}

Solar tides are responsible for much of the spatial-temporal variability of Mars' upper atmosphere (100 - 200 km). However, the tidal spectrum, its latitude versus Ls variability, and its vertical evolution remain uncertain. In this paper, Mars Climate Sounder (MCS) temperature measurements at $76 \mathrm{~km}$ above Mars' areoid are used to construct a multi-year latitude versus Ls climatology of the tidal spectrum. The most important spectral components include the solar-synchronous ("migrating") components DW1, SW2, and the solar-asynchronous ("non-migrating") tides DE3, DE2, DE1, SE1, S0, and SW1. The Mars Climate Database (MCD), which provides predictions from the Laboratoire de Météorologie Dynamique (LMD) Global Climate Model, captures particularly well the amplitudes and key structural features of the solar-asynchronous tides at $76 \mathrm{~km}$ that furthermore underly the large longitudinal structures in density that are observed between 100-200 km. Height-latitude and latitude-Ls structures of MCD density perturbations are therefore examined between $76-172 \mathrm{~km}$ and interpreted in terms of mean wind and dissipation effects. In particular, due to the smaller radius and more intense zonalmean zonal winds at Mars compared to Earth, Doppler-shift effects are significantly exaggerated compared to Earth. Evidence is also provided for non-negligible contributions to density variability from stationary planetary waves which arise from tide-tide nonlinear interactions. It is moreover shown that MCD captures the salient amplitude and phase characteristics of the $\sim \pm 30-60 \%$ longitudinal density perturbations measured by the Mars Global Surveyor (MGS) accelerometer. This, and the excellent MCD-MCS agreement at $76 \mathrm{~km}$, lends credibility to the ability of MCD to provide new insights into thermosphere density variability at Mars due to vertical coupling by solar tides.

\section{Introduction}

The importance of solar-driven tides to the dynamics of Mars' atmosphere is now common knowledge. Due to their vertical propagation characteristics and growth with height, they serve the important role of transmitting the variability associated with their lower-atmosphere sources and other meteorological processes to much higher altitudes (e.g., Angelats i Coll et al., 2004; Forbes et al., 2002; Moudden and Forbes, 2008a; England et al., 2016, 2019), even to the edge of space (i.e., the exobase, roughly near $200 \mathrm{~km}$ ). Additionally, their dissipation above about $80 \mathrm{~km}$ altitude deposits net momentum, thus contributing as a driver for the mean circulation in this altitude region (e.g., Moudden 
tude, so that solar tides are expressed mathematically as

$$
\sum_{s} \sum_{n} A_{n, s}(z, \theta)\left[\cos \left(n \Omega t+s \lambda-\phi_{n, s}(z, \theta)\right]\right.
$$

and Forbes, 2008b), and in turn redistributing minor chemical constituents including water (Shaposhnikov et al., 2019).

$\mathrm{CO}_{2}$ near the surface, radiation absorption by dust and water ice clouds, and near-infrared radiation absorption by $\mathrm{CO}_{2}$ above $70 \mathrm{~km}$. These processes are strongly influenced by topography, thermal properties of the surface, and by the dynamical processes responsible for redistributing the absorbing species. Consequently, both the solar heating and the atmospheric responses are dependent on latitude, longitude, local solar time (LST) and day of year. Throughout this paper, LST refers to local true solar time.

Planetary rotation furthermore admits periodic dependencies on time and longi-

where $\mathrm{t}=$ universal time $(\mathrm{UT}), \Omega=2 \pi \mathrm{sol}^{-1}, \mathrm{z}=$ altitude, $\theta=$ latitude, integer $s$ is the zonal wavenumber, integer $\mathrm{n}$ defines the frequency or period of the oscillation, $A_{n, s}$ is the amplitude, and $\phi_{n, s}$ is the phase (i.e., longitude $\lambda$ of maximum at $0000 \mathrm{UT}$, or time of maximum at $\lambda=0$ ). The outer sum captures the longitude dependence of any tidal frequency (either source or response), while the inner sum captures the diurnal variation of the source (or response) at any given longitude. Setting the quantity in parentheses equal to zero and taking the derivative defines the zonal phase speed of any tidal component: $C_{p h}=\frac{-n \Omega}{s}$. For the assumed mathematical expression (1), this implies $s<0$ for eastward-propagating waves $\left(C_{p h}>0\right)$ and $s>0$ for westward-propagating $\left(C_{p h}<0\right)$ waves. Tides with $s=0$ do not propagate zonally and are called zonallysymmetric; this means that the atmosphere oscillates at a given tidal frequency in unison at all longitudes. Note also that if $s=n$, then a tide with any frequency migrates westward with the apparent phase speed of the Sun to a ground-based observer; these are solar-synchronous and often referred to as "migrating" tides. Tides with $s \neq n$ are solar asynchronous and often referred to as "non-migrating" tides, and it is these tides that capture the longitude dependence of the tidal response (or source).

The notation DWs or DEs is used to denote a westward or eastward-propagating diurnal tide, respectively, with zonal wavenumber $=\mathrm{s}$. For semidiurnal oscillations, $\mathrm{S}$ replaces D. The zonally-symmetric oscillations are denoted D0, S0. In the present pa- 
per we will confine ourselves to the diurnal $(n=1)$ and semidiurnal $(n=2)$ solar tides, which generally exceed higher-order tides in magnitude and importance.

Much of what we know, observationally, about tides in the middle atmosphere $(\sim 50$ $100 \mathrm{~km}$ ) of Mars has been learned from temperature measurements by the Mars Climate Sounder (MCS) instrument (McCleese et al., 2007) on Mars Reconnaissance Orbiter (MRO), which has been observing the atmosphere and surface of Mars since September 2006. (However, see also observations of Mars solar tides between 70-110 km by the SPICAM instrument on Mars Express reported in Withers et al., 2011). MCS temperature measurements extend from near the surface to about $80 \mathrm{~km}$ altitude between $\pm 87^{\circ}$ latitude. In its original in-track observing mode, one of the difficulties attached to the MCS measurements, insofar as tides are concerned, is the limited LST coverage offered by the $\sim 0300 / 1500$ LST solar-synchronous orbit of MRO. Expressing (1) in terms of local time $\left(t_{L S T}\right)$,

$$
\sum_{s} \sum_{n} A_{n, s}(z, \theta)\left[\cos \left(n \Omega t_{L S T}+(s-n) \lambda-\phi_{n, s}(z, \theta)\right]\right.
$$

Expression (2) demonstrates that the signatures of tides in solar-synchronous $\left(t_{L S T} \approx\right.$ constant) satellite data are identifiable in terms their longitude structure, specifically their "space-based wavenumber" $k_{s}=|s-n|$. However, there is ambiguity inherent in these signatures, since multiple combinations of $s$ and $n$ yield the same $k_{s}$. The first tidal analyses of MCS temperatures using the in-track observing mode (Lee et al., 2009; Guzewich et al., 2012) exploited the fact that the ascending and descending parts of the orbits are 12 hours apart (equatorward of $\pm 75^{\circ}$ latitude). Since tides with $n$ odd are in anti-phase at local times 12 hours apart, the difference between ascending and descending observations is expected to contain only odd tides (diurnal, terdiurnal, etc.) and the sum is expected to contain only even ones (semidiurnal, quatradiurnal, etc., as well as the $n=$ 0 zonal mean and stationary planetary waves (SPW)). Assuming that higher-order tides are negligible relative to diurnal and semidiurnal tides, spectral analysis can then be applied to the resulting sum and difference fields to reveal the amplitudes of the diurnal or semidiurnal tides contributing to each solar-synchronous zonal wave number, $k_{s}$. For a given $n$ and $k_{s}=|s-n|$, there are generally two mathematical solutions, and a more physically reasonable can often be identified. These authors presented evidence for DE3, DE2, DE1, D0, DW1, and SE1 tides, as well as SPW with $s=1$ and $s=2$ (hereafter SPW1, SPW2, respectively). 
Starting September, 2010, a special cross-track observing mode was also initiated which expanded the local time coverage (Kleinböhl et al., 2013). Kleinböhl et al. (2013) and Wu et al. (2015) exploited this capability, and were able to derive both diurnal and semidiurnal tides by directly fitting the MCS temperature measurements with respect to LST and longitude. Kleinböhl et al. (2013) provided the first delineation of the SW2 semidiurnal tide in Mars' middle atmosphere, and through comparisons with a Mars GCM, concluded that SW2 is a dominant feature throughout the Martian year, and that radiatively active water ice clouds (Wilson et al., 2007; 2008; see also Wilson, 2014) provide the most plausible explanation for the observed amplitudes. Wu et al. (2015) provided a new and expanded view of middle atmosphere tides, focusing on vertical tidal structures in the middle atmosphere at discrete latitudes and solar longitudes (Ls). Their study also included DW2, DW3 and SW1 in addition the aforementioned tidal components. These latter tides were hypothesized to originate from nonlinear interactions between solar-synchronous $(s=n)$ tides and SPWs.

At higher altitudes (100-200 km), information on tides has been inferred indirectly from longitude structures revealed in density measurements made by accelerometers on the Mars Global Surveyor (MGS), Mars Odyssey (MO), and Mars Reconnaissance Orbiter (MRO) satellites (e.g., Wang et al., 2006; Wilson, 2002; Withers, 2006; Withers et al., 2003), or more recently, in neutral mass spectrometer (e.g., England et al., 2016; Liu et al., 2017) and ultraviolet imager (e.g., England et al., 2016; Lo et al., 2015; Gröller et al., 2018) measurements on the MAVEN (Mars Atmosphere and Volatile Evolution) and Mars Express (Withers et al., 2011) missions. These studies have been based on either ascending or descending orbital data alone, and thus did not involve any constraints insofar as LST is concerned, in contrast to the aforementioned MCS tidal studies. In a few isolated cases where LST changed sufficiently fast over restricted ranges of latitude and Ls, more definitive tidal inferences have been made (e.g., England et al., 2019; Forbes et al., 2004; Forbes and Zhang, 2018).

Modeling frameworks for interpretation of the aforementioned data in terms of tidal coupling between the middle $(\sim 50-100 \mathrm{~km})$ and upper $(\sim 100-200 \mathrm{~km})$ atmosphere of Mars have been relatively few and incomplete. Forbes et al. (2002) used a linear model to extend several solar-asynchronous tidal fields at $70 \mathrm{~km}$ from the Ames General Circulation Model to $200 \mathrm{~km}$ altitude for $\mathrm{Ls}=30^{\circ}$ and $\mathrm{Ls}=270^{\circ}$. Angelats i Coll et al. (2004) and Moudden and Forbes (2008a) used GCMs to study the full spectrum of solar-asynchronous 
tides up to $120 \mathrm{~km}$ and $160 \mathrm{~km}$, respectively, for $\mathrm{Ls} \approx 75^{\circ}$, and both obtained some reasonable agreement with longitude structures of density from MGS accelerometer measurements. While Mars GCMs now exist that extend from the surface to Mars exosphere at $200 \mathrm{~km}$ (e.g., Bougher et al., 2011, 2015; Gonzalez-Galindo et al., 2009, 2013, 2015), they have not yet provided a more complete characterization and interpretation of vertical tidal coupling between Mars' middle and upper atmospheres.

By employing MCS temperature observations at $76 \mathrm{~km}$ altitude coupled with a general circulation model of Mars' atmosphere extending to ca. $200 \mathrm{~km}$, the objective of the present study is to significantly advance our understanding of how the tidal spectrum serves to couple the middle and upper atmospheres of Mars. Towards this end, we provide a climatological representation of the MCS tidal spectrum at $76 \mathrm{~km}$ above Mars' areoid from nearly pole to pole and as a continuous function of Ls. The veracity of the Mars Climate Database (MCD) derived from the LMD Global Climate Model (Forget et al., 1999; Millour et al., 2018; Gonzalez-Galindo et al., 2015, and references therein) is demonstrated against this tidal climatology, and the MCD is then applied to clarify how this tidal spectrum evolves with height. A specific goal is to shed light on the tidal origins of longitude variability in atmospheric total mass densities as observed by satelliteborne accelerometers during aerobraking operations (e.g., MGS, MRO, MO), as well as measurements from the MAVEN mission at higher altitudes $(\sim 135-200 \mathrm{~km})$.

The following section describes the MCS data that are utilized, and how they are processed to extract diurnal and semidiurnal tides. Section 3 provides results, which include an MCD-MCS comparison of tidal spectra as a function of latitude and Ls at 76 $\mathrm{km}$ altitude, examination and assessment of MCD vertical tidal structures, and MCD predictions of total mass density variations at 108 and $172 \mathrm{~km}$. A summary of results and conclusions comprises Section 4 .

\section{MCS Data and Processing, and MCD Model Description}

\subsection{Data employed}

The data used in this study consist of temperature profiles (version 5; Kleinböhl et al., 2009, 2011, 2017) from the MCS instrument on board MRO, and were obtained from the Planetary Atmospheres Node of the Planetary Data System (PDS) (http://pdsatmospheres.nmsu.edu/). MCS is a limb- and on-planet scanning infrared radiometer 
that launched aboard MRO and became fully operational at the end of September 2006 (McCleese et al., 2007). Temperature retrievals use the $\mathrm{CO}_{2}$ absorption feature at $15 \mathrm{mi}-$ cron and typically provide profile information from the near-surface up to $\sim 80 \mathrm{~km}$ altitude with a vertical resolution of $\sim 5 \mathrm{~km}$. MROs orbit is nearly polar and solar-synchronous with an inclination of $92.66^{\circ}$ and orbital period of 112 minutes. At any given time the spacecrafts local solar time (LST) is near 1500 LST or 0300 LST during the ascending or descending parts of the orbit, respectively, except poleward of $75^{\circ}$ latitude where the spacecraft shifts from 1500 LST or 0300 LST and vice versa in the opposite polar hemisphere. Note that the actual local true solar time can deviate from the nominal local mean solar time by up to 0.7 Mars hours over the course of the Mars year. In addition, the local mean solar time varied in ranges between 0.2 and 0.5 Mars hours over the duration of the MRO mission.

As noted previously, prior to September 2010 the nominal MCS data-taking mode was in-track, which means that at latitudes equatorward of about $\pm 75^{\circ}$ measurements are only available at 1500 and 0300 LST. However, the cross-track observing mode that was initiated in September 2010 (Kleinböhl et al., 2013) provides measurements at additional LSTs, and data collection is still ongoing. At low latitudes cross-track measurements are offset by $\sim \pm 1.5-2.0$ hours in LST from in-track. This local time difference increases to over 3 hours towards high latitudes, such that analyses that rely on this local time difference, e.g. the extraction of semidiurnal tide parameters, are most robust at high latitudes. As shown by Kleinböhl et al. (2013), the LST spacing afforded by this observational mode is adequate to extract the semidiurnal tide, which these authors did for the solar asynchronous component. Wu et al. (2015) additionally extracted diurnal and semidiurnal solar-asynchronous tides from MCS temperature measurements made in this operational mode. Since 12-14 longitudes are sampled in a given day, diurnal and semidiurnal tides with zonal wavenumbers as large as 6 (both eastward and westward) are in principle capable of being extracted from these data.

For the present study, tidal specifications are sought at $76 \mathrm{~km}$ since the expectation is that all tidal components capable of growing exponentially to $100 \mathrm{~km}$ and beyond would have their maximum amplitudes in the MCS data set at this altitude. (Although MCS data often extend beyond $80 \mathrm{~km}$, utilizing data at this altitude would introduce additional gaps.) All available MCS temperature data at $76 \mathrm{~km}$ between Sept 2010 - Jul 2019 are utilized except for the global dust storm period of Jun - Aug 2018, since tides 
are anomalous during global dust storms, and not part of normal climatology. Other dusty periods are included since they are arguably part of climatology.

\subsection{Tidal fitting}

In our experimentation with these data, we found that aliasing between diurnal and semidiurnal components could occur. The robustness of tidal fits is likely affected by variability associated with uncertainties in the temperature measurements at $76 \mathrm{~km}$. The following steps were therefore taken to ameliorate these issues. To improve statistics, all available MCS data between Sept 2010 - Jul 2019 are binned and averaged with respect to latitude, longitude, LST and Ls. Multi-year averaging is a reasonable approach since prior studies have noted the repeatability of salient structural features in MCS tides from year to year (Moudden and Forbes, 2015; Wu et al., 2015). Similar multi-year averaging to delineate tides has been successfully applied at Earth. Also, we found that fitting across zonal wavenumbers $(s-n)=-6$ to +6 to diurnal and semidiurnal components separately, instead of together, eliminated aliasing between the two while yielding the nearly the same diurnal-mean temperatures.

Typical portrayals of data distributions and fitting are provided in Figure 1. The distribution of data points in latitude-LST space is shown in Figure 1(a), which is typical of other Ls periods. The distribution of points poleward of $\pm 60^{\circ}$ latitude is rather good. However, local time coverage equatorward of $\pm 40^{\circ}$ is potentially of concern for extracting semidiurnal tidal components, particularly SW2 which can alias with the zonaland diurnal-mean temperature; at a fixed local time, both SW2 and the zonal mean temperature are longitude-independent (cf. Equation (2)). In addition, potential systematic uncertainties between in-track and cross-track observations can introduce errors in the amplitude of SW2 as well as the zonal mean temperature when tidal modes are fit over a large local time gap. The local mean solar time of MCS observations varied in ranges between 0.2 and 0.5 Mars hours over the duration of the MRO mission; also the actual local true solar time can deviate from the nominal local mean solar time by up to 0.7 Mars hours over the course of the Mars year (see Figure S1 in the Supporting Information File). Hence using multi-year averages of the data reduces random errors, assists with local time coverage at low latitudes, and also allows to have some data bins being not exclusively populated by in-track or cross-track measurements, which somewhat mitigates possible systematic uncertainties between these observation types. To deal with 
the disparity between the numbers of data points in some bins versus others, we weighted the bin-averaged data points according to the cube root of the number of data points. In practice this results in little overall difference with results in which all bin-averaged data points were weighted equally. This insensitivity serves as a secondary validation of the robustness of the tidal determinations.

As shown in Section 4, there is often a remarkable correspondence between MCD and MCS seasonal-latitudinal structures and amplitudes that serves as a kind of mutual validation of the MCS tidal analysis on one hand and the MCD model results on the other. As demonstrated there, for a given space-based zonal wavenumber, the corresponding diurnal and semidiurnal tides have markedly different latitude structures, which is further evidence that aliasing between them does not exist to a measurable degree. However, for the reasons noted above, the extraction of the solar synchronous semidiurnal tide (SW2) is still problematic as it is potentially aliased with the zonal mean. Therefore, the seasonal-latitudinal variability of SW2 derived from MCS and depicted in this paper must be viewed with this caution in mind.

Figures 1(b)-1(f) show some typical examples of the fits to MCS temperatures (residuals from zonal mean) with respect to LST at a few latitudes, Mars longitudes and Ls.

Each panel illustrates the fitted data points with their attendant standard deviations, the semidiurnal fits to these data points subject to the above weighting, the diurnal fits, and the combined diurnal and semidiurnal fits. The fits enable latitude versus Ls depictions of amplitudes and phases of each tidal component to be constructed, which are performed within windows of length $30^{\circ}$ Ls moved forward in increments of $15^{\circ}$ Ls. The largest amplitude tides consist of DW1, SW2, DE3, DE2, DE1, SE1, S0, and SW1. In Section 4, the latitude versus Ls depiction of each of these tides at $76 \mathrm{~km}$ is compared with that from the MCD, and the MCD height-latitude structure of each tide and its vertical coupling characteristics are addressed. First, however, a brief description of the MCD is provided in the next subsection.

\subsection{MCD model description}

207

The Mars Climate Database (MCD) provides meteorological fields that are derived from a Mars general circulation model (MGCM) developed at the Laboratoire de Météorologie Dynamique (http://www-mars.lmd.jussieu.fr). The LMD-MGCM is rooted in the early 
work of Forget et al. (1999), but since then has adopted more sophisticated and realistic treatments of dust and water cycles, photochemistry, radiative transfer, and solar radiative fluxes. Particularly relevant to the current work, the modern version extends into the thermosphere and ionosphere Gonzalo-Galindo et al. (2009, 2013, 2015). In addition to simulations pertaining to individual Martian Years (MY 24-33), the MCD currently offers users eight baseline database scenarios related to dust and solar EUV conditions. In this study, we use the climatology scenario from MCD Version 5.3 (Millour et al., 2018), in which dust distributions are based on those observed in Mars years when no global dust storms occurred, and average solar EUV conditions are assumed. The MCD outputs are provided in increments of $30^{\circ} \mathrm{Ls}$, which are interpolated to $15^{\circ}$ to be consistent with the fitting window of the MCS observations.

\subsection{Brief review of tidal theory}

Following the terrestrial example set by Truskowski et al. (2014), in this paper we will find it instructive to employ some aspects of classical tidal theory to interpret the height-latitude structures and propagation characteristics of tidal components in Mars' atmosphere. Classical tidal theory refers to solutions of the momentum, thermal energy, continuity and state equations of the atmosphere that are linearized relative to a horizontallystratified basic state without dissipation (e.g., Chapman and Lindzen, 1970). The early development of tidal theory with specific application to Mars is attributable to Zurek (1976). Assuming dependent variables such as horizontal and vertical winds, temperature, density and pressure or geopotential to be periodic in $t$ and $\lambda$ as in (1) eliminates derivatives with respect to $t$ and $\lambda$. This permits consolidation of the problem into a single second-order partial differential equation for the perturbation geopotential, and other variables to be expressed in terms of the geopotential. This equation is separable in $z$ and $\theta$, leading to an eigenfunction-eigenvalue problem where the $\theta$ dependence is embodied in Laplace's tidal equation and the height dependence is embodied in a "vertical structure equation" which also contains specification of the thermal source.

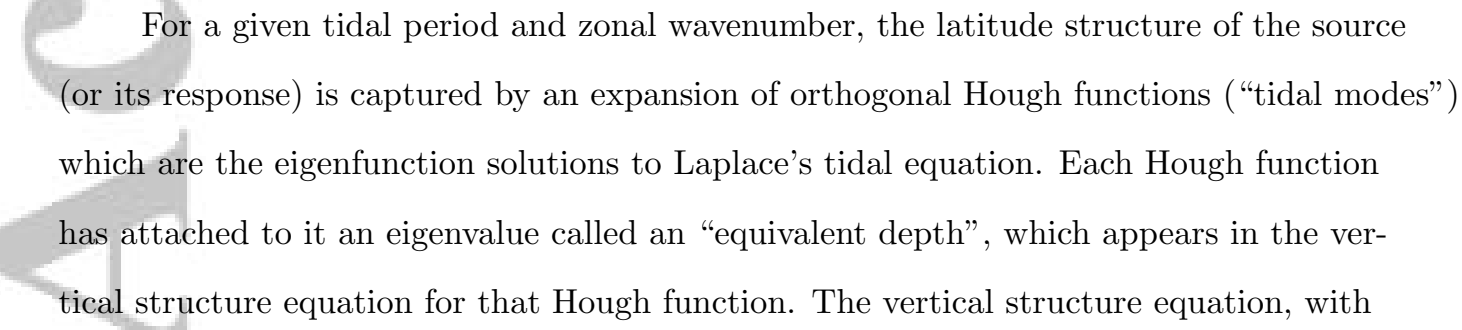


the same form as a 1-dimensional time-independent Shrodinger equation, can be solved analytically in the case of an isothermal background state, or numerically in the case of a more general vertical distribution of temperature. In this context an approximate vertical wavelength can be derived as follows:

$$
\lambda_{z} \approx \frac{2 \pi H_{0}}{\sqrt{\frac{H_{0}}{h_{n}}\left(\kappa+\frac{d H}{d z}\right)-\frac{1}{4}}}
$$

where $\kappa=\frac{R}{c_{p}}=.223$ at Mars, $R$ is the gas constant and $c_{p}$ is the specific heat at constant pressure, $H_{0} \approx 8.5 \mathrm{~km}$ is the mean scale height and $\frac{d H}{d z} \approx-0.046$ below $100 \mathrm{~km}$ at Mars. These values are consistent with those computed for $\mathrm{Ls}=180^{\circ}$ zonal-mean values from MCD v5.3, and those reflected in Viking 1, Viking 2, Curiosity and Opportunity Lander profiles. Given the approximate nature of classical tidal theory in the context of interpreting a GCM, nothing more sophisticated than this approach is considered useful. Thus, a single approximate vertical wavelength can be assigned to each tidal mode at Mars, applicable below $100 \mathrm{~km}$. Note also that for $h_{n}>6.0$, the quantity under the square root is negative, yielding an imaginary $\lambda_{z}$, thus implying a non-propagating (evanescent) solution. This occurs for very large vertical wavelengths. Classical tidal theory also admits negative equivalent depths for diurnal tides (Chapman and Lindzen, 1970; Lindzen, 1966; Zurek, 1976) which also correspond to evanescent solutions, and which capture diurnal responses at mainly middle-high latitudes. See further discussion on this point in subsection 3.1 .

The first two to four Hough functions for each of the tidal components considered in this study can be found in Figure S2 of the Supporting Information file, and some are illustrated explicitly within several of the figures to follow. The vertical wavelengths corresponding to these tidal modes according to (3) are tabulated in Table 1, which are only intended to provide rough estimates. In addition to $h_{n}>6.0$, Hough modes with $h_{n}<$ $0.44\left(\lambda_{z}<30 \mathrm{~km}\right)$ are indicated by symbols in Table 1 . The former do not propagate vertically while it was determined by viewing MCD tidal structures that the latter do not effectively propagate above about $100 \mathrm{~km}$ due to their susceptibility to dissipation. The remaining tidal modes in Table 1 are those that most effectively couple the lower and upper atmospheres of Mars, although the absolute effectiveness of any given mode also depends on the magnitude of its forcing. In the following, we will use classical tidal theory as a starting point for interpretation of the MCD height-latitude structures, and 
then considering dissipation and Doppler-shifting effects by the mean wind field as needed to provide qualitative but more realistic interpretations.

In addition to neglecting mean wind effects and dissipation, classical tidal theory also neglects the nonlinear terms in the momentum and thermal energy equations. As shown for Earth (Truskowski et al., 2014) and Mars (Moudden and Forbes, 2008a), tidetide and tide-SPW interactions can yield tides with different periods and/or zonal wavenumbers than the original interacting waves. This follows from Teitelbaum and Vial (1991) who more generally show that the nonlinear interaction between two primary waves with [frequency, zonal wavenumber $]=\left[\sigma_{1}, s_{1}\right]$ and $\left[\sigma_{2}, s_{2}\right]$ gives rise to two secondary waves (SW) with the "sum" and "difference" frequencies and zonal wavenumbers of the primary waves: $S W^{+}=\left[\sigma_{1}+\sigma_{2}, s_{1}+s_{2}\right]$ and $S W^{-}=\left[\sigma_{1}-\sigma_{2}, s_{1}-s_{2}\right]$. The two interacting waves can be any combination of tides, traveling planetary waves, or SPWs (for which the frequency is zero). For example, consider the interaction between SPW1 ([0.0d $d^{-1}$, $+1])$ and SW2 $\left(\left[2.0 d^{-1},+2\right]\right)$ :

$$
S P W 1 \times S W 2 \rightarrow S W 3+S W 1
$$

where SW3 $=\left[2.0 d^{-1},+3\right]$ and SW1 $=\left[2.0 d^{-1},+1\right]$; or the interaction between DW1 $\left(\left[1.0 d^{-1},+1\right]\right)$ and DE2 $\left(\left[1.0 d^{-1},-2\right]\right)$ :

$$
D W 1 \times D E 2 \rightarrow S E 1+S P W 3
$$

where SE1 $\left.=\left[2.0 d^{-1},-1\right]\right)$ and SPW3 $=\left[0.0 d^{-1},+3\right]$

Although Teitelbaum and Vial (1991) suggest that self-interactions are not likely to be efficient, there are indications in the literature that this is not necessarily true for propagating waves if the background wind conditions are favorable (e.g., Pogoreltsev, 2001). This appears to be the case for the 6 -hour $s=+4$ solar-synchronous tide generated by SW2 self-interaction $(S W 2 \times S W 2)$, which achieves meridional wind amplitudes up to $27 \mathrm{~ms}^{-1}$ at $130 \mathrm{~km}$ at $20^{\circ} \mathrm{S}$ in the terrestrial calculations of Angelats i Coll and Forbes (2002). As shown in the simulations of Huang et al. (2007), a SW2 secondary-wave temperature amplitude of $18 \mathrm{~K}$ at $106 \mathrm{~km}$ over the terrestrial equator can result from the DW1 self interaction $(D W 1 \times D W 1)$. 


\section{Results}

In this section, comparisons are performed between temperature amplitudes of the DW1, SW2, DE3, DE2, DE1, SE1, S0, and SW1 tidal components at $76 \mathrm{~km}$ from the MCD and those derived from MCS observations at $76 \mathrm{~km}$ altitude by the methodology described in Section 2. These tidal components all achieve amplitudes greater than 2.0 $\mathrm{K}$ in both MCS and MCD at $76 \mathrm{~km}$. In addition, given the reasonable agreement between MCD and MCS tides at $76 \mathrm{~km}$ in many respects, we also explore the vertical propagation of these tides well into the thermosphere, and interpret their height-latitude structures in terms of dissipative processes and background propagation conditions. Due to their relevance to the interpretation of longitude structures measured by, e.g., MGS, MO, MRO and MAVEN, the solar-asynchronous tidal components are furthermore considered in terms of the total mass density perturbations that they produce.

The largest tidal components in both the MCD and MCS observations are the solarsynchronous components, DW1 and SW2. These are considered in the following subsection 3.1. As noted in Section 1 in connection with (2), solar-synchronous tides are longitudeindependent when viewed from a quasi-solar-synchronous satellite perspective. The diurnal and semidiurnal solar-asynchronous tides, which do determine the longitude structures measured from such satellites as MGS, MO, MRO and MAVEN, are considered in subsections 3.2 and 3.3, respectively. The MCD demonstrates that SPW can sometimes produce measurable longitude variations above $100 \mathrm{~km}$, and these are discussed briefly in subsection 3.4. Subsection 3.5 deals more specifically with tidal density perturbations due to tides at $108 \mathrm{~km}$ and $172 \mathrm{~km}$, heights relevant to interpretation of MGS, MO, MRO and MAVEN respectively. A comparison between MCD longitude structures and those measured by MGS is also performed and assessed in subsection 3.5.

\subsection{Solar-synchronous ("migrating") diurnal and semidiurnal tides}

Figures 2(a)-2(d) illustrate comparisons between MCS and MCD latitude versus Ls amplitude structures at $76 \mathrm{~km}$ for the diurnal and semidiurnal solar-synchronous tides, DW1 and SW2. The variability in these structures represent some combination of variability due to sources in the lower atmosphere, and propagation conditions between the sources and $76 \mathrm{~km}$ altitude. For DW1 (Figures 2(a) and 2(c)), MCS and MCD share the common feature of maxima in the equatorial-region and at higher latitudes in both hemi- 
spheres. The equatorial DW1 maxima occur around the equinoxes, Ls $=330-030^{\circ}$ and $\mathrm{Ls}=150-210^{\circ}$, in both the MCS and MCD analyses. The non-equatorial maxima occur around $\pm 75^{\circ}$ latitude. For MCS the non-equatorial maxima in one hemisphere are not always accompanied by the counterpart in the opposite hemisphere (i.e., Ls $=210$ $300^{\circ}$ in the S. Hemisphere). The presence(absence) of DW1 in the MCS data(MCD) between about $\mathrm{Ls}=30-140^{\circ}$ corresponds exactly to the radiatively-active "aphelion cloud belt", which contributes substantial heating (Wilson, 2014; Wilson and Guzewich, 2014) and appears to be underestimated in MCD V5.3 (Navarro et al., 2014). In addition, when MCS non-equatorial maxima do exist, they are sometimes not spread towards the poles to the degree that they are in the MCD (i.e., Ls $=0-240^{\circ}$ in the N. Hemisphere and Ls $=300-360^{\circ}$ in both hemispheres). Finally, it is noted that MCD amplitudes generally achieve $\sim 50 \%$ higher amplitudes than those obtained from MCS.

For SW2, the MCS (Figure 2(b)) and MCD (Figure 2(d)) amplitudes also tend to maximize at middle latitudes, with more of a tendency to minimize at equatorial latitudes than DW1. MCD reveals modest-amplitude ( 4-5 K) equatorial maxima around $\mathrm{Ls}=190^{\circ}$ and $\mathrm{Ls}=350^{\circ}$, with an MCS equatorial maximum $(\sim 7 \mathrm{~K})$ occurring around $\mathrm{Ls}=320^{\circ}$. MCS SW2 maximum amplitudes generally exceed those in MCD by $\sim 50 \%$.

However, as emphasized in subsection 2.2, the MCS SW2 amplitudes and seasonal-latitudinal structures must be viewed with some caution, since some aliasing with the zonal mean can potentially still be included within them.

Figure 2(e) illustrates the latitude versus Ls structure of the zonal- and diurnalmean zonal wind (hereafter zmU or $\bar{U}$ ) at $76 \mathrm{~km}$. At this point it should be noted that the $\bar{U}$ distribution in Mars atmosphere is not well-known, and other models that include more extensive treatment of parameterized gravity waves (e.g., Gilli et al., 2020; Medvedev et al., 2011) exhibit significant differences from MCD v5.3 while also sharing many salient features. The MCD $\bar{U}$ distribution at this altitude and depicted in Figure 2(e) is mainly characterized by westward winds equatorward of $\sim \pm 40^{\circ}$ latitude, and eastward winds at higher latitudes. This provides an initial hint that $\bar{U}$ may be playing a role in determining some aspects of the DW1 and SW2 structures common to MCS and MCD, since westward-propagating waves preferentially propagate into eastward wind regimes where they are Doppler-shifted to higher frequencies and hence are less susceptible to dissipation. Of course, the effects of $\bar{U}$ are to impose a seasonal-latitudinal modulation on top of the variability due to the sources of DW1 and SW2, so that establishing cause-effect 
relationships is not completely straightforward. Figure $2(\mathrm{f})$ shows that the middle- to high-latitude maxima in the MCD SW2 amplitude distribution also persists to $172 \mathrm{~km}$ altitude, but we will find below that this is not true for DW1. In Figures 3 and 4 and discussion below, it is our aim to clarify the role of $\bar{U}$ on DW1 and SW2 by examining their height-latitude structures along with that of $\bar{U}$ and our knowledge of relevant dissipative processes.

Figures 3(a) and 3(b) illustrate the height-latitude structure of DW1 temperature amplitudes and phases, respectively, from MCD for $\mathrm{Ls}=150^{\circ}-180^{\circ}$. The overall structure in the middle atmosphere resembles the DW1 structure based on MCS data (Lee et al. 2009). The broad high-amplitude signature above $\sim 130 \mathrm{~km}$ with no phase progression with height is the response to in-situ EUV solar radiation absorption and heating. Similarly the broad temperature response below $10-20 \mathrm{~km}$ is the result of near-surface heating. The 3 maxima below $100 \mathrm{~km}$ in Figure 3(a) are signatures of the tide that is excited near the surface and propagates to higher altitudes, as indicated by their downward phase progressions with height in Figure 3(b). The equatorial-region maximum propagates upward with a vertical wavelength of order $50 \mathrm{~km}$ between 20 and $70 \mathrm{~km}$, and which shortens abruptly around $105 \mathrm{~km}$. Meanwhile the phases associated with the mid- to highlatitude maxima reflect downward phase progression with height (indicating upward propagation) between 20 and $70 \mathrm{~km}$, but nearly evanescent phase behavior above about 70 $\mathrm{km}$. Note also that the mid- to high-latitude maxima are out of phase with the maximum between $\pm 30^{\circ}$ latitude.

Based on our knowledge of classical tidal theory, which neglects the effects of mean winds and dissipation, the near-surface heating will excite a combination of evanescent and propagating orthogonal modes or "Hough functions" that are solutions to Laplace's tidal equation. The evanescent modes predominate at middle to high latitudes and remain trapped near the surface, whereas the propagating modes are confined to low latitudes (Chapman and Lindzen, 1970; Zurek, 1976). The first symmetric propagating mode propagates upward as the primary contributor to the response, since higher-order propagating modes have relatively short vertical wavelengths with high susceptibility to dissipation. From Table 1 the vertical wavelength of the first symmetric propagating mode is about $32 \mathrm{~km}$ according to classical tidal theory, and has the latitude structure given in Figure 3(c). The horizontal structures and vertical wavelengths quoted in connection with Figures 3(a) and 3(b) differ significantly from these expectations, and from obser- 
vations and modeling of its terrestrial counterpart (see, e.g., Figure A1 in Forbes et al., 2018). Therefore, some effort is expended below to explain the nature of DW1 at Mars.

In connection with the terrestrial atmosphere, Forbes and Vincent (1989) developed simple analytic extensions to classical tidal theory that elucidated the effects of mean winds and dissipation. They found that in regions of eastward(westward) $\bar{U}$, Dopplershift of the diurnal tide to higher(lower) frequencies resulted in increased(decreased) vertical wavelengths and reduced(enhanced) susceptibility to dissipation. In this context, consider the latitude-height distribution of $\bar{U}$ in Figure 3(d), which is characterized by a westward jet of order $120 \mathrm{~ms}^{-1}$ above $90 \mathrm{~km}$, and eastward jets of order $75-100 \mathrm{~ms}^{-1}$ extending from roughly $30 \mathrm{~km}$ to $100 \mathrm{~km}$ at middle to high latitudes in each hemisphere. At least qualitatively, the Doppler-shifting effects predicted by Forbes and Vincent (1989) suggest why the vertical wavelengths in Figure 3(b) change the way they do, and why in Figure 3(a) vertical propagation of the equatorial maximum is apparently impeded by the westward jet, and the non-equatorial maxima follow the eastward jets.

Forbes and Vincent (1989) also showed that the non-equatorial maxima in Figure 3 (c) shifted poleward(equatorward) in the presence of eastward(westward) $\bar{U}$, and amplified(diminished) in amplitude. However, they did not discuss this effect further, and in their example used rather modest Doppler shifts in comparison to the conditions represented in Figure 3(d). The confinement of vertically-propagating DW1 components to low latitudes (c.f. Figure 3(c)) and the dominance of vertically-trapped solutions at middle to high latitudes is related to the ratio of the frequency of DW1 to the planetary rotation rate, by analogy with the behavior of internal gravity waves in a rotating planar fluid (Lindzen, 1971; Chapman and Lindzen, 1970). Vertical propagation of gravity waves in a rotating planar system requires the gravity wave frequency $\sigma$ to be less than the BruntVäisälä frequency and greater than twice the rotation rate $(\Omega)$, or $\sigma>2 \Omega$. On a rotating sphere the vertical component of the rotation vector, $\Omega \sin \theta$ (where $\theta=$ latitude), replaces $\Omega$, so that the requirement for vertical propagation becomes $\sigma>2 \Omega \sin \theta$. For DW1 $(\sigma=\Omega)$, this requirement is only met equatorward of $30^{\circ}$ latitude, which is consistent with low-latitude confinement of DW1 in Figure 3(c).

Now consider replacing $\sigma$ by a Doppler-shifted frequency, $\sigma_{D}=\Omega+s \bar{U} / a \cos \theta$ where the zonal wavenumber $s=1$ for DW1. As $\sigma_{D}$ increases, then the latitude at which the transition from propagating to evanescent solution should increase with latitude. Put 
another way, one can ask what the Doppler-shifted frequency (or $\bar{U}$ ) must be at a given

latitude for the solution to remain propagating:

$$
\begin{array}{r}
\sigma_{D}>2 \Omega \sin \theta \\
\Omega+\bar{U} / a \cos \theta>2 \Omega \sin \theta \\
\bar{U}>241 \cos \theta(2 \sin \theta-1) m s^{-1}
\end{array}
$$

where $241 \cos \theta \mathrm{ms}^{-1}$ is the zonal phase speed of DW1 and $a$ is the planetary radius. Note that $\cos \theta=0$ at $\theta=90^{\circ} ;(2 \sin \theta-1)=0$ at $\theta=30^{\circ}$; and $(2 \sin \theta-1)<0$ equatorward of $30^{\circ}$ latitude. Therefore, for DW1 propagating solutions to exist at (for example) $45^{\circ}, 60^{\circ}$ and $75^{\circ}$ latitude, then $\bar{U}$ must exceed 70,88 , and $59 \mathrm{~ms}^{-1}$, respectively. Comparing these values with those in Figure 3(d), these conditions are met in the S. Hemisphere, and nearly met in the N. Hemisphere, lending credibility to the argument that the extension of DW1 maxima to high latitudes as in Figure 3(a) can be explained as a Doppler-shift effect. At Earth, the zonal phase speed of DW1 is $463 \cos \theta \mathrm{ms}^{-1}$, mainly due to differences in the radii of the planets (6370 km for Earth; $3397 \mathrm{~km}$ for Mars). This, combined with the much larger zonal wind speeds at Mars, accounts for the differences in the latitudinal structures of DW1 at the two planets. This contrast between mean wind effects at Mars and Earth was also recognized by Takahashi et al. (2006), whose work we now discuss in the context of DW1.

Mars GCM results presented by Takahashi et al. (2006) also exhibited an increase in DW1 vertical wavelength to about $50 \mathrm{~km}$ compared to classical tidal theory, and an extended DW1 temperature response peaking near $\pm 60^{\circ}$ latitude between 60 and $80 \mathrm{~km}$. The corresponding $\bar{U}$ distribution was symmetric about the equator, with eastward winds similar to those in the N. Hemisphere in Figure 3(d), and maximum westward winds of order $20 \mathrm{~ms}^{-1}$ below $80 \mathrm{~km}$ at the equator. Using a linear tidal model, they performed numerical experiments that showed that the eastward winds at low latitudes were insufficient to produce the observed increase in vertical wavelength through Doppler shifting, and that instead that it was the term in the zonal momentum equation that included the meridional shear of $\bar{U}$ (i.e., the zonal-mean vorticity, $\bar{\zeta}$ ) that was responsible for this effect. The zonal-mean vorticity only appears with the Coriolis parameter $f$ in the zonal momentum equation (Andrews et al., 1987), $(f+\bar{\zeta})$, and so does not really translate to a change in the planetary rotation rate. 
The importance of $\bar{\zeta}$ to DW1 was first noted by McLandress (2002) who demonstrated its contribution to the low-latitude seasonal variability of DW1 in Earth's middle atmosphere. Neither Takahashi et al. (2006) or McLandress (2002) addresses extreme movement of the DW1 wind or temperature maxima towards the poles. This aspect of the problem is complicated in the Takahashi et al. results, which includes large enough dissipation (parameterized by Rayleigh friction) to couple into trapped modes (see their Figure 4) above about $70 \mathrm{~km}$, which significantly spreads the temperature structure towards the poles (see their Figure 3(a)). Therefore the role of $\bar{\zeta}$ in producing these highlatitude maxima is obscured by this effect. The LMD GCM does not include any prescribed eddy dissipation in the momentum equations, and therefore the spreading to higher latitudes in the MCD output must be a mean wind effect. Although the GCM includes radiative damping in the thermal energy equation, this does not render the tidal equations inseparable, and thus does not induce any mode coupling.

Consider now the discussion at the beginning of this Section concerning the similarities and differences between the latitude-Ls structures of DW1 in MCS data (Figure 2(a)) and in the MCD (Figure 2(c)). In particular, the non-equatorial maxima associated with the MCS DW1 latitude structures often do not extend as far poleward as those in the MCD. The above analysis in terms of Doppler shifting effects now seems to suggest that these disparities may be connected with differences in the intensity and/or height-latitude structure of the zonal-mean eastward jets between the MCD and climatological conditions at Mars. It is also possible that effects associated with vorticity of the background state could also be playing a role, but these are not easily separated from Doppler shifting effects within a GCM framework, and would require linear-model numerical experiments similar to those performed by Takahashi et al. (2006) to disentangle them.

The presence of a westward jet above $90 \mathrm{~km}$ is likely not the only impediment to vertical penetration of DW1 above $100 \mathrm{~km}$, and in fact this westward jet may exist in part to the the deposition of westward momentum by the dissipating DW1. $\mathrm{CO}_{2}$ cooling can serve to damp DW1 temperature perturbations between 100-140 km. This is also the height region where the importance of molecular dissipation, which varies according to $1 / \rho_{0} \sigma \lambda_{z}^{2}$ where $\lambda_{z}$ is the vertical wavelength and $\rho_{0}$ is the background density, begins to significantly damp atmospheric tides. It has also been shown that nonlinear selfinteraction of the terrestrial propagating DW1 tide can diminish its equatorial-region tem- 
perature amplitudes and enhance amplitudes of its non-equatorial maxima compared to a linear solution (Huang et al., 2007), a feature that is consistent with the relative magnitudes seen in Figures 3(a) and 3(c). There is also evidence that the diurnal propagating tide could undergo convective adjustment if the temperature amplitudes become sufficiently large (Lindzen, 1968; Wu et al., 1968; Zurek, 1976). From Figure 3(a) it is clear that the vertically propagating DW1 undergoes abrupt dissipation in the 80-120 km height region. However, sorting out the relative importances of these effects is not a straightforward task within the confines of a GCM, and would benefit from numerical experiments using a simpler model.

Figures 4(a) and 4(c) illustrate examples of MCD SW2 height-latitude structures for $\mathrm{Ls}=60-90^{\circ}$ and $\mathrm{Ls}=300-330^{\circ}$, respectively. The structure in the polar middle atmosphere resembles the SW2 structure derived previously from MCS data (Kleinböhl et al., 2013). Note that $\operatorname{SW} 2(\sigma=2 \Omega)$ satisfies the condition $\sigma>2 \Omega \sin \theta$ all the way to the poles, and thus in the absence of mean winds all SW2 modes are vertically-propagating. Contrary to DW1 where a single tidal mode dominates, for SW2 the first four Hough modes span vertical wavelengths of $47-358 \mathrm{~km}$ based on classical tidal theory where it is assumed that $\bar{U}=0$. These Hough modes consist of the first two symmetric and first two antisymmetric modes, and have sufficiently long vertical wavelengths to enable propagation into the thermosphere. (The horizontal structures of these Hough modes are depicted in the Supporting Information File.) In the presence of the wintertime eastward jets depicted in Figures 4(b) and 4(d), the horizontal structures of these modes will spread to higher latitudes, analogous to that illustrated for DW1. Vertical wavelengths will also lengthen, perhaps admitting even higher-order modes to those capable of propagating into the thermosphere, although use of the term "modes" may be questioned since they are likely no longer orthogonal. What all of this means is that the superposition of these Doppler-shifted latitudinal structures will be able to capture much more complex horizontal and vertical structures than was the case for DW1.

Figure 4(a) is an example of such a complex structure. Note that the winter-hemisphere eastward jet approaches $+200 \mathrm{~ms}^{-1}$ and extends well into the thermosphere. It is flanked by the polar boundary and a westward jet extending from about $30^{\circ} \mathrm{S}$ to $60^{\circ} \mathrm{N}$, with wind speeds approaching $-150 \mathrm{~ms}^{-1}$. This configuration essentially forms a propagation channel for SW2, which extends well into the thermosphere where molecular dissipation limits its growth with height around $150 \mathrm{~km}$. At Ls $=300-330^{\circ}$ (Figures $4(\mathrm{c})$ and $4(\mathrm{~d})$ ) the 
winter hemisphere eastward jet does not exhibit extension into the thermosphere, and a propagation path does not exist to the extent that it does during $\mathrm{Ls}=60-90^{\circ}$. The modest SW2 amplitudes above about $100 \mathrm{~km}$ and between about $\pm 30^{\circ}$ latitude, with larger values prevailing around perihelion, likely originate from heating due to in-situ absorption of EUV solar radiation, by analogy with the thermosphere maximum seen in Figure $3(\mathrm{a})$.

\subsection{Solar-asynchronous ("non-migrating") diurnal tides}

The focus of this subsection is on the solar-asynchronous diurnal tides with MCS temperature amplitudes exceeding $2 \mathrm{~K}$ at $76 \mathrm{~km}$, and these consist of DE3, DE2 and DE1, as illustrated in Figure 5. Moudden and Forbes (2008a) performed numerical experiments with a Mars GCM (however, only for Ls $=75^{\circ}$ ) to better understand the sources of solarasynchronous tides. They concluded that the most important source of DE2(DE1) was the interaction of $m=3(m=2)$ topography with the solar-synchronous diurnal component (DW1) of near-surface solar heating, analogous to the interaction between SPW3(SPW2) and DW1: $S P W 3(S P W 2) \times D W 1 \rightarrow D E 2(D E 1)+D W 4(D W 3)$. The $m=2$ topographyDW1 heating interaction that produces DE1 was in fact first anticipated by Zurek (1976) and its associated temperature response was detected in Mariner 9 IRIS data by Conrath (1976). From Table 2, DE2 and DE1 possess vertical wavelengths in the range corresponding to efficient vertical coupling, whereas DW4 and DW3 consist of short vertical wavelength modes and do not contribute to vertical coupling. Moudden and Forbes (2008a) also conclude the following to be a secondary source of DE2: $S P W 1 \times D E 1 \rightarrow$ $D 0+D E 2$, and that zonal variations in surface thermal inertia and albedo served as secondary contributors to DE1. These authors did not mention DE3, but it seems logical to conclude that it arises from the same DW1 solar heating-topography interaction as DE2 and DE1, except this time with $m=4$.

The general characteristics of DE3, DE2 and DE1 were comprehensively delineated using MGS Thermal Emission Spectrometer (TES) temperature measurements near 25 km by Wilson (2000). It is immediately apparent from Figure 5 that DE3, DE2, and DE1 are confined to latitudes equatorward of $\pm 40^{\circ}$ latitude, have comparable amplitudes with those of MCD, and that the MCS structures tend to be shifted poleward in the N. Hemisphere in comparison to MCD. These tides are sometimes referred to as diurnal Kelvin waves (specifically DK3, DK2, DK1; Wilson, 2000), although strictly speaking only the 
first symmetric Hough mode of a given zonal wavenumber is a Kelvin wave; latitudinal asymmetries and other deviations from Kelvin wave shapes (such as the shifting in latitude of an otherwise Gaussian-like latitude structure), are captured by higher-order Hough modes. In fact, we have determined that all the displayed structures in Figure 5 are well captured by the first 4 Hough modes (see Supporting Information File), the first 3 of which are illustrated in the Figure 6. However, the reconstructed structures (not shown) based on these Hough functions are slightly more confined (by about $10^{\circ}$ in each hemisphere), compared to those displayed in Figure 5. This is due to the Doppler-shifting effect of mean winds, which are now discussed in connection with with DE3, DE2 and DE1 in the context of their vertical propagation characteristics as displayed in Figure 7.

The MCD height-latitude structures of DE3, DE2, and DE1 are shown in Figure $7(\mathrm{a}),(\mathrm{b})$ and (c), respectively, for Ls $=60-90^{\circ}$, along with the corresponding $\bar{U}$ in Figure 7(d). In Figure 7 as well as upcoming Figures 9-11, the plotted variable is percent total mass density perturbation relative to the zonal- and diurnal-mean. Total mass density is the quantity plotted due to the relevance of these tides to longitude structures in density that are evident in MGS, MO, and MRO accelerometer measurements and MAVEN mass spectrometer measurements. The displayed latitude structures are increasingly broad for DE3, DE2 and DE1, consistent with expectations based on classical tidal theory as illustrated in Figure 6. In terms of mean wind effects, propagation of these eastwardpropagating tidal components favors the propagation channel produced by the prevailing westward wind regime that is shifted slightly into the N. Hemisphere.

In the following, we refer to the symmetric and antisymmetric components of DE3, DE2, and DE1 about the equator, and also the phases of these tidal components. These plots are included in Figures S3, S4 and S5, respectively, of the Supporting Information file.

The vertical wavelengths for DE3 in Table 1, and diagnosis of the symmetric and antisymmetric structures of DE3 about the equator (not shown, refer to Figure S2), reveal a few insights relevant to the interpretation of Figure 7(a). The vertical wavelength of the symmetric part is about $80 \mathrm{~km}$ below $100 \mathrm{~km}$, consistent with the value of $64 \mathrm{~km}$ listed in Table 1 and some lengthening due to Doppler-shifting effects of the prevailing westward winds. The vertical wavelength lengthens much further beginning at about 120 $\mathrm{km}$ where dissipation begins to dominate, and the symmetric part of DE3 reaches its max- 
imum value of $12 \%$ at the equator. The antisymmetric part of DE3 also peaks near 120 $\mathrm{km}$ but at amplitudes of $6 \%$ near $\pm 30^{\circ}$ latitude, as compared with $\pm 20^{\circ}$ from classical tidal theory (Figure 6), which is consistent with the latitude broadening mentioned above. Its vertical wavelength can be best discerned from examination of Figure 7(a), noting that the structure is shifted to the $\mathrm{S}$. Hemisphere around $70 \mathrm{~km}$, but a maximum shift towards the N. Hemisphere occurs at about $120 \mathrm{~km}$. This yields an effective vertical wavelength of $\sim 100 \mathrm{~km}$, which includes the lengthening effects of both Doppler shifting and dissipation. Thus, both the first symmetric and antisymmetric modes of DE3 play important roles in determining its latitude-height structure.

A similar situation exists with regard to DE2 (Figure 7(b)), but with distinct differences. The symmetric part (Figure S4) also peaks near $120 \mathrm{~km}$, but with a much larger equatorial maximum of $26 \%$ and vertical wavelength below $100 \mathrm{~km}$ of about $107 \mathrm{~km}$. This value for the vertical wavelength suggests that the value of $122 \mathrm{~km}$ in Table 1 may be an overestimate, but as we noted in connection with (3), Table 1 is only intended to serve as a rough guide. The antisymmetric part of DE2 (Figure S4) has maximum amplitudes that increase monotonically with height from $1 \%$ at $50 \mathrm{~km}$ to about $9 \%$ near $170 \mathrm{~km}$. The latitudinal maxima shift from about $\pm 20^{\circ}$ latitude near $50 \mathrm{~km}$, to $\pm 30-50^{\circ}$ near 120 $\mathrm{km}$, to $\pm 20-40^{\circ}$ latitude at $170 \mathrm{~km}$. We interpret this degree of spreading to be consistent with Doppler-shift modification of the first antisymmetric mode for DE2 plotted in Figure 6(b). As with DE3, the influences of the antisymmetric part of DE2 are evident in the total structure plotted in Figure 7(b). The maximum hemispheric shifts occur towards the S. Hemisphere at $80 \mathrm{~km}$, to the N. Hemisphere at $120 \mathrm{~km}$, and back towards the S. Hemisphere $170 \mathrm{~km}$. These yield vertical wavelengths of order $80 \mathrm{~km}$ between 80 -

$120 \mathrm{~km}$ and $100 \mathrm{~km}$ between 120-170 km.

DE1 is particularly interesting since the first symmetric component is a resonant oscillation (or "normal mode (NM)") in Mars' atmosphere (Zurek, 1976, 1988). Indeed, the symmetric part of Figure 7(c) (refer to Figure S5) shows little evidence for phase progression with height, as would be expected for a NM. It achieves a maximum value of $21 \%$ near $120 \mathrm{~km}$. The antisymmetric part of Figure 7(c) (refer to Figure S5) shows evidence of the first antisymmetric Hough mode with maxima near $\pm 30^{\circ}$ latitude, and this accounts for most of the asymmetry seen at altitudes above $150 \mathrm{~km}$ and between 50 and $80 \mathrm{~km}$. However, there is a broad antisymmetric component that extends nearly pole to pole that can only be accounted for by evanescent tidal components which arise through 
"mode coupling" induced by dissipation of DE1 between 100 and $150 \mathrm{~km}$ in the presence of strongly asymmetric mean winds. The presence of these evanescent components accounts for the shift of the maximum at $120 \mathrm{~km}$ into the N. Hemisphere, but the maximum above $100 \mathrm{~km}$ near the S. Pole arises from the nonlinear interaction

$$
S P W 1 \times D 0 \rightarrow D W 1+D E 1
$$

according to the numerical experiments conducted by Moudden and Forbes (2008a). The

MCD D0 shows a temperature(density) maximum of $5-10 \mathrm{~K}(3-6 \%)$ in this region for Ls

$=60-90^{\circ}$ (not shown). The SPW1 counterpart is illustrated in subsection 3.4 (Figure

10(a)). Forbes et al. (2018) interpreted winter hemisphere $s=1 \sim 10-15 \%$ polar-region density perturbations in both $\mathrm{MO}$ and $\mathrm{MRO}$ accelerometer measurements in terms of some combination of D0 and SPW1.

\subsection{Solar-asynchronous ("non-migrating") semidiurnal tides}

The MCS and MCD latitude-Ls structures at $76 \mathrm{~km}$ for the semidiurnal solar-asynchronous tides that exceed $2 \mathrm{~K}$ in amplitude are shown in Figure 8, and consist of SE1, S0 and SW1. Examples of height-latitude structures are also shown in Figure 9 for Ls $=240-270^{\circ}$. For the convenience of interpreting structures in both Figures 8 and 9, a subset of Hough modes for SE1 and S0, and the height-latitude distribution of zonal-mean zonal wind are also provided in Figure 9 . analyses of MCS temperatures (Lee et al., 2009; Guzewich et al., 2012; Moudden and Forbes, 2015; Wu et al., 2015), and Wu et al. (2015) were the first to report the presence of SW1 which they interpreted in terms of $S P W 1 \times S W 2$ nonlinear interaction (i.e., expression (4)). S0 was suggested as a possible contributor to observed longitude wave2 density variability measured by $\mathrm{MRO}$ above $80^{\circ}$ latitude around $\mathrm{Ls}=75-80^{\circ}$ by Forbes et al. (2018); however, it was indistinguishable from DE1 due to the nature of MRO LSTlongitude sampling. From their numerical experiments Moudden and Forbes (2008) concluded that S0 and SE1 were primarily generated by modulation of the SW2 component of solar radiative heating by zonal wavenumber $m=2$ and $m=3$ components of topography, respectively. They also found SW1 in their simulation, but could not distinguish whether this was generated by SW2 solar radiative interaction with $m=1$ topography, or nonlinear interaction between SW2 and SPW1. 
MCS and MCD SE1 temperature amplitudes in Figures 8(a) and 8(b) are distinguished by their 3-peaked structures in latitude (for MCS, only between Ls $=180-360^{\circ}$ ), and their similarities in amplitude. From Table 1, the first symmetric and antisymmetric modes of SE1 are evanescent, and the second symmetric and antisymmetric modes have vertical wavelengths of $134 \mathrm{~km}$ and $74 \mathrm{~km}$, respectively. Therefore, compared to the diurnal tides in the previous subsection, a more structured response is expected. The MCS structures between Ls $=30-150^{\circ}$ require multiple Hough functions (refer to Figure S2) to replicate, including the 4-peaked second antisymmetric mode and first and second symmetric modes. Interestingly, MCD reflects the strong presence of the second antisymmetric mode during this same period, but combines with the second symmetric mode (not shown) to produce a much different structure than MCD. Between Ls $=$ $210-330^{\circ}$ the second symmetric mode is important for both MCD and MCS, giving rise to the 3-peaked structures.

For S0, it is important to note that non-zero temperature amplitudes at the poles are possible, and the fundamental mode is antisymmetric, in contrast to the complete set of non-zonally symmetric tides. The MCS and MCD amplitude distributions of S0 are remarkably similar, and with comparable amplitudes. Between about Ls $=180-360^{\circ}$, MCS and MCD are both dominated by a 4-peaked structure with a null at the equator, whereas between Ls $=0-180^{\circ}$ both model and data consist of 3 peaks but with most of the activity in the N. Hemisphere. As with SE1, the first two Hough modes possess evanescent vertical structures (see Table 1), and the second antisymmetric and symmetric modes have vertical wavelengths from classical tidal theory of $99 \mathrm{~km}$ and $63 \mathrm{~km}$, respectively, thus leading to the expectation that SE1 will consist of a higher-order structured response. Indeed, both MCS and MCD consist in large part of the second antisymmetric mode, with maxima near $\pm 20-30^{\circ}$ and near-polar latitudes, with a null at the equator.

SW1 is somewhat similar to SW2 in that it consists of at least four modes with vertical wavelengths that are capable of efficiently coupling the middle atmosphere with the thermosphere. The means that they can combine in a various ways to produce a variety of complex structures, including an isolated response in one hemisphere. There are several similarities between Figures $8(\mathrm{e})$ and $8(\mathrm{f})$ including the elongated structure between $\mathrm{Ls}=0-240^{\circ}$ in the S. Hemisphere, complementary maxima in the N. Hemisphere between $\mathrm{Ls}=90-210^{\circ}$, the maximum around $\mathrm{Ls}=300^{\circ}$ and $20^{\circ} \mathrm{N}$, and the elongated structure poleward of this maximum between $\mathrm{Ls}=240-330^{\circ}$, albeit at $60^{\circ} \mathrm{N}$ for $\mathrm{MCD}$ 
and about $75^{\circ}$ for MCS. The maximum amplitudes in Figure $8(\mathrm{f})$ are about $40 \%$ lower for MCD than MCS, so it takes a little effort to see these similarities on plots with the same color scales.

\subsection{Stationary planetary waves}

SPWs are generally not thought to be capable of propagating into the thermosphere, primarily based on the lack of reported observations of such penetration at Earth, as well as terrestrial modeling results (Pogoreltsev et al., 2007); perhaps also because early interpretations of SPWs in aerobraking densities (Keating et al., 1998) were shown to be unlikely in favor of a solar-asynchronous tide interpretation (Forbes and Hagan, 2000; Joshi et al., 2000; Wilson, 2002). However, Forbes et al. (2018) recently discussed possible interpretation of winter polar-region $s=1$ features in MO and MRO densities, and suggested a connection with either SPW1 or a displaced polar vortex, as well as D0. SPW can also be generated by nonlinear wave-wave interactions, as exemplified in (5) and demonstrated in numerical simulations by Angelats i Coll et al. (2004) and Moudden and Forbes (2008a). A few examples of SPWs in MCD perturbation densities from the zonal mean are shown in this section.

Height-latitude depictions of MCD SPW1, SPW2, and SPW3 density perturbations are shown in Figures 10(a), (b), and (c). SPW1 is particularly large, approaching $30 \%$ near 100 km. Moudden and Forbes (2008a) also obtained a similar SPW1 structure in their Ls $=75^{\circ}$ simulation, but with maximum amplitudes of about $13 \%$ near $80 \mathrm{~km}$. Upward propagation of SPWs in the prograde jet is well known from previous studies of Mars' lower and middle atmosphere (Hollingsworth and Barnes, 1996; Guzewich et al., 2012). SPW2 in Figure 10(b), with amplitudes of order 5-10\% near $170 \mathrm{~km}$, possesses a similar structure to DE1 (see Figure 7(c)) above $100 \mathrm{~km}$, and it likely arises from

$$
D W 1 \times D E 1 \rightarrow S 0+S P W 2
$$

SPW3 in Figure 10(c), with maximum amplitudes of order 8\%, appears to have its origins in the nonlinear interaction

$$
D W 1 \times D E 2 \rightarrow S E 1+S P W 3
$$

This is consistent with the coincidence of the large amplitudes of DE2 in Figure 7(b) with those of the in-situ forced DW1, which is prevalent in the thermosphere. 
Figures 10(d), (e) and (f) illustrate the latitude versus Ls structures of density perturbations associated with MCD SPW1, SPW2, and SPW3, respectively at $172 \mathrm{~km}$ altitude. This height is chosen due to its relevance to neutral mass spectrometer measurements from MAVEN. Figures 10(g), (h) and (i) show the same depictions for $108 \mathrm{~km}$. This altitude is chose since it is representative of altitudes where accelerometer-measured densities originated from MGS, MO and MRO. Amplitudes decrease with height for SPW1 ( $\sim 10-15 \%$ vs. $\sim 2-5 \%$ ), but increase with height for SPW2 and SPW3 ( $\sim 2-4 \%$ vs. $4-8 \%$ ). In addition, there are significant differences between the latitude-Ls structures between $108 \mathrm{~km}$ and $172 \mathrm{~km}$. Both of these features are consistent with the hypothesis that insitu generation by nonlinear wave-wave interactions is occurring in the thermosphere. It also implies that, at least insofar as SPW are concerned, longitude structures evident in aerobraking densities are not good indicators of what to expect in MAVEN mass spectrometer measurements.

3.5 Additional insights into thermosphere density variability Figure 11 presents latitude-Ls depictions of MCD DE3, DE2, DE1, SE1, S0 and D0 density perturbations at 108 and $172 \mathrm{~km}$. DE1 and DE2 are the dominant tides, each with 15-20\% density perturbations at 108 and $172 \mathrm{~km}$. At $108 \mathrm{~km}$, amplitudes associated with DE3, SE1, S0 and D0 are all of order 4-7\%, while at $172 \mathrm{~km}$ these tides generally contribute on the order of $5-12 \%$ each except for DE3 which is in the range $4-7 \%$.

Note that SW1 in our list of important solar-asynchronous tides is replaced here with D0, since D0 has about twice the amplitudes of SW1 at these altitudes. Examination of its height-latitude structures suggest that it does not originate to any significant degree in Mars' lower or middle atmosphere, but rather through nonlinear interactions with large-amplitude tides in the thermosphere:

$$
S P W 1 \times D W 1 \rightarrow D W 2+D 0
$$

$$
S P W 1 \times D E 1 \rightarrow D 0+D E 2
$$

$$
S P W 2 \times D E 2 \rightarrow D 0+D E 4
$$

As shown below, the aggregation of these various tidal components results in quite substantial $(\sim \pm 40 \%)$ total density perturbations in the thermosphere that are in line with, e.g., those derived from accelerometer measurements. 
In addition to the MCD-MCS comparisons in Figures 2, 5, and 8, the veracity of the MCD tidal structures is further checked by comparing longitude structures in the model against those revealed in MGS accelerometer measurements between 105-115 km in Figure 12. Consistent with the MCD model results in Figure 11, the MGS densities are cast in the form of percent residuals from the zonal mean within a window of time, and within each window the residuals are fit to sinusoids with respect to longitude with zonal wavenumbers $s=1,2,3,4$. That window is then moved through the data one sol at a time. It is desired that the window length be as short as possible so that time variations can to some degree be separated from latitude variations, yet still yield robust and relatively clean results for the individual wavenumber components. After trial and error, it was found that requiring the maximum spacing between any two adjacent longitudes in sequence within the window be less than $35^{\circ}$ in longitude was a good choice. It is also noted that in Figures 12-14, the percent density perturbations are now relative to the zonal-mean densities at the height, latitude and local time of the corresponding MGS densities.

Figure 12(a) illustrates the latitude-longitude structures obtained from the MGS densities according to the procedure described above, with Ls as a secondary y-axis. Locations of the individual periapsis data points are also included in Figure 12(a). Using the MCD-supplied software, the MCD model is interpolated and sampled at the height, latitude, longitude, LST and Ls of every MGS measurement location indicated in Figure 12(a), and analyzed identically to the MGS data to obtain Figure 12(b). Also shown in Figure 12(b) are the number of points in each fitting window, and the length of each fitting window, that result from the longitude sampling criterion described above. Figure 12(c) illustrates the latitude-longitude structure of density obtained from full latitude and longitude sampling of the MCD model at the heights, LSTs, and Ls values of the MGS measurements.

The main MGS feature (Figure 12(a)) that is well captured in both amplitude and phase by the sampled MCD (Figure 12(b)), is the wave-2 structure that extends from $50^{\circ} \mathrm{N}$ to $40^{\circ} \mathrm{S}$ in both figures. However, the maximum amplitudes near $60^{\circ}$ and $240^{\circ}$ longitude sometimes exceed $60 \%$ in both figures, whereas in Figure 12(c) the maximum amplitudes are in the same location except they are of order $40 \%$. This suggests that the excess amplitudes in Figures 12(a) and 12(b) are connected with the incomplete nature of MGS sampling. The tides that are capable of contributing to wave-2 in Figure 12(c), 
861

i.e., those with a space-based wavenumber $k_{s}=|s-n|=2$, include DE1 and S0. In the $\pm 30^{\circ}$ equatorial region, DE1 is the dominant contributor with S0 making a secondary contribution, as suggested by 108 km results in Figure 11. Based on Figure 7(c) which is applicable between $\mathrm{Ls}=60-90^{\circ}$, DE1 amplitudes are of order $12-20 \%$ between 110 $115 \mathrm{~km}$, the location of MGS periapsis during Ls $=45-85^{\circ}$. These values account for only about half the wave- 2 maxima/minima between $\pm 30^{\circ}$ in Figure 12(c), and suggests that the remainder might be accounted for in terms of the constructive/destructive interference effects of additional harmonics of the total longitudinal structure. Indeed, visual evidence of wave-3 exists in Figure 12(c) over much of the domain, and also wave-1 poleward of $40^{\circ} \mathrm{S}$. This interpretation is now pursued in connection with Figures 13 and 14 .

Figures 13 and 14 present the same three categories of plots as in Figure 12, except for longitudinal wave-1 and wave-2 in Figure 13, and wave-3 and wave-4 in Figure 14. Focusing on wave-2 for the moment, the MCD model indicates low-latitude maxima of order $15-22 \%$, consistent with the above interpretation that DE1 and S0 are the main contributors. Examination of DE1 and S0 in Figure 11 also indicate that these tidal components are the main contributors to the maxima poleward of $60^{\circ} \mathrm{S}$, consistent with the interpretation of polar-region MRO data by Forbes et al. (2018). Examination of MCD wave-3 and wave-4 amplitudes in Figures 14(c) and 14(f), respectively, indicate amplitudes of order $10-15 \%$, with maxima near $60^{\circ}$ and $240^{\circ}$ for wave- 4 and $90^{\circ}$ and $210^{\circ}$ for wave-3. These are fairly close to the wave-2 maxima in Figure 13(f), confirming that the aggregate effect of combining wave- 3 and wave- 4 to wave- 2 accounts for the difference in low-latitude amplitude maxima between Figure 12(c) and Figure 13(f).

Referring to Figures 10(a), 10(g), and 11(i), the primary contributor to wave-1 in Figures 13(b) and 13(c) is SPW1, with D0 making non-negligible contributions. This is also consistent with interpretations made by Forbes et al. (2018) in the context of polarregion MRO aerobraking density measurements. However, there is about a $150^{\circ}$ longitude shift compared to the maxima/minima in the S. Hemisphere of the MGS measurements in Figure 13(a). This accounts for some of the differences in the structures poleward of $40^{\circ} \mathrm{S}$ between Figures $12(\mathrm{~b})$ and $12(\mathrm{a})$. In addition, the MCD generally underestimates wave-1 amplitudes in the S. Hemisphere compared to MGS, which points to a deficiency of the model. 
The wave-3 MCD amplitudes in Figure 14(b) agree well in phase with those from MGS in Figure $14(\mathrm{a})$ between $30^{\circ} \mathrm{S}-55^{\circ} \mathrm{N}$, but underestimate amplitudes (roughly, 12 $15 \%$ vs. $15-25 \%)$. Based on Figures 7(b), 11(b), and 11(e), the main contributor to $k_{s}=$ $|s-n|=3$ at these latitudes is DE2 with non-negligible contributions from SE1. Poleward of $60^{\circ} \mathrm{S}$ SE1 likely assumes the primary role, and its signatures in Figure 14(b) also appear with similar amplitude and phase in the MGS density perturbations in Figure 14(a). The wave-3 density perturbations from MGS between $40^{\circ} \mathrm{S}-60^{\circ} \mathrm{S}$ are not replicated by MCD. The MCD wave- 4 densities in Figure $14(\mathrm{e})$ between $55^{\circ} \mathrm{S}-55^{\circ} \mathrm{N}$ similarly underestimate those from MGS in Figure 14(d), but the phases are again in quite good agreement except for the $25^{\circ}$ longitude phase difference near $50^{\circ} \mathrm{S}$. MCD DE3 amplitudes are of order $5-10 \%$ in this height regime at Ls $=60-90^{\circ}$ and between $20^{\circ} \mathrm{S}-50^{\circ} \mathrm{N}$, but SE2 amplitudes (not shown) are of order $3-5 \%$, extend between $40^{\circ} \mathrm{S}-60^{\circ} \mathrm{N}$, and are thus not negligible in comparison. We conclude that most of the structures seen in Figures 14(d) and $14(\mathrm{e})$ between $\pm 55^{\circ}$ latitude are attributable to the combinations of DE3 and SE2.

\section{Summary and Conclusions}

In the first part of this paper, Mars Climate Sounder (MCS) temperature measurements at $76 \mathrm{~km}$ were used to construct a multi-year latitude versus Ls climatology of the tidal spectrum. The most important spectral components included the solar-synchronous ("migrating") components DW1, SW2, and the solar-asynchronous ("non-migrating") tides DE3, DE2, DE1, SE1, S0, and SW1. A similar analysis was performed on model output from the Mars Climate Database, and the same tidal components arose as the most significant. The largest tidal components were found to be DW1 and SW2. The MCS amplitudes of DW1 and SW2 were comparable ( 4-9K), whereas MCD amplitudes were somewhat larger $(\sim 6-14 \mathrm{~K})$ for DW1 and smaller for SW2 $(\sim 3-7 \mathrm{~K})$. Assessment of the veracity of MCD with regard to SW2 at latitudes equatorward of $\pm 40^{\circ}$ latitude is impeded by the particularly sparse local time sampling of MCS at these latitudes, which introduces an unknown level of uncertainty due to potential aliasing with the zonal- and diurnal-mean component of temperature. Discrepancies between the measured and modeled estimates of DW1 potentially arise from an underestimation of water-ice cloud radiative forcing in the MCD, and possibly the zonal-mean zonal winds $(\bar{U})$ in MCD. The height-latitude structures (0-170 km, pole-to-pole) of DW1 and SW2 were examined within the context of the MCD. Emphasis was placed on a few typical examples 
that revealed the influences of $\bar{U}$ and dissipation on these structures. DW1 and SW2 responded to eastward $\bar{U}^{\prime}$ s at middle to high latitudes through Doppler-shifting to higher frequencies, latitudinal broadening, lengthening of their vertical wavelengths, and reduced dissipation. The vertical wavelength of DW1 at low-middle latitudes is of order $50 \mathrm{~km}$, as compared with $32 \mathrm{~km}$ from classical tidal theory. As shown by Takahashi et al. (2006), this can be traced to the meridional shear in $\bar{U}$, as embodied in the zonal-mean vorticity $\bar{\zeta}$ term in the zonal momentum equation (see also McLandress (2002) for other effects on DW1 due to $\bar{\zeta}$ at Earth). Furthermore, the zonal-mean westward jet, which is a prominent feature at low latitudes above $80-100 \mathrm{~km}$ in Mars atmosphere, represented an impediment to vertical propagation, so that the solar-synchronous tides tend to propagate within propagation channels bounded by this westward jet and the poles. Dopplershifting and $\bar{\zeta}$ effects on tides at Mars are much more extreme than in Earth's atmosphere, due to Mars' smaller radius and the more intense $\bar{U}^{\prime}$ s that exist in its atmosphere.

Significantly better MCD-MCS agreement in terms of seasonal-latitudinal structures and amplitudes were found for the solar-asynchronous tidal components. This led us to examine height-latitude structures of their density perturbations with respect to the zonal mean, in light of their relevance to interpretation of accelerometer-measured densities from MGS, MO and MRO, as well as mass spectrometer measurements on MAVEN. The largest solar-asynchronous tides, DE3, DE2 and DE1, are all eastward-propagating and confined to low latitudes. The $\bar{U}$ distribution therefore favored vertical propagation, so that quite large density perturbations $(\sim 15-25 \%)$ were attainable in the $100-170 \mathrm{~km}$ height region. These large amplitudes also led to the secondary generation of tides through tide-tide and tide-SPW1 nonlinear interactions (for instance, D0 at 5-10\%), and to the in-situ production of SPW in the thermosphere. Amplitudes of order $5-10 \%$ were found for SPW2 and SPW3 at $170 \mathrm{~km}$. SE1 and S0 amplitudes were found to range between 10 and $20 \%$ at $170 \mathrm{~km}$ altitude, whereas SW1 was much smaller in general $(<5 \%)$.

The net effect of these solar-asynchronous tides and SPWs is that in aggregation their constructive/destructive interference can lead to significant longitudinal variability in addition to local time variability. To further test the veracity of the MCD in this regard, a comparison between MCD density perturbations and those derived from PhaseII aerobraking of MGS was performed. The MGS latitude $\left(55^{\circ} \mathrm{N}\right.$ to $\left.40^{\circ} \mathrm{S}\right)$ versus longitude structure sampled between $\mathrm{Ls}=40^{\circ}$ and $\mathrm{Ls}=80^{\circ}$ is dominated by a wave- 2 structure with amplitudes as large as $\pm 65 \%$. The MCD comparison was performed with MCD 
sampled according to the same heights, latitudes, LSTs, longitudes and Ls values as MGS, and analyzed identically. In addition, a comparison was made with the same latitudelongitude structure obtained from full sampling of the MCD. This allowed isolation of the influences of sampling from those of the actual underlying tides.

First of all, the MGS-sampled MCD captured the dominant wave-2 structure in MGS very well in phase, and achieved almost as large maximum amplitudes. However, these were significantly larger than the $\sim \pm 45 \%$ amplitudes from the fully-sampled MCD, indicating that some of the former originated from high-order modes and/or day-to-day variability not captured by the incomplete nature of the MGS sampling. Furthermore, the $\pm 45 \%$ amplitudes from the fully-sampled MCD were much larger than those contributed by DE1 itself $(\sim \pm 20 \%)$, which at first sight and in many prior publications is the assumed interpretation of the origin of wave-2. The deficit was in fact found in wave-3 and wave-4 (due mainly to DE2 and DE3, respectively), which combined synergistically with DE1 to nearly replicate the larger-amplitude "wave-2" structure in the MGS-sampled MCD and in the MGS observations themselves.

Poleward of $40^{\circ} \mathrm{S}$ the MGS data and the MCD both revealed wave-2 density perturbations of order $\sim 10 \%$, similar in phase. It was determined that this feature originated from a combination of DE1 and S0. MGS and MCD also indicated 15-20\% wave1 density perturbations in the same latitude regime, but with about a $150^{\circ}$ longitudinal shift in phase. The primary contributor to wave-1 was concluded to be SPW1, with D0 making non-negligible contributions. These same conclusions regarding origins of wave1 and wave-2 were made by Forbes et al. (2018) in the context of interpreting MRO aerobraking data at high S. Hemisphere latitudes around Ls $\approx 80^{\circ}$.

With regard to the solar-asynchronous tides that give rise to significant longitudinal variability in the thermosphere of Mars, it is concluded that MCD captures the salient amplitude and phase characteristics of the $\sim \pm 30-60 \%$ longitudinal density perturbations measured by the MGS accelerometer near $105-115 \mathrm{~km}$, and the seasonal-latitudinal variability of the tidal spectrum derived from MCS temperature measurements at $76 \mathrm{~km}$. This makes credible the interpretations contained herein regarding the height-latitude structures of various tidal components between 76 and $170 \mathrm{~km}$. Additional assessments of the ability of MCD to replicate longitude structures from MO, MRO, and MAVEN are planned for future work. 
989

Finally, despite the impressive agreement between the MCD and MRO/MCS and MGS accelerometer data demonstrated herein, noteworthy differences and knowledge gaps remain. The $\bar{U}$ distribution in the middle and upper atmosphere of Mars is not well constrained by existing observations, and moreover is likely to be affected by gravity waves (e.g., Medvedev et al., 2011) whose properties in Mars' atmosphere are also not well constrained. Some recent progress on incorporating non-orographic gravity wave effects into the LMD GCM used to produce the MCD have recently been made (Gilli et al., 2020), and will be incorporated into future MCD versions. As acknowledged above, tidal forcing by radiatively active water-ice clouds warrants closer examination in the LMD GCM, and because this source is modulated by topography, future modifications will likely influence both solar-synchronous and solar-asynchronous tides. Similarly, the GCM is also known to underestimate the vertical extension of the dust and in particular the formation of detached layers in the atmosphere (see e.g. Wang et al., 2018), and efforts are ongoing to better represent the dust cycle (alongside the water cycle) in the GCM. Asthe absorption of heat by dust is one of the primary sources of thermal tides, this should also affect all modeled tides and in particular the partitioning between diurnal and semidiurnal tides which is controlled by the depth of the heat absorption.

Recent successes at Earth in terms of high-resolution whole-atmosphere models that enable resolution of gravity waves and their effects (e.g., Liu et al., 2014), and that assimilate observational data (e.g., Pedatella et al., 2014), may point the way for future Mars model developments. Indeed the present paper suggests that assimilation of MCSlike middle-atmosphere measurements into a whole-atmosphere Mars model could represent a leap forward in our ability to predict thermosphere longitude structures due to solar tides. While significant advances have been made insofar as data assimilation of low- and middle-atmosphere data goes (Navarro et al., 2017; Graybush et al., 2019), it remains to be determined how thermosphere structures are affected. Concerning the need for future observations, major advances would likely result from improved local time sampling of Mars atmosphere. This could be accomplished by more than one satellite precessing in local time, or 2-4 satellites in near-polar orbits sampling 4-8 local times simultaneously (see, e.g., Kleinböhl, et al., 2018). Small satellites and new technologies now make these scenarios feasible. 
(b) Latitude $=0^{\circ}$, Longitude $=240^{\circ}, \mathrm{Ls}=120^{\circ}$

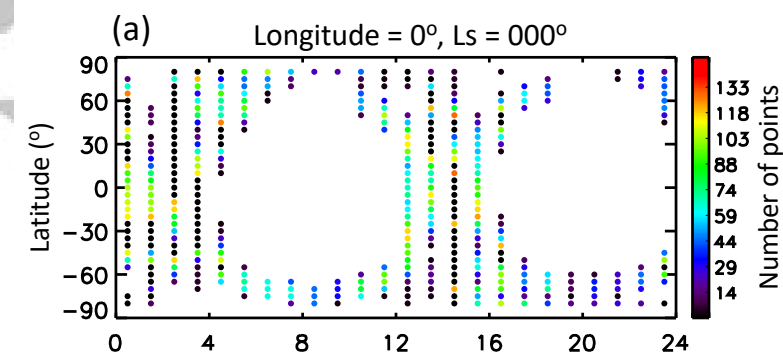

(c) Latitude $=40^{\circ}$, Longitude $=240^{\circ}, \mathrm{LS}=000^{\circ}$

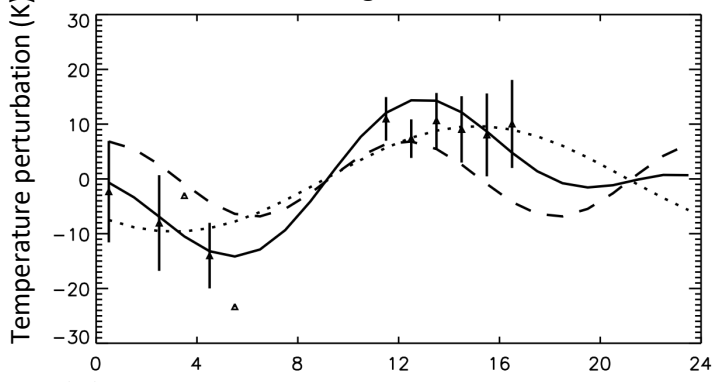

(e) Latitude $=-40^{\circ}$, Longitude $=240^{\circ}, \mathrm{Ls}=000^{\circ}$

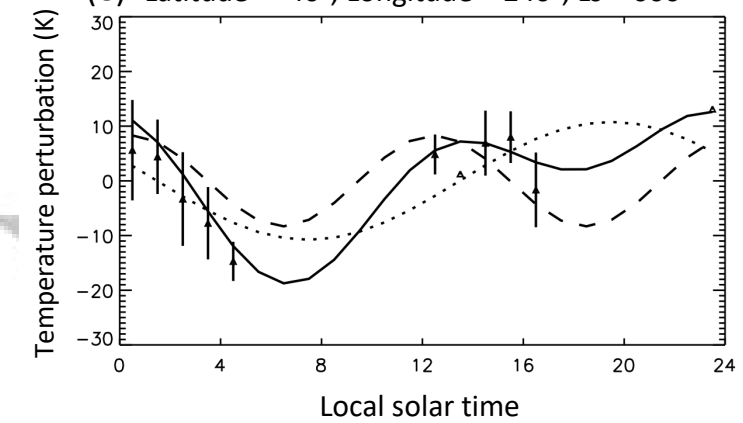

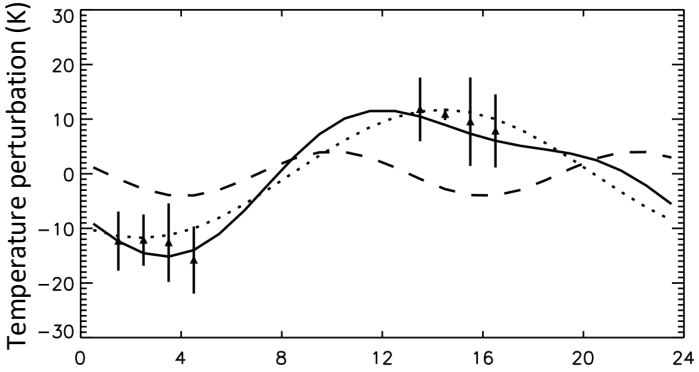

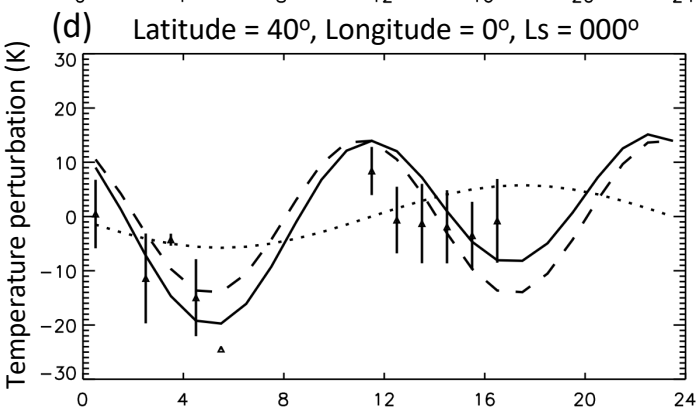

(f) Latitude $=80^{\circ}$, Longitude $=240^{\circ}$, Ls $=000^{\circ}$

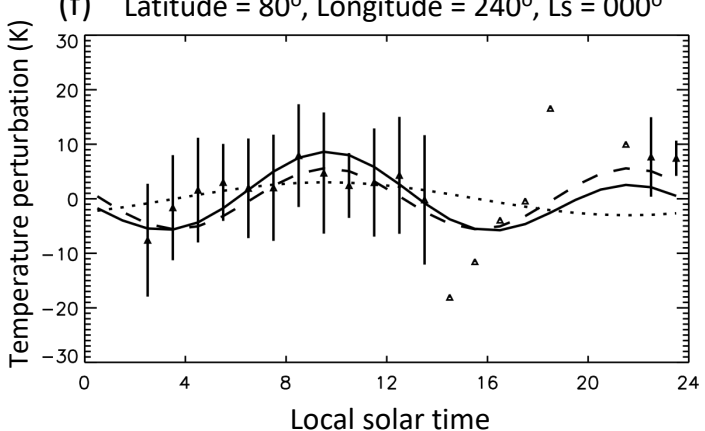

Figure 1. Typical examples of (a) latitude-local time data distributions, and (b)-(f) tidal

fits. In (b)-(f) dotted lines are diurnal fits, dashed lines are semidiurnal fits, and solid lines are combined diurnal and semidiurnal fits. The data points in the fits are weighted according to the cube root of the number of points in the corresponding local time bin at a given latitude and longitude. The data points without error bars are single data points. 

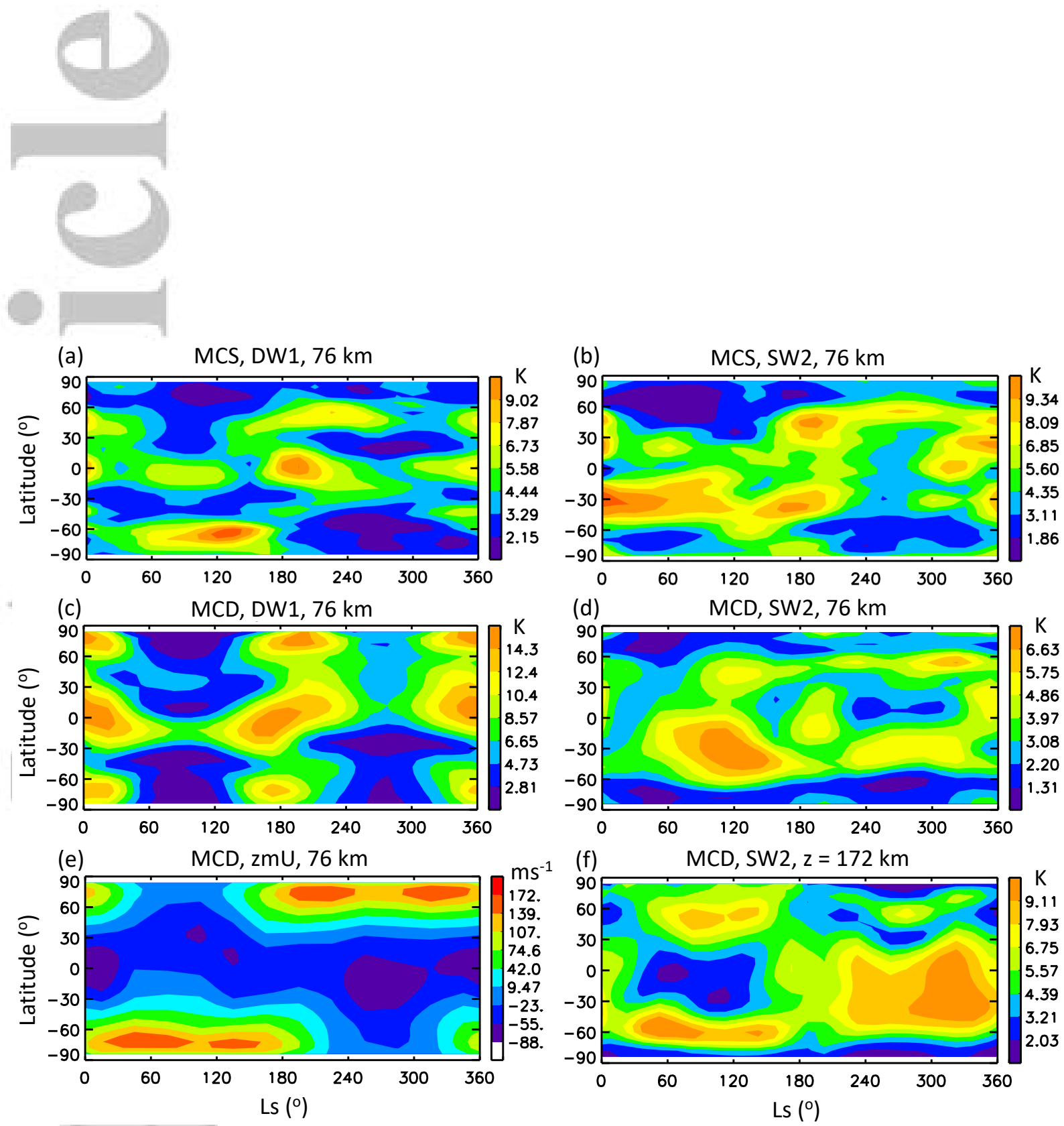

Figure 2. (a) and (b): Latitude-Ls variability of MCS DW1 and SW2 temperature amplitudes at $76 \mathrm{~km}$ above Mars' areoid. (c) and (d): Same as (a) and (b) except for MCD. (e) Latitude-

Ls variation of zonal-mean zonal winds from MCD at $76 \mathrm{~km}$. (f) Latitude-Ls variation of SW2 


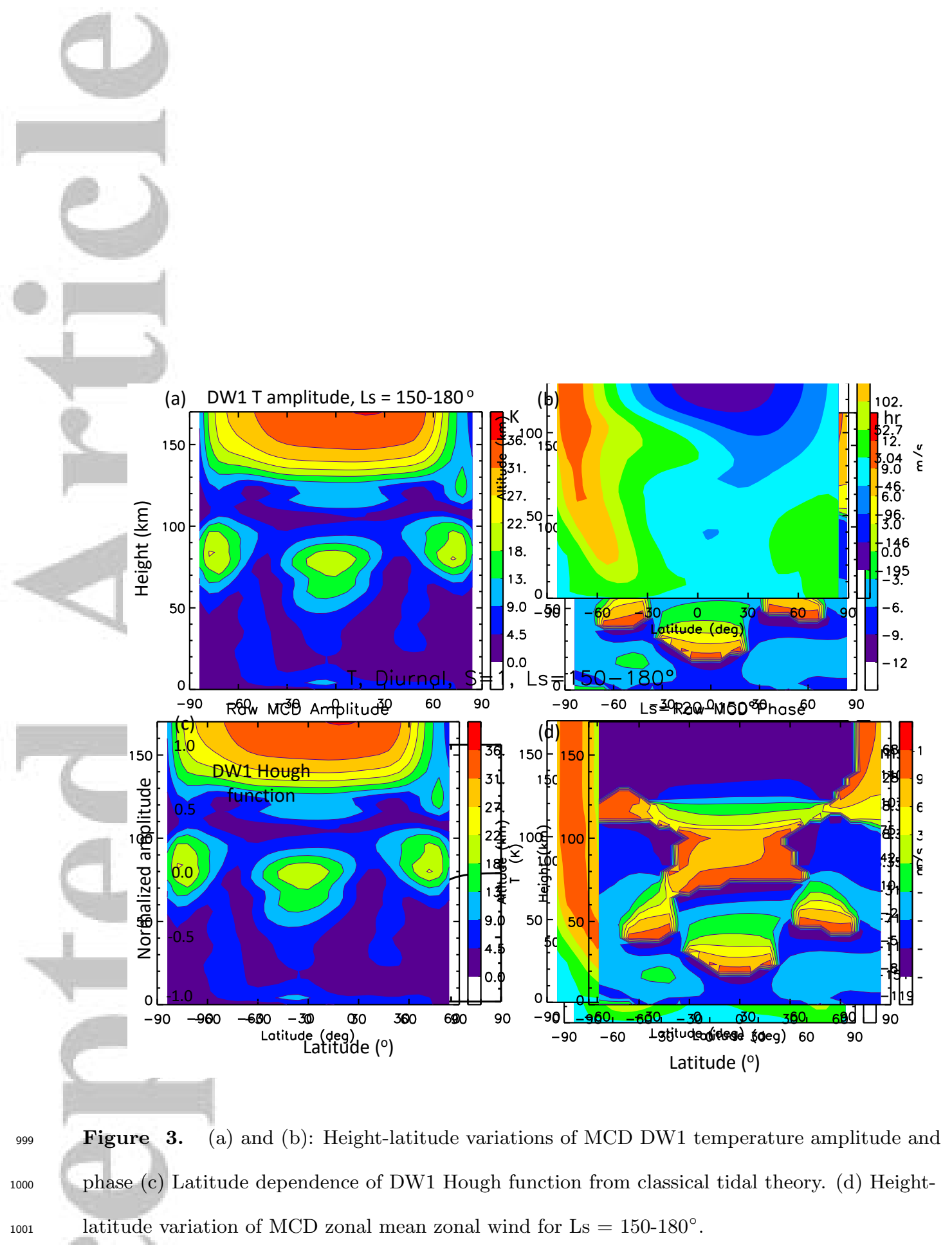



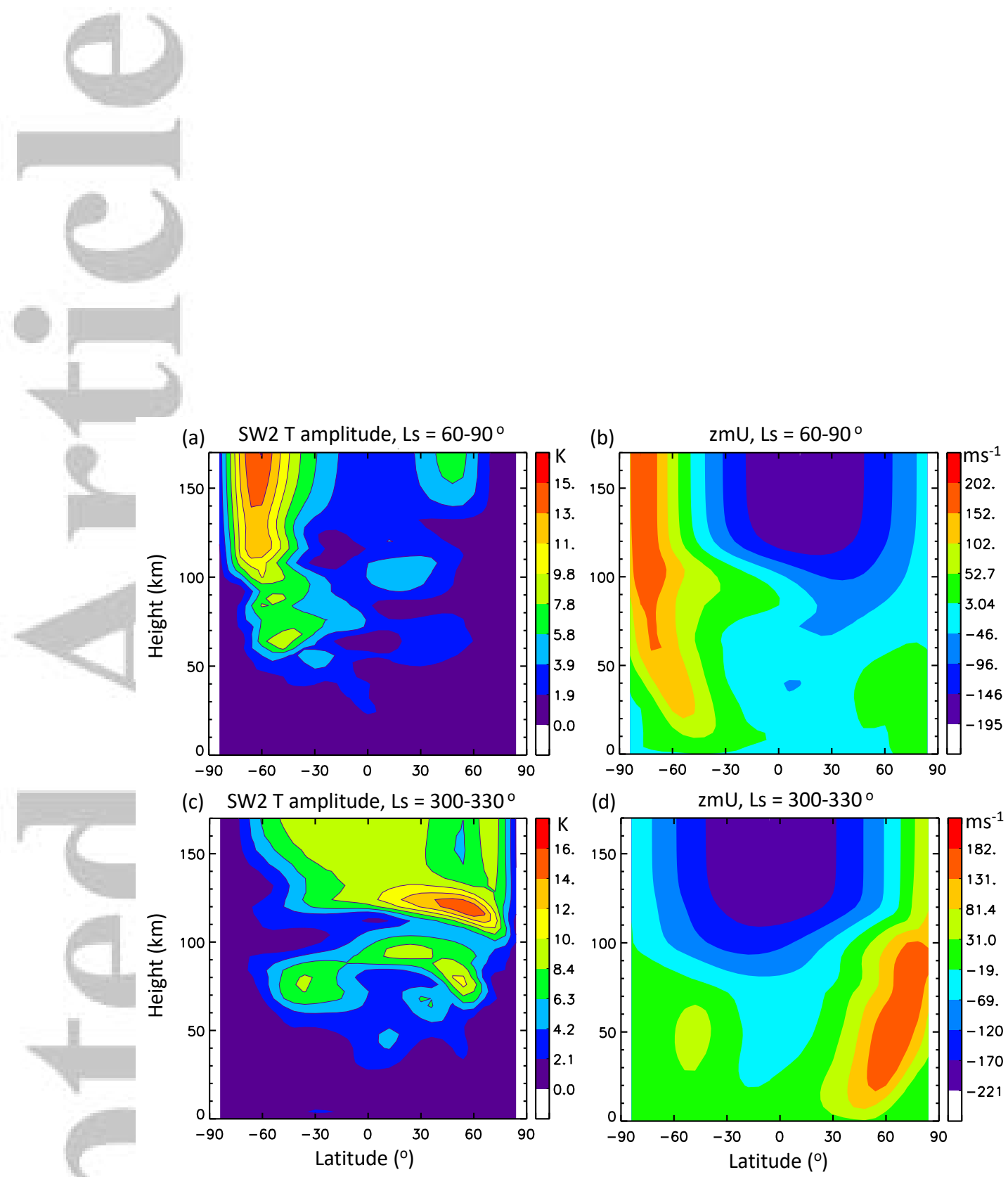

(d) $\quad \mathrm{zmU}, \mathrm{Ls}=300-330^{\circ}$



Figure 4. (a) and (b): Height-latitude variation of MCD SW2 temperature amplitude and

MCD zonal-mean zonal wind, for $\operatorname{Ls}=60-90^{\circ}$. (c) and (d): Same as (a) and (b) except for $\mathrm{Ls}=$ 1004 $300-330^{\circ}$. 

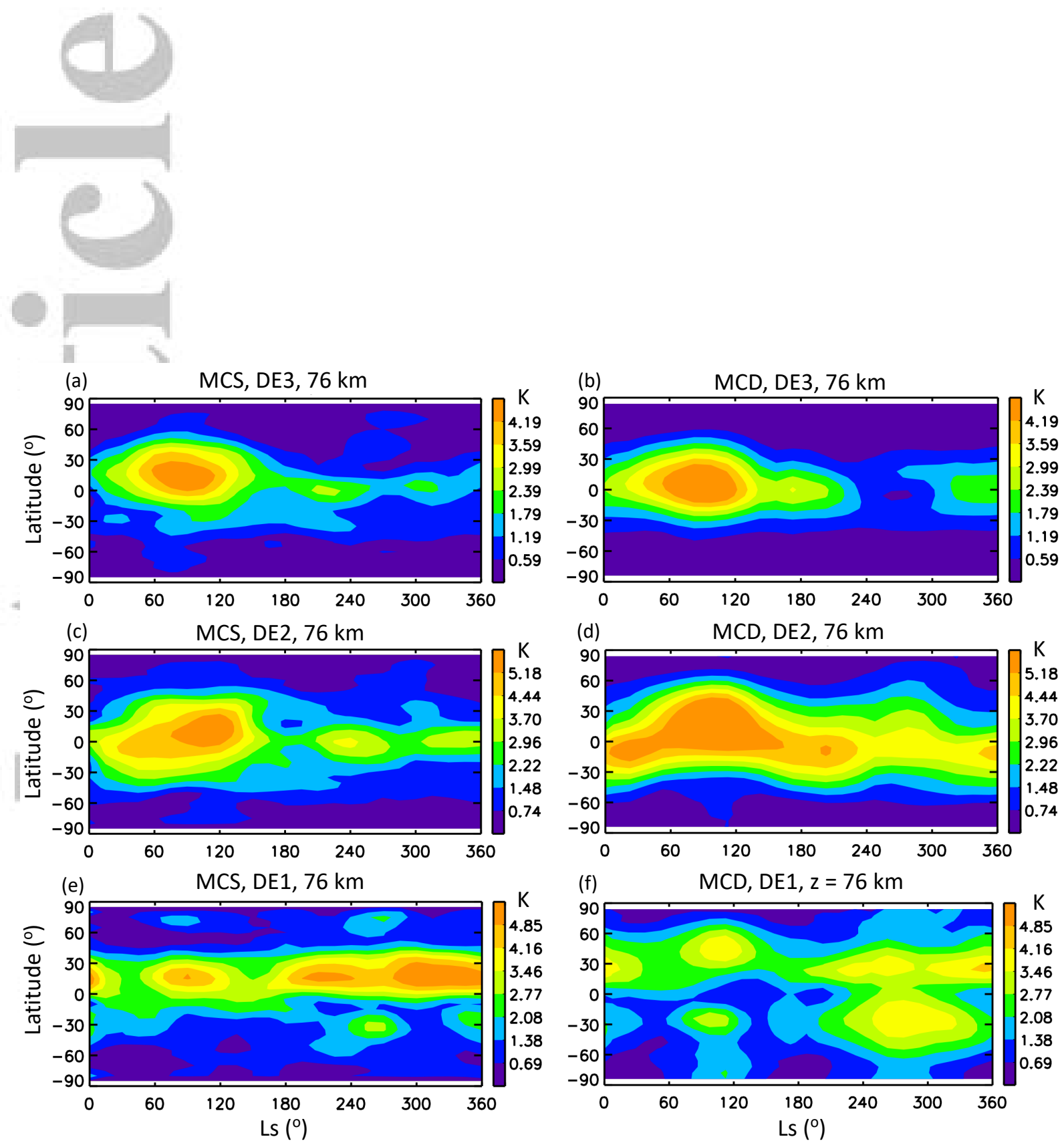

Figure 5. (a) and (b): Latitude-Ls variations of MCS and MCD DE3 temperature ampli-

tudes at $76 \mathrm{~km}$ above Mars' areoid. (c) and (d): Same as (a) and (b) except for DE2. (e) and (f):

Same as (a) and (b) except for DE1. 

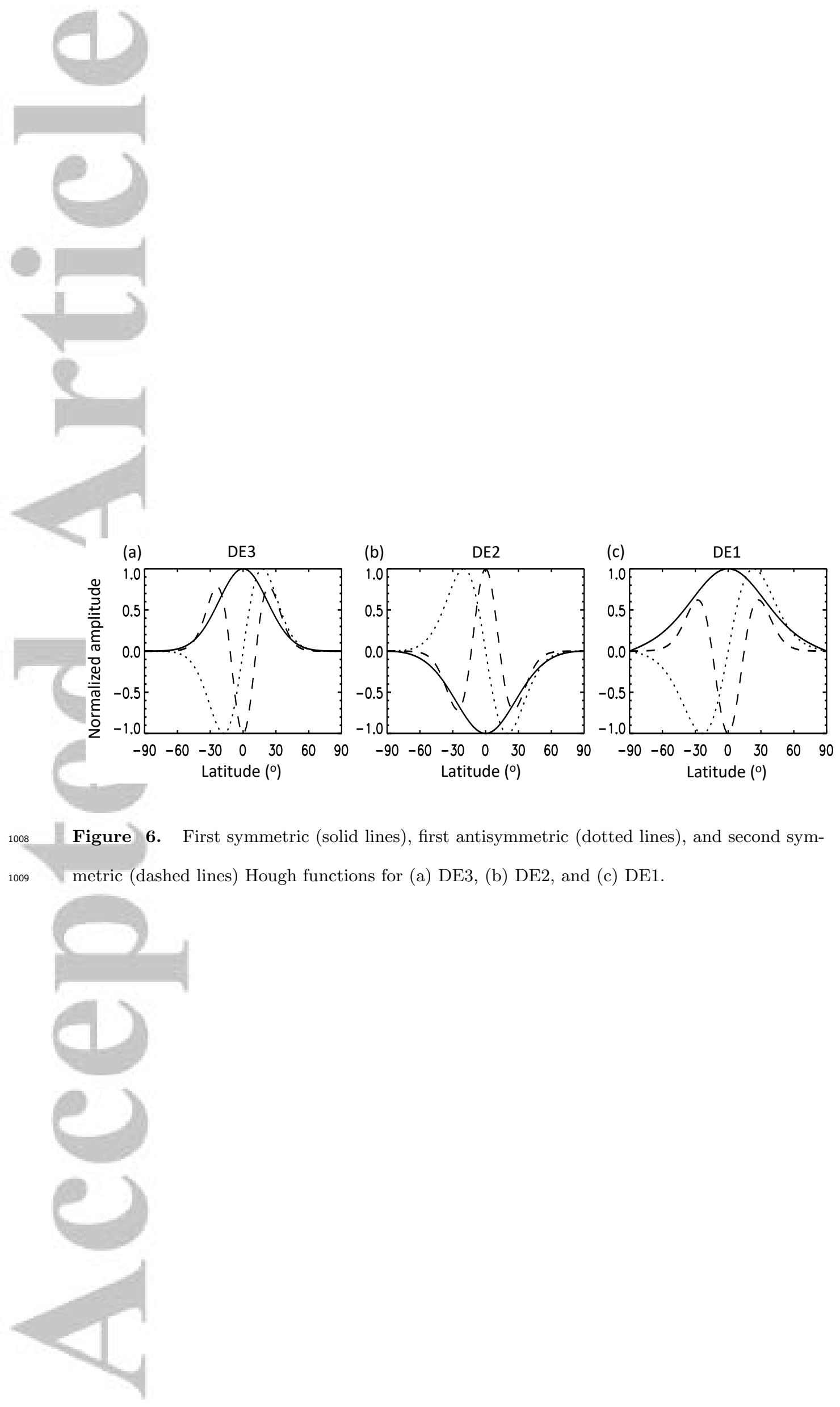

1008 Figure 6. First symmetric (solid lines), first antisymmetric (dotted lines), and second sym-

1009 metric (dashed lines) Hough functions for (a) DE3, (b) DE2, and (c) DE1. 
(a) DE3 $\Delta \rho / \rho_{0}$ amplitude, Ls $=60-90^{\circ}$

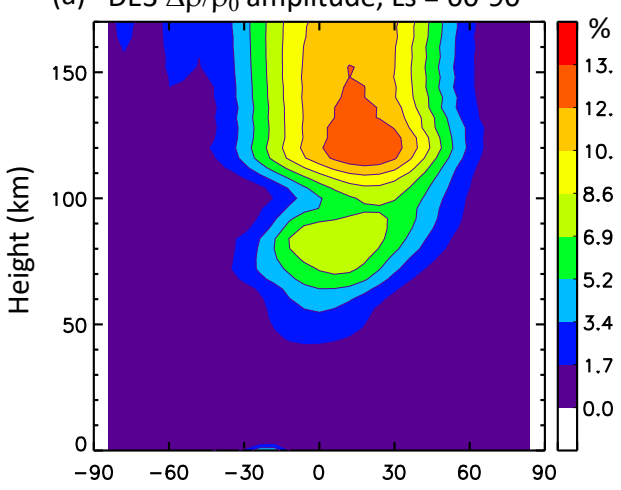

(c) $\mathrm{DE} 1 \Delta \rho / \rho_{0}$ amplitude, $\mathrm{Ls}=60-90^{\circ}$

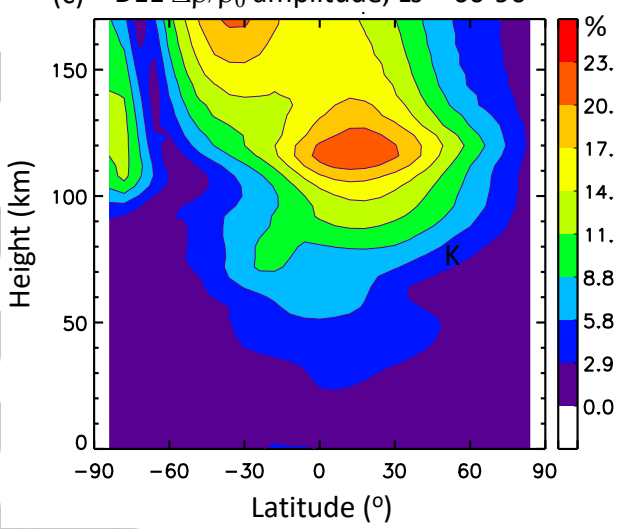

(b) DE2 $\Delta \rho / \rho_{0}$ amplitude, Ls $=60-90^{\circ}$

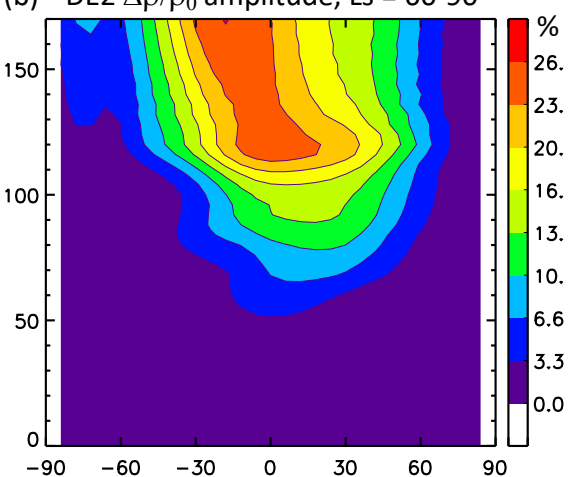

(d)

$\mathrm{zmU}, \mathrm{Ls}=60-90^{\circ}$

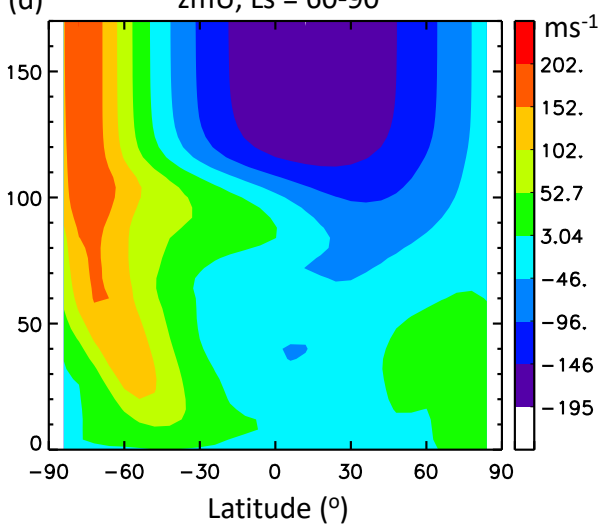

Figure 7. Height-latitude structures of MCD \% relative density perturbations for Ls $=60-90^{\circ}$ for (a) DE3, (b) DE2, and (c) DE1. Zonal-mean zonal winds are shown in panel (d).

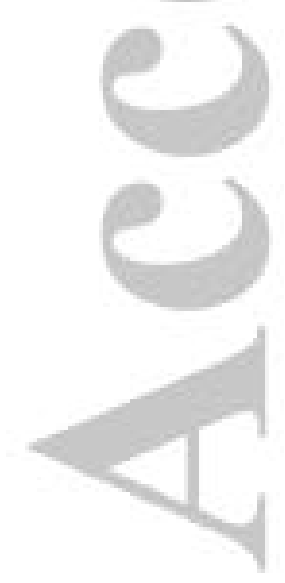



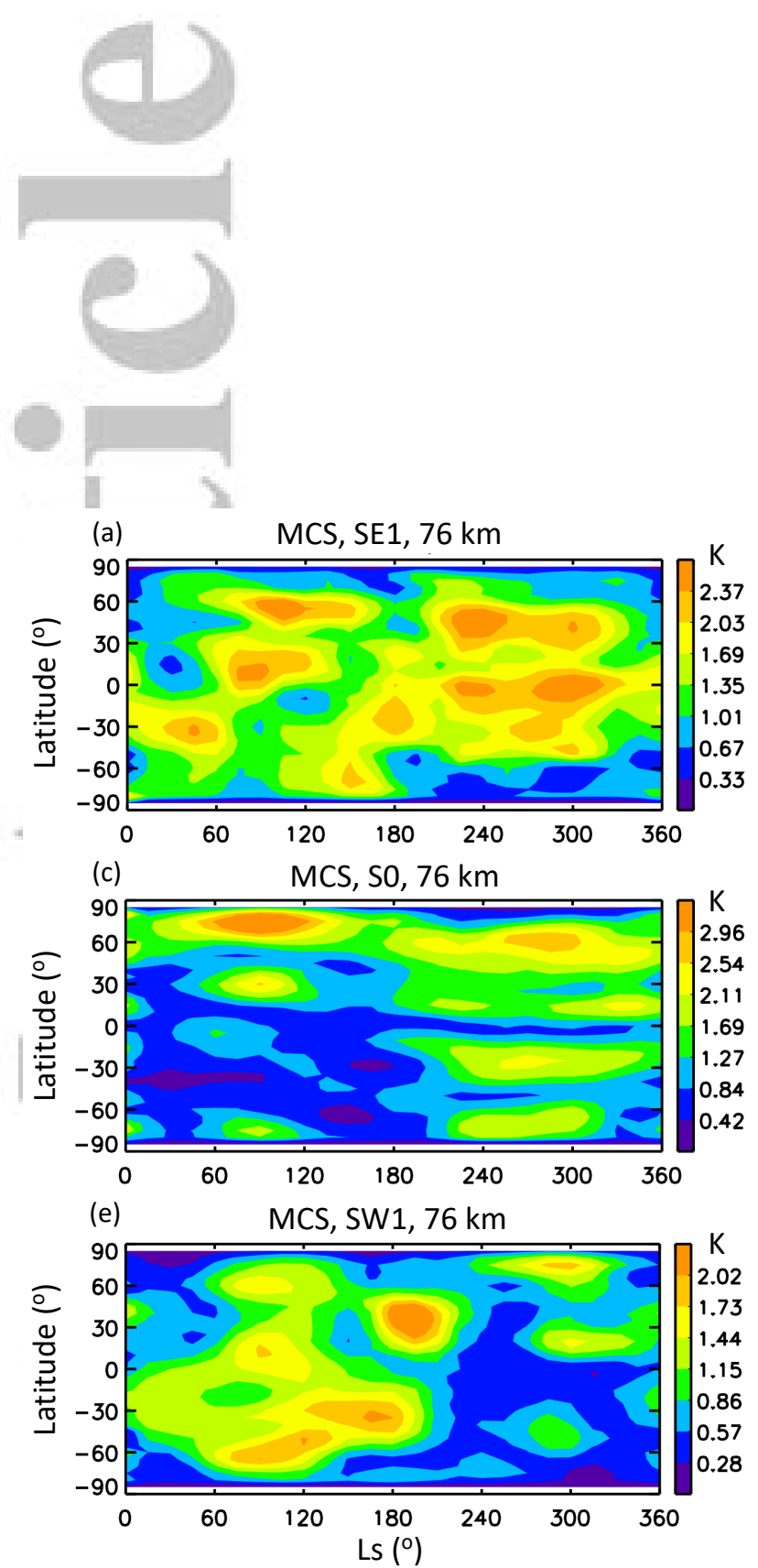
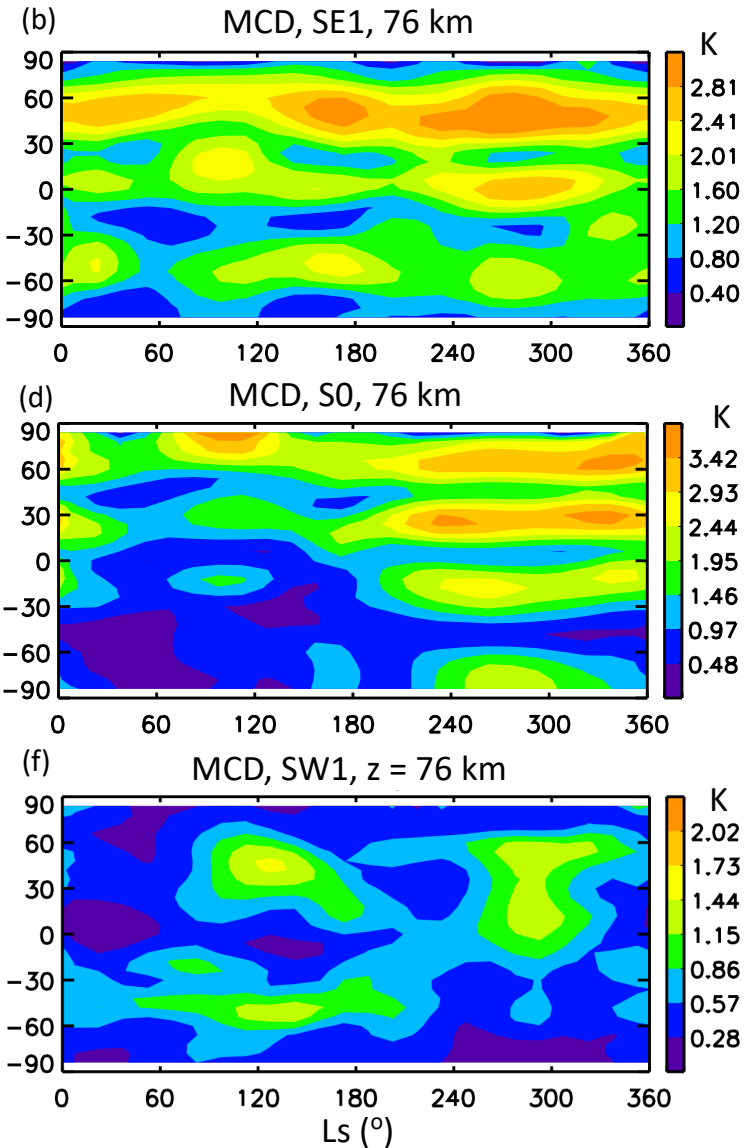

Figure 8. (a) and (b): Latitude-Ls variations of MCS and MCD SE1 temperature amplitudes at $76 \mathrm{~km}$ above Mars' areoid. (c) and (d): Same as (a) and (b) except for S0. (e) and (f): Same as (a) and (b) except for SW1. 


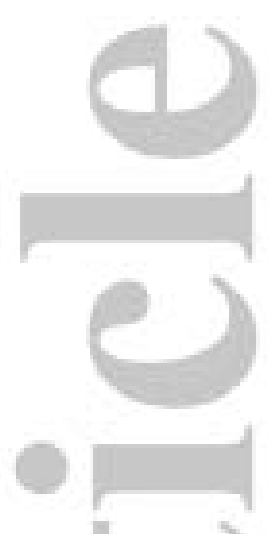

(a) SE1 $\Delta \rho / \rho_{0}$ amplitude, $\mathrm{Ls}=240-270^{\circ}$
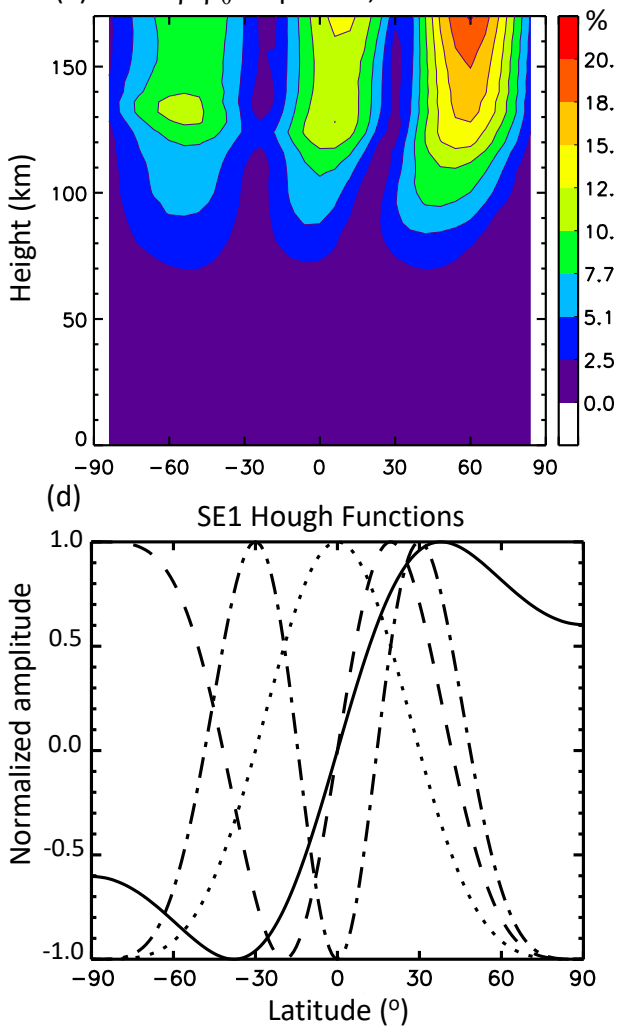

(b) So $\Delta \rho / \rho_{0}$ amplitude, $L s=240-270^{\circ}$

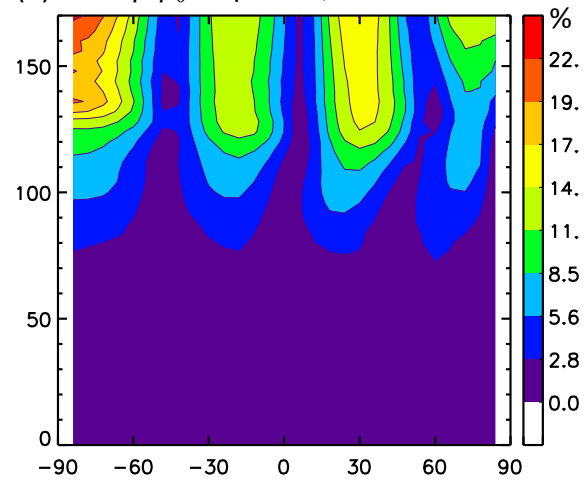

(e)

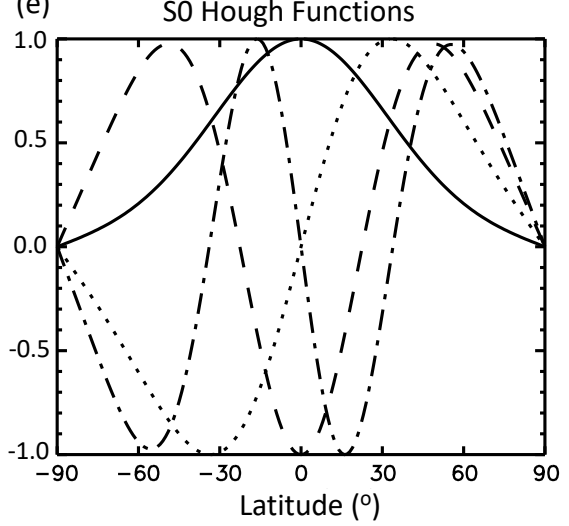

(c) SW1 $\Delta \rho / \rho_{0}$ amplitude, Ls $=240-270^{\circ}$
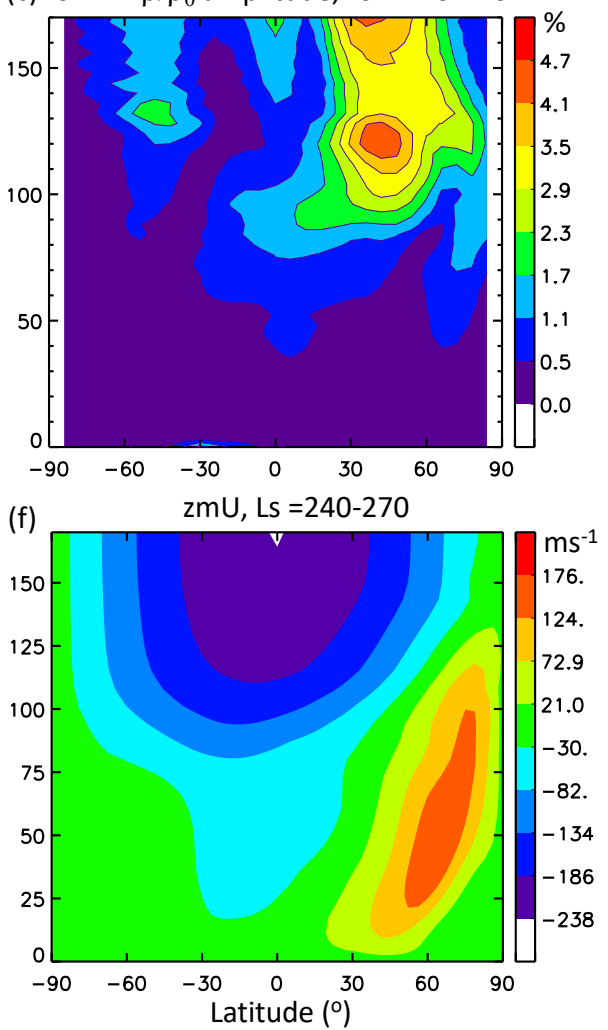

Figure 9. Height-latitude structures of MCD \% relative density perturbations for Ls $=240$ -

$270^{\circ}$ for (a) SE1, (b) S0, and (c) SW1. Zonal-mean zonal winds are shown in panel (f). First four

SE1 and S0 Hough functions are shown in panels (d) and (e): first symmetric (solid lines), first antisymmetric (dotted lines), second symmetric (dashed lines), second antisymmetric (dasheddotted lines). 



(c) SPW3, Ls 60-90。
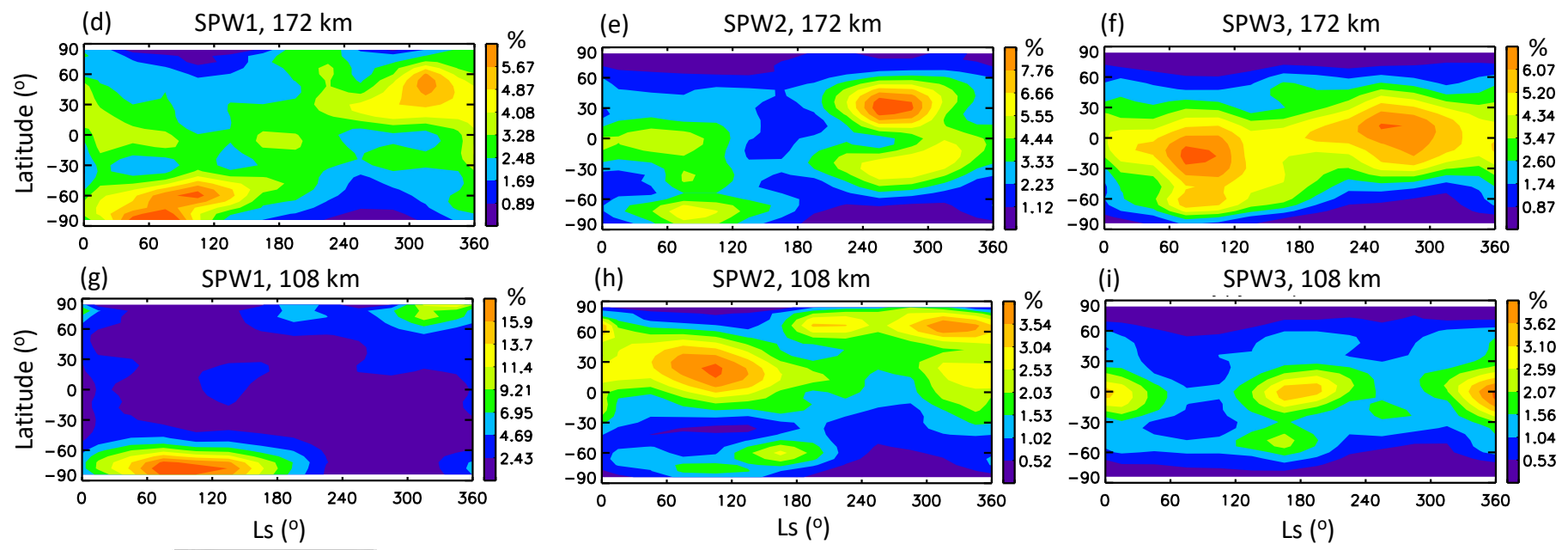

Figure 10. Sample depictions of MCD \% relative density perturbations for SPW1 (left),

${ }_{1021}$ SPW2 (middle), and SPW3 (right). (a), (b) and (c): height-latitude structures for Ls $=60-90^{\circ}$.

1022 (d), (e) and (f): latitude-Ls structures at $172 \mathrm{~km} .(\mathrm{g}),(\mathrm{h})$ and (i): latitude-Ls structures at 108

$1023 \mathrm{~km}$. 

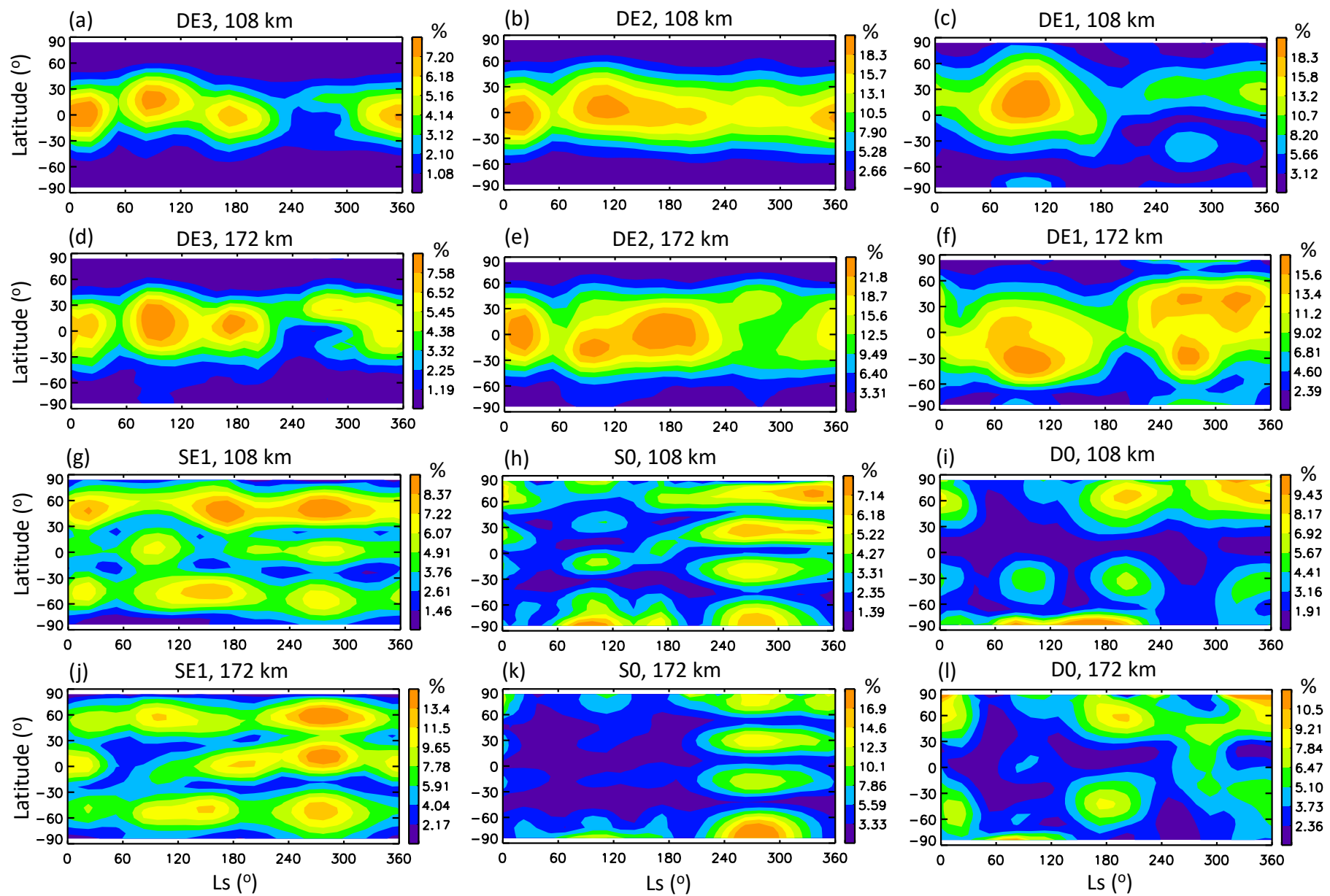

1024 Figure 11. Latitude-Ls distributions of MCD \% relative density perturbations. (a), (b) and

1025 (c): DE3, DE2, and DE1 at $108 \mathrm{~km} .(\mathrm{d}),(\mathrm{e})$ and (f): DE3, DE2, and DE1 at $172 \mathrm{~km} .(\mathrm{g}),(\mathrm{h})$

1026 and (i): SE1, S0 and D0 at $108 \mathrm{~km}$. (j), (k) and (l): SE1, S0 and D0 at $172 \mathrm{~km}$. 


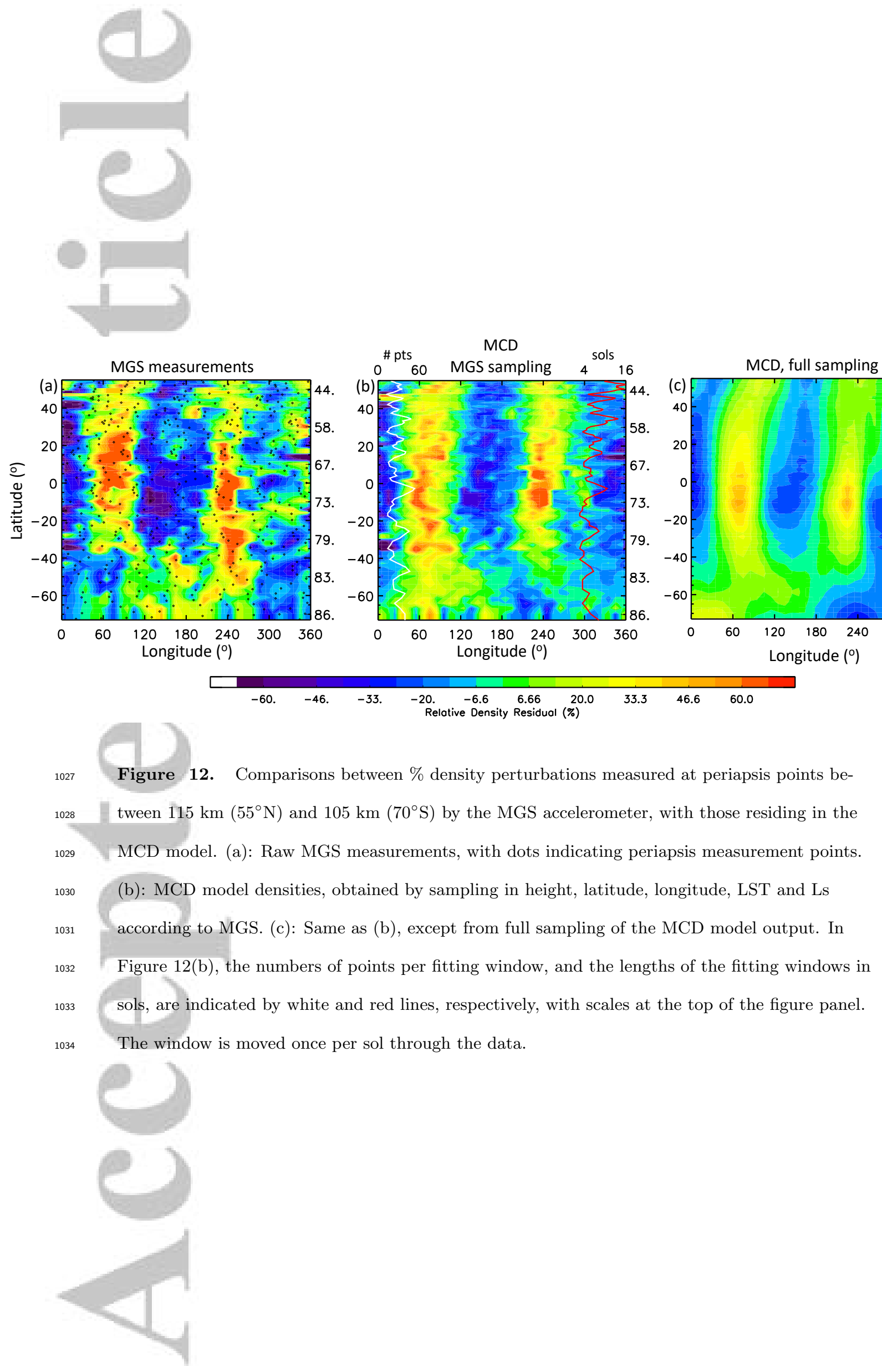




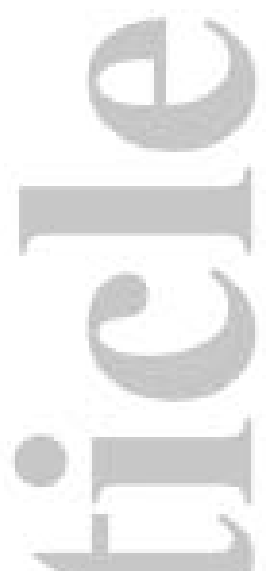

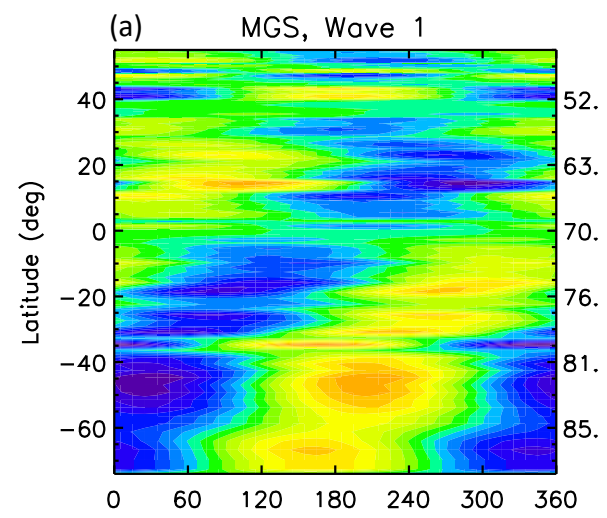

(d)

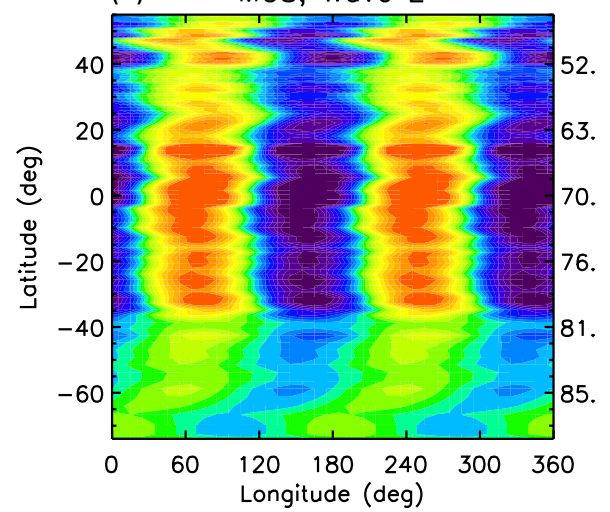

(b) sampled MCD, Wave 1

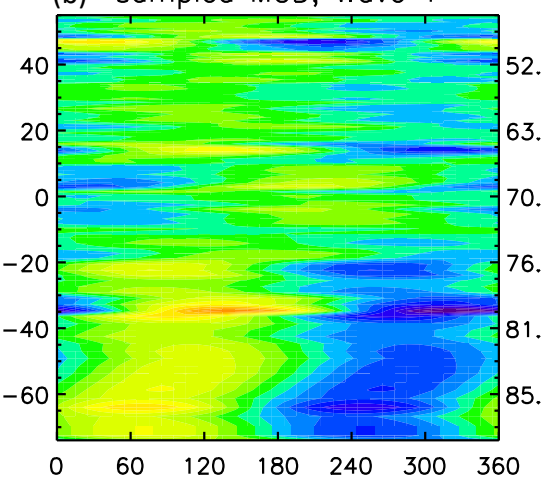

(e) sampled MCD, Wave 2



(c) full MCD, Wave 1

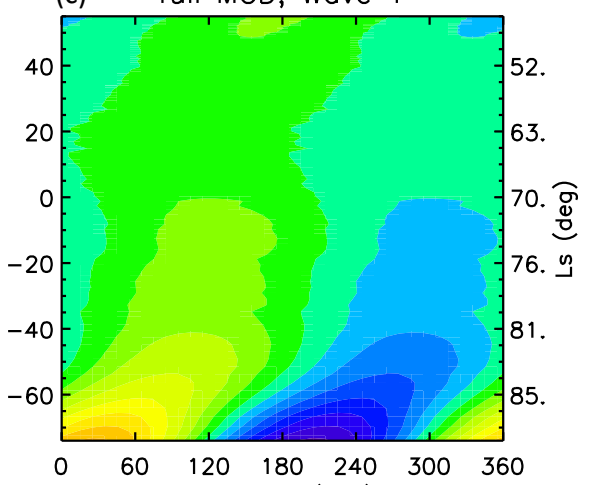

(f) full MCD, Wave 2

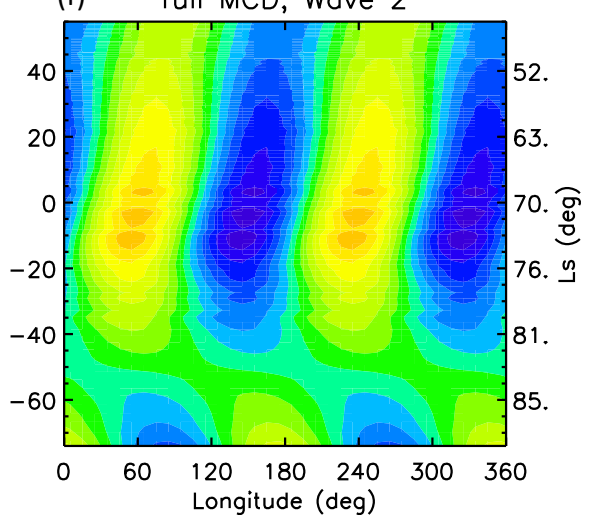

$-30 .-23$

$\begin{array}{llllllll}\text { 16. } & -10 . & -3.3 & 3.33 & 10.0 & 16.6 & 23.3 & 30.0\end{array}$

Phase II Relotive Density Residual (\%)

1035 Figure 13. Same as Figure 12, except for longitudinal wave-1 (top row) and wave-2 (bottom

1036 row). 


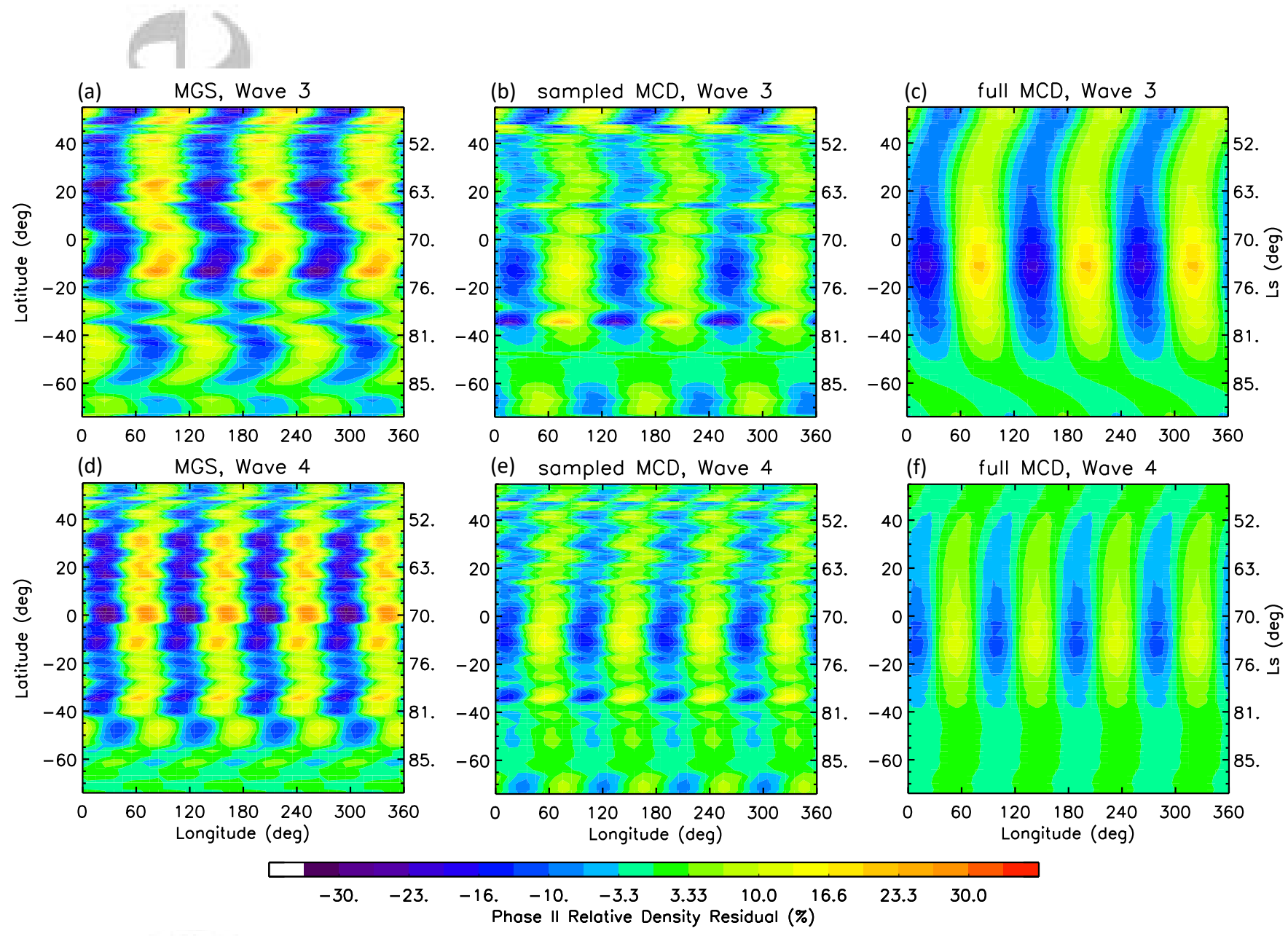

Figure 14. Same as Figure 12, except for longitudinal wave-3 (top row) and wave-4 (bottom row).

\section{Acknowledgments}

This work was supported by NASA Grant NNX16AJ39G through the Mars Data Analysis Program (MDAP) to the University of Colorado. The LMD team acknowledges support from CNES and ESA. Work at the Jet Propulsion Laboratory, California Institute of Technology, is performed under contract with the National Aeronautics and Space Administration. The MCS and MGS data were obtained from the Planetary Data System at http://pds-atmospheres.nmsu.edu/PDS/data. The Mars Climate Database (MCD) is publicly available at http://www-mars.lmd.jussieu.fr. The authors thank David Kass for providing Figure S1 in the supplemental material of this publication, and also thank self-identified reviewers R. John Wilson and Richard Zurek for their valuable comments on the initial submission of this manuscript. 


\section{References}

Andrews, D.G., J.R. Holton \& C.B. Leovy (1987). Middle Atmosphere Dynamics. Academic Press, 489 pp.

Angelats i Coll, M., \& J.M. Forbes (2002). Nonlinear interactions in the upper atmosphere: The $s=1$ and $s=3$ nonmigrating semidiurnal tides. J. Geophys. Res., 107, 1157, doi:10.1029/2001JA900179

Angelats i Coll, M., Forget, F., Lòpez-Valverde, M.A., Read, P.L., \& S.R. Lewis (2004). Upper atmosphere of Mars up to $120 \mathrm{~km}$ : Mars Global Surveyor accelerometer data analysis with the LMD general circulation model. J. Geophys. Res., 109, doi:10.1029/2003JE002163. Bougher, S.W., Ridley, A., Pawlowski, D., Bell, J.M. \& S. Nelli (2011). Development and validation of the ground-to-exosphere Mars GITM code: Solar cycle and seasonal variations of the upper atmosphere, in Mars Atmosphere: Modelling and Observation, edited by F. Forget and E. Millour, 379-381.

Bougher, S.W., Pawlowski, D. Bell, J.M., Nelli, S., McDunn, T., Murphy, J.R., Chizek, M. \& A. Ridley (2015). Mars Global Ionosphere-Thermosphere Model: Solar cycle, seasonal, and diurnal variations of the Mars upper atmosphere. $J$. Geophys. Res. Planets, 120, 311-342, doi:10.1002/2014JE004715 Chapman, S., \& R.S. Lindzen (1970). Atmospheric Tides: Thermal and Gravitational, Gordon and Breach, New York.

Conrath, B.J. (1976). Influence of planetary-scale topography on the diurnal thermal tide during the 1971 Martian dust storm. J. Atmosphere. Sci., 33, 2430-2439. doi:10.1175/1520-0469(1976)033<2430:IOPSTO >2.0.CO;2 England, S.L., et al. (2016). Simultaneous observations of atmospheric tides from combined in situ and remote observations at Mars from the MAVEN spacecraft. $J$. Geophys. Res. Planets, 121, doi:10.1002/2016JE004997.

England, S. L., Liu, G., Kumar, A., Mahaffy, P. R., Elrod, M., Benna, M., et al. (2019). Atmospheric tides at high latitudes in the Martian upper atmosphere observed by MAVEN and MRO. J. Geophys. Res. Space Physics, 124, 2943-2953. https://doi.org/10.1029/2019JA026601 Forbes, J.M., \& M.E. Hagan (2000). Diurnal Kelvin wave in the atmosphere of Mars: Towards an understanding of "stationary density structures observed by the MGS accelerometer. Geophys. Res. Lett., 27, 3563-3566. 
Forbes, J.M., Bridger, A.F.C., Bougher, S.W., Hagan, M.E., Hollingsworth, J.L., Keating, J.M. \& J. Murphy (2002). Nonmigrating tides in the thermosphere of Mars. J. Geophys. Res., 10\%, 5113. doi:10.1029/2001JE001582

Forbes, J.M., Zhang, X., Angelatis i Coll, M., \& G.M. Keating (2004). Nonmigrating tides in the thermosphere of Mars: A quasi-empirical description, Adv. Space Res., 34, 1690-1695.

Forbes, J.M. \& R.A. Vincent (1989). Effects of mean winds and dissipation on the diurnal propagating tide: An analytic approach. Planetary and Space Science, 37, 197-209. doi.org/10.1016/0032-0633(89)90007-X

Forbes, J M. \& Zhang, X. (2018). Polar region variability in the lower thermosphere of Mars from Odyssey and Reconnaissance Orbiter aerobraking measurements. J. Geophys. Res. Space Physics, 123, 8664-8687. https://doi.org/10.1029/2018JA025527

Forbes, J.M., Zhang, X., Maute, A., \& Hagan, M.E. (2018). Zonally symmetric oscillations of the thermosphere at planetary wave periods. J. Geophys. Res. Space Physics, 123, 4110-4128. https://doi.org/10.1002/2018JA025258

Forget, F., Hourdin, F., Fournier, R., Hourdin, C., Talagrand, O., Collins, M., Lewis, S. R., Read, P. L., \& Huot, J.P. (1999). Improved general circulation models of the Martian atmosphere from the surface to above $80 \mathrm{~km}$. J. Geophys. Res., 104, 24155-24175. doi:10.1029/1999JE001025

Forget, F., Montmessin, F., Bertaux, J.-L., González-Galindo, F., Lebonnois, S., Quémerais, E., Reberac, A., Dimarellis, E. \& M.A. López-Valverde (2009). Density and temperatures of the upper Martian atmosphere measured by stellar occultations with Mars Express SPICAM. J. Geophys. Res., 114, E01004, doi:10.1029/2008JE003086 Gilli, G., Forget, F., Spiga, A., Navarro, T., Millour, E., Montabone, L., Kleinböhl, A., Kass, D.M., McCleese, D.J., \& J.T. Schofield (2020). Impact of gravity waves on the middle atmosphere of Mars: A non-orographic gravity wave parameterization based on global climate modeling and MCS observations. J. Geophys. Res., 125, e2018JE005873. doi:10.1029/2018JE005873

González-Galindo, F., Forget, F., Lápez-Valverde, M.A., Angelats i Coll, M. \& E. Millour (2009). A ground-to-exosphere Martian general circulation model: 1. Seasonal, diurnal, and solar cycle variation of thermospheric temperatures. J. Geophys. 
Res., 114, E04001. doi:10.1029/2008JE003246

González-Galindo, F., Chaufray, J.-Y., López-Valverde, M.A., Gilli, G., Forget, F., Leblanc, F., Modolo, R., Hess, S., \& M. Yagi (2013). Three-dimensional Martian ionosphere model: I. The photochemical ionosphere below $180 \mathrm{~km} . J$. Geophys.

Res. Planets, 118, 2105-2123. doi:10.1002/jgre.20150

González-Galindo, F., López-Valverde, M.A., Forget, F., Garciá-Comas, M., Millour, E., \& L. Montabone (2015). Variability of the Martian thermosphere during eight Martian years as simulated by a ground-to-exosphere global circulation model. J. Geophys. Res. Planets, 120, 2020-2035. doi:10.1002/2015JE004925 Greybush, S.J., Kalnay, E., Wilson, R.J., et al. (2019). The Ensemble Mars Atmosphere Reanalysis System (EMARS) Version 1.0. Geosci Data J., 6, 137-150. https://doi.org/10.1002/gdj3.77

Gröller, H., Montmessin, F., Yelle, R.V., Lefvre, F., Forget, F., Schneider, N.M., et al. (2018). MAVEN/IUVS stellar occultation measurements of Mars atmospheric structure and composition. Journal of Geophysical Research: Planets, 123, 14491483. https://doi.org/10.1029/2017JE005466 Guzewich, S.D., Talaat, E.R., \& D.W. Waugh (2012). Observations of planetary waves and nonmigrating tides by the Mars Climate Sounder. J. Geophys. Res., 117, E03010, doi:10.1029/2011JE003924

Hollingsworth, J., \& J.R. Barnes (1996). Forced Stationary Planetary Waves in Mars's Winter Atmosphere. J. Atmos. Sci., 53, 428-448. https://doi.org/10.1175/1520-0469(1996)053¡0428:FSPWIM ¿2.0.CO;2 Huang, C.M., Zhang, S.D. \& Yi, F. (2007). A numerical study of the impact of nonlinearity on the amplitude of the migrating diurnal tide. J. Atmos. Solar-Terr Phys., 69, 631-648.

Joshi, M.M., Hollingsworth, J.L., Haberle, R.M., \& A.F.C. Bridger (2000). An interpretation of Martian thermospheric waves based on analysis of a general circulation model. Geophys. Res. Lett., 27, 613-616.

Kato, S. (1966). Diurnal atmospheric oscillation: 1. Eigenvalues and Hough functions. J. Geophys. Res., 71, 3201-3209. doi:10.1029/JZ071i013p03201

Kleinböhl, A., Schofield, J.T., Kass, D.M., Abdou, W.A., Backus, C.R., Sen, B., Shirley, J.H, Lawson, W.G., Richardson, M.I., Taylor, F.W., Teanby, N.A., \& D.J. McCleese (2009). Mars Climate Sounder limb profile retrieval of atmospheric tem- 
perature, pressure, dust and water ice opacity. J. Geophys. Res., 114, E10006. doi:10.1029/2009JE003358

Kleinböhl, A., Schofield, J.T., Abdou, W.A., Irwin, P.G.J., \& R.J. de Kok (2011). A single-scattering approximation for infrared radiative transfer in limb geometry in the Martian atmosphere. J. Quant. Spectrosc. Radiat. Transfer, 112, 1568-1580. doi:10.1016/j.jqsrt.2011.03.006 Kleinböhl, A., Friedson, A.J., \& J.T. Schofield (2017). Two-dimensional radiative transfer for the retrieval of limb emission measurements in the Martian atmosphere. J. Quant. Spectrosc. Radiat. Transfer, 187, 511-522. doi:10.1016/j.jqsrt.2016.07.009 Kleinböhl, A., Wilson, R.J., Kass, D., Schofield, J.T., \& D.J. McCleese (2013). The semidiurnal tide in the middle atmosphere of Mars. Geophys. Res. Lett., 40, 1952-1959. doi:10.1002/grl.50497 Kleinböhl, A., Schofield, J.T., Kass, D.M., McCleese, D.J., \& S.J. Greybushb (2018),. Mars Atmospheric Profiling from an Orbital Constellation Improving Data Coverage for Mars Data Assimilation, Paper presented at the Mars Atmosphere Data Assimilation Workshop, Le Bourget-du-Lac, France. Available under: http://www-mars.lmd.jussieu.fr/mada2018/Presentations/Poster/ Kleinboehl_poster_MADA2018.pdf Lee, C., et al. (2009). Thermal tides in the Martian middle atmosphere as seen by the Mars Climate Sounder. J. Geophys. Res., 114, E03005, doi:10.1029/2008JE003285

Lindzen, R.S. (1966). On the theory of the diurnal tide. Mon. Wea. Rev., 94, 295301. doi:10.1175/1520-0493(1966)094<0295:OTTOTD $>2.3 . C O ; 2$

Lindzen, R.S. (1968). The application of classical atmospheric tidal theory. Proc. Roy. Soc., A303, 299-316. doi:10.1098/rspa.1968.0052 Lindzen, R.S. (1971). Tides and gravity waves in the upper atmosphere. In Mesospheric Models and Related Experiments, G. Fiocco, ed., D. Reidel Pub., Dordrecht, Holland.

Liu, H.L., McInerney, J.M., Santos, S., Lauritzen, P.H., Taylor, M.A., \& Pedatella, N.M. (2014). Gravity waves simulated by highresolution Whole Atmosphere Community Climate Model. Geophys. Res. Lett., 41, 9106-9112. doi:10.1002/2014GL062468 
Liu, G., England, S., Lillis, R.J.,Mahaffy, P.R., Elrod, M., Benna, M., \& B. Jakosky (2017). Longitudinal structures in Mars upper atmosphere as observed by MAVEN/NGIMS. J. Geophys. Res. Space Physics, 122, 1258-1268, doi:10.1002/2016JA023455

Lo, D.Y., et al. (2015). Non-migrating tides in the Martian atmosphere as observed by MAVEN IUVS. Geophys. Res. Lett., 42, 9057-9063, doi:10.1002/2015GL066268 McCleese, D.J., Schofield, J.T., Taylor, F.W., Calcutt, S.B., Foote, M.C., Kass, D.M., Leovy, C.B., Paige, D.A., Read, P.L. \& R.W. Zurek (2007). Mars Climate Sounder: An investigation of thermal and water vapor structure, dust and condensate distributions in the atmosphere, and energy balance of the polar regions. $J$. Geophys. Res., 112, E05S06, doi:10.1029/2006JE002790

McLandress, C. (2002). The seasonal variation of the propagating diurnal tide in the mesosphere and lower thermosphere: Part II. The role of tidal heating and zonal mean winds J. Atmos. Sci., 59, 907-922.

Medvedev, A.S., Yiğit, E., Hartogh, P., \& Becker, E. (2011). Influence of gravity waves on the Martian atmosphere: General circulation modeling. J. Geophys. Research, 116, E10004. doi:10.1029/2011JE003848

Millour, E., Forget, F., et al. (2018). The Mars Climate Database (Version 5.3). Scientific Workshop: From Mars Express to ExoMars, 2728 February 2018, ESAC Madrid, Spain Moudden, Y., \& J.M. Forbes (2008a), Topographic connections with density waves in Mars aerobraking regime. J. Geophys. Res., 113, E11009. doi:10.1029/2008JE003107 Moudden, Y., \& J.M. Forbes (2008b). Effects of vertically propagating thermal tides on the mean structure and dynamics of Mars lower thermosphere. Geophys. Res. Lett., 35, L23805, doi:10.1029/2008GL036086 Moudden, Y., \& J.M. Forbes (2015). Density prediction in Mars aerobraking region. Space Weather, 13, 86-96, doi:10.1002/2014SW001121 Navarro, T., Madeleine, J.B, Forget, F., Spiga, A., Millour, E., Montmessin, F., \& A. Määttänen (2014). Global climate modeling of the Martian water cycle with improved microphysics and radiatively active water ice clouds. J. Geophys. Res. Planets, 119, 1479-1495, doi:10.1002/2013JE004550 
Navarro, T., Forget, F., Millour, E., Greybush, S.J., Kalnay, E., \& Miyoshi, T. (2017). The challenge of atmospheric data assimilation on Mars. Earth and Space Science, 4, 690-722. https://doi.org/10.1002/2017EA000274

Pedatella, N.M., Raeder, K., Anderson, J.L., \& Liu, H.L. (2014). Ensemble data assimilation in the Whole Atmosphere Community Climate Model. J. Geophys. Res. Atmos., 119, 9793-9809. doi:10.1002/2014JD021776

Pogoreltsev, A.I. (2001). Numerical simulation of secondary planetary waves arising from the nonlinear interaction of the normal atmospheric modes. Phys. Chem. Earth (C), 26, 395-403.

Pogoreltsev, A.I., Vlasov, A.A., Fro'hlich, K., \& Ch. Jacobi (2007). Planetary waves in coupling the lower and upper atmosphere. J. Atmos. Solar-Terr. Phys., $69,, 2083-2101$.

Shaposhnikov, D.S., Medvedev, A.S., Rodin, A.V.V., \& Hartogh, P. (2019). Seasonal water pump in the atmosphere of mars: Vertical transport to the thermosphere. Geophys. Res. Lett., 46, 508-526. https://doi.org/10.1029/2019GL082839 Takahashi, Y.O., J. Fujiwara, and H. Fukunishi, 2006. Vertical and latitudinal structure of the migrating diurnal tide in the Martian atmosphere: Numerical investigations, J. Geophys. Res. 111, E01003, doi:10.1029/2005JE002543 Teitelbaum, H., \& F. Vial (1991). On tidal variability induced by nonlinear interaction with planetary waves. J. Geophys. Res., 96, 14,169-14,178. doi:10.1029/91JA01019

Truskowski, A.O., Forbes, J.M., Zhang, X., \& S.E. Palo (2014). New perspectives on thermosphere tides - 1. Lower thermosphere spectra and seasonal-latitudinal structures. Earth, Planets and Space, 66:136, doi:10.1186/s40623-014-0136-4 Wang, L., D.C. Fritts, \& R.H. Tolson (2006). Nonmigrating tides inferred from the Mars Odyssey and Mars Global Surveyor aerobraking data. Geophys. Res. Lett., 33, L23201. doi:10.1029/2006GL027753 Wang, C., Forget, F., Bertrand, T., Spiga, A., Millour, E., \& Navarro, T. (2018). Parameterization of rocket dust storms on Mars in the LMD Martian GCM: Modeling details and validation. Journal of Geophysical Research: Planets, 123, 9821000. https://doi.org/10.1002/2017JE005255 Wilson, R.J. (2000). Evidence for diurnal period Kelvin waves in the martian atmosphere from Mars Global Surveyor TES data. Geophys. Res. Lett., 27, 3889- 
Wilson, R.J. (2002). Evidence for nonmigrating thermal tides in the Mars upper atmosphere from the Mars Global Surveyor Accelerometer Experiment. Geophys. Res. Lett., 29, 1120. doi:10.1029/2001GL013975

Wilson, R.J., Neumann, G., \& M.D. Smith (2007). The diurnal variation and radiative influence of Martian water ice clouds. Geophys. Res. Lett., 34, L02710. doi:10.1029/2006GL027976

Wilson, R.J., Lewis, S.R., Montabone, L. \& M.D. Smith (2008). Influence of water ice clouds on Martian tropical atmospheric temperatures. Geophys. Res. Lett., 35, L07202, doi:10.1029/2007GL032405

Wilson, R.J., \& S.D. Guzewich (2014). Influence of water ice clouds on nighttime tropical temperature structure as seen by the Mars Climate Sounder. Geophys.

Res. Lett., 41, 3375-3381. doi:10.1002/2014GL060086

Wilson, R.J., Millour, E., Navarro, T., Forget, F. \& M.A. Kahre (2014). GCM simulations of aphelion season tropical cloud and temperature structure, in Mars Atmosphere: Modeling and Observations, 5th International Workshop, Oxford, UK. http://wwwmars.lmd.jussieu.fr/oxford2014/abstracts/wilson_clouds_oxford2014.pdf. Withers, P.G. (2006). Mars Global Surveyor and Mars Odyssey Accelerometer observations of the Martian upper atmosphere during aerobraking. Geophys. Res. Lett., 33, L02201, doi:10.1029/2005GL024447

Withers, P.G., Bougher, S.W., \& G.M. Keating (2003). The effects of topographically controlled thermal tides in the Martian upper atmosphere as seen by the MGS Accelerometer. Icarus, 164, 14-32.

Withers, P., Pratt, R., Bertaux, J.L., \& Montmessin, F. (2011). Observations of thermal tides in the middle atmosphere of Mars by the SPICAM instrument. $J$. Geophys. Res., 116, E11005, doi:10.1029/2011JE003847.

Wu, D-H., Miyahara, S. \& Y. Miyoshi (1989). A nonlinear simulation of the thermal diurnal tide, J. Atmos. Solar-Terr. Phys., 51, 1017-1030, doi.org/10.1016/0021-9169(89)90017-2

Wu, Z., T. Li, \& X. Dou (2015), Seasonal variation of Martian middle atmosphere tides observed by the Mars Climate Sounder, J. Geophys. Res. Planets, 120, 22062223, doi:10.1002/2015JE004922 
Zurek, R.W. (1976). Diurnal tide in the Martian atmosphere. J. Atmos. Sci., 33, $1281 \quad 321-337$.

${ }_{1282}$ Zurek, R.W. (1988). Free and forced modes in the Martian atmosphere. J. Geo${ }_{1283} \quad$ phys. Res., 93, 9452-9462.
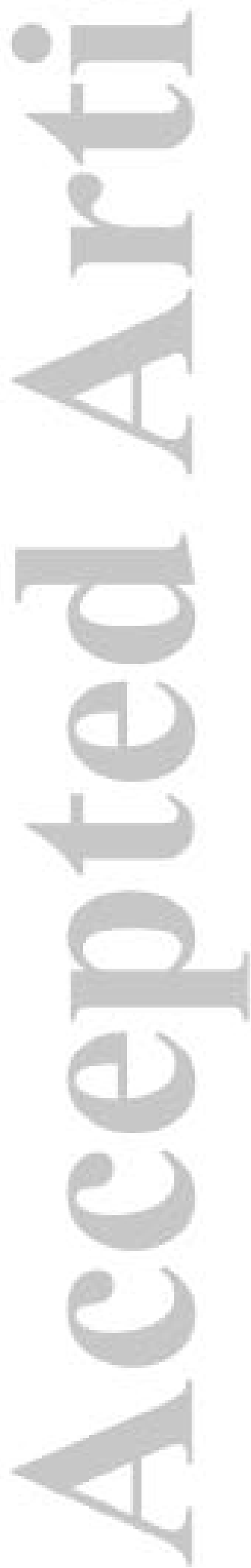


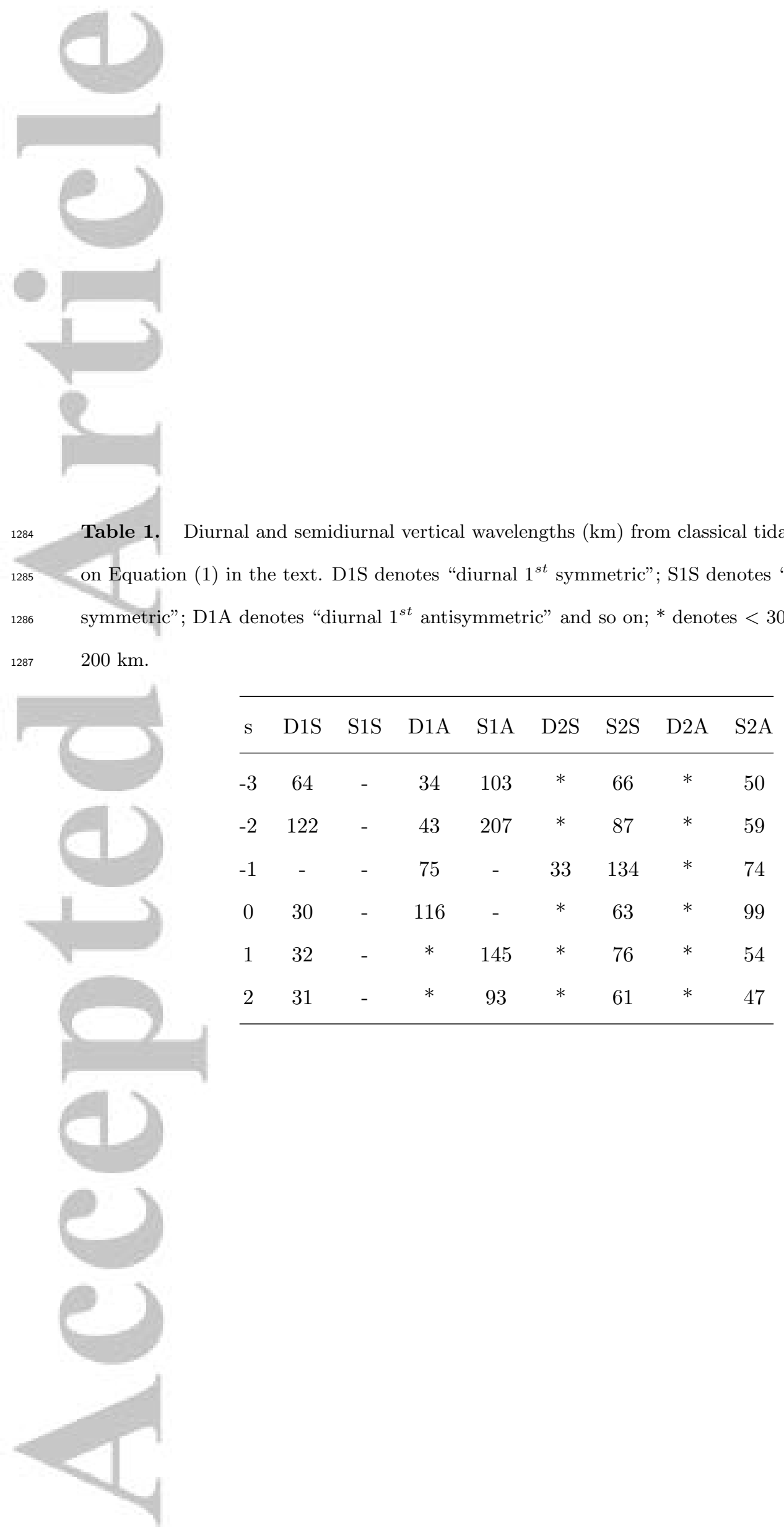


Figure 1.
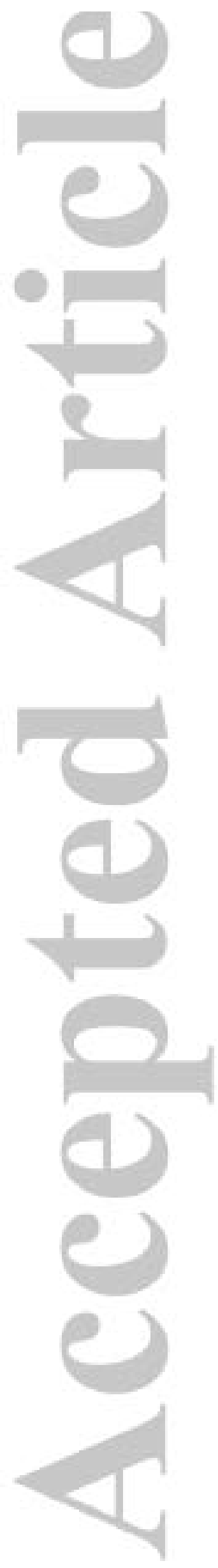

(C2020 American Geophysical Union. All rights reserved. 
Figure 2.
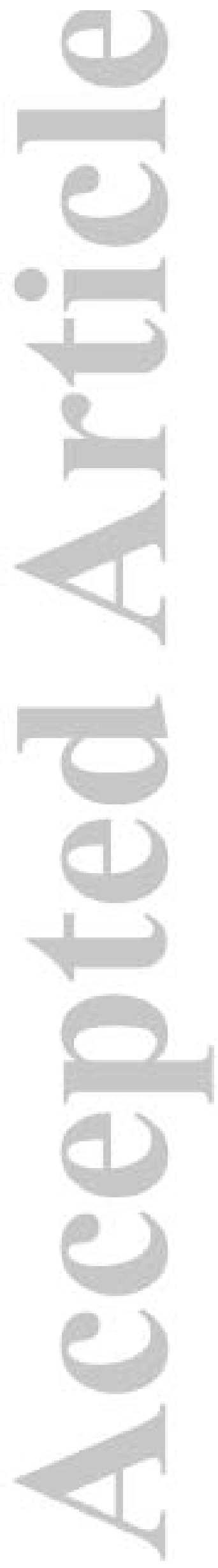

(C2020 American Geophysical Union. All rights reserved. 

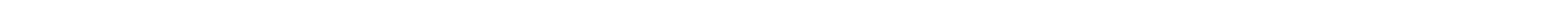
Figure 3.
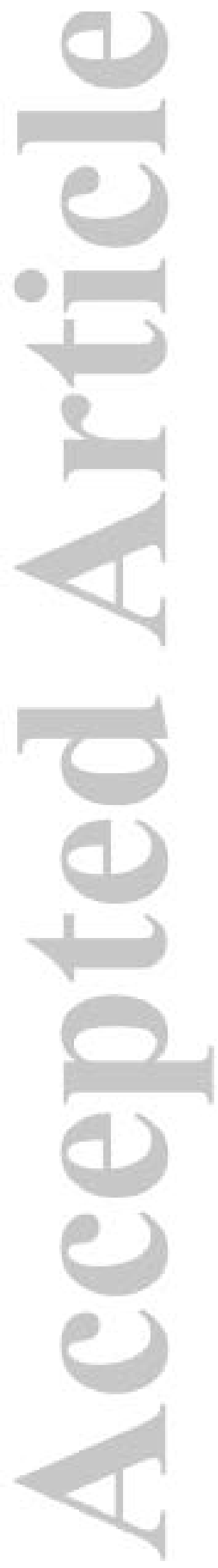

(C2020 American Geophysical Union. All rights reserved. 


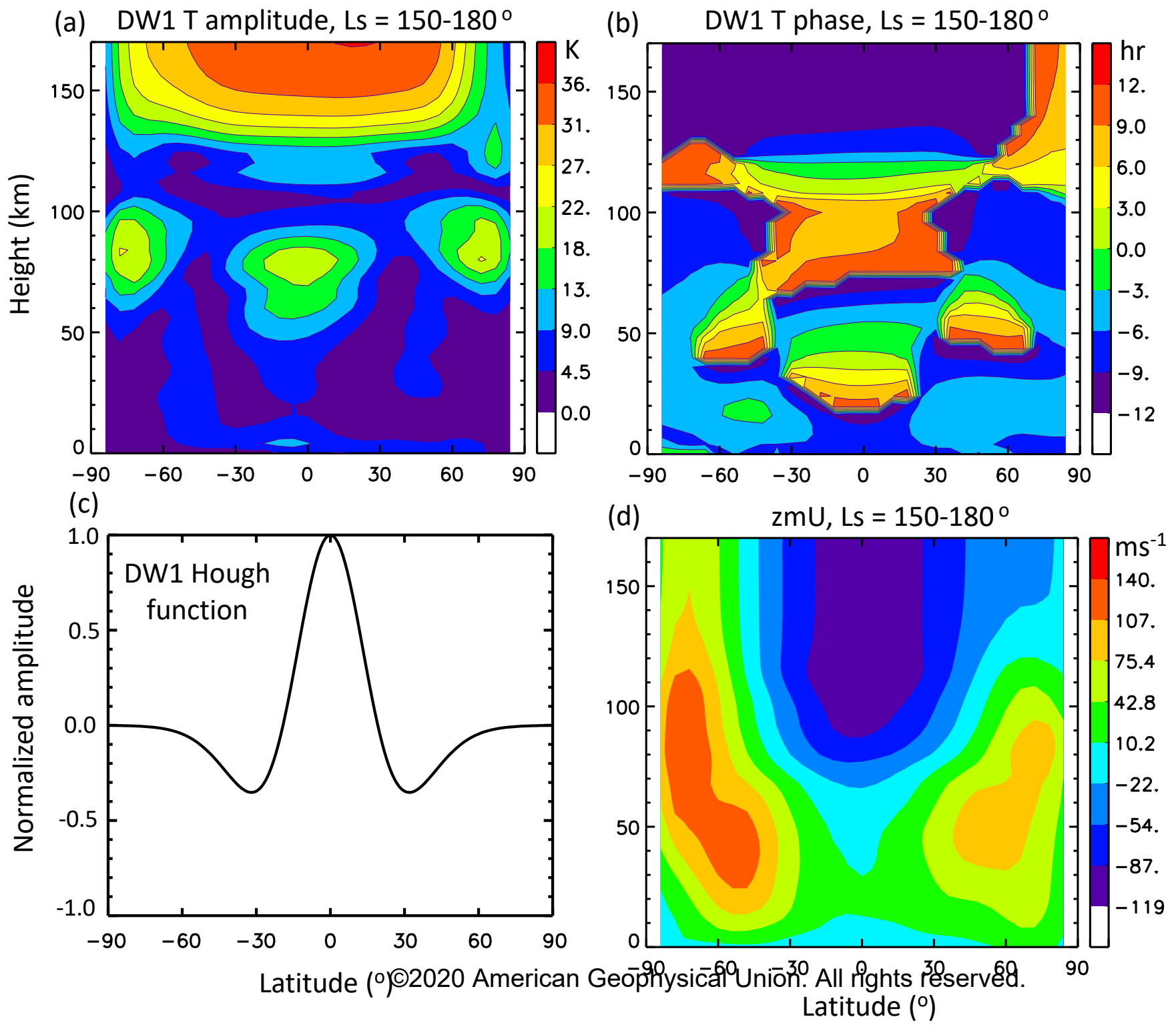


Figure 4.
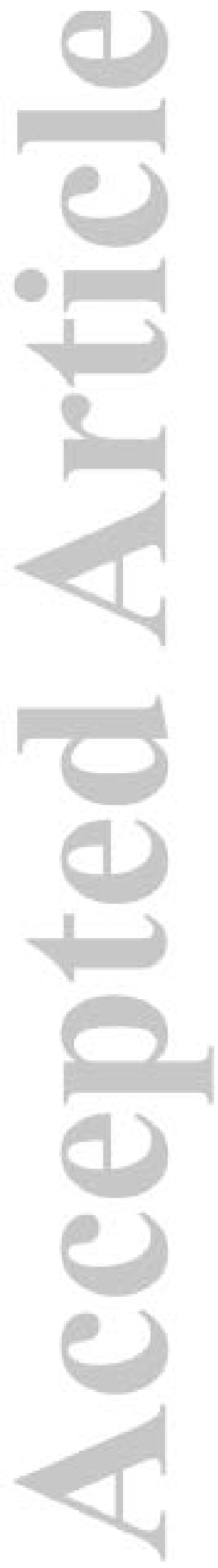

(C2020 American Geophysical Union. All rights reserved. 
Figure 5.
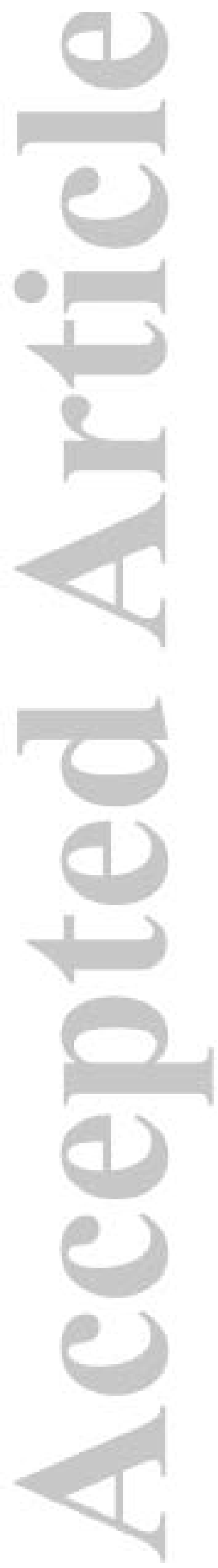

(C2020 American Geophysical Union. All rights reserved. 


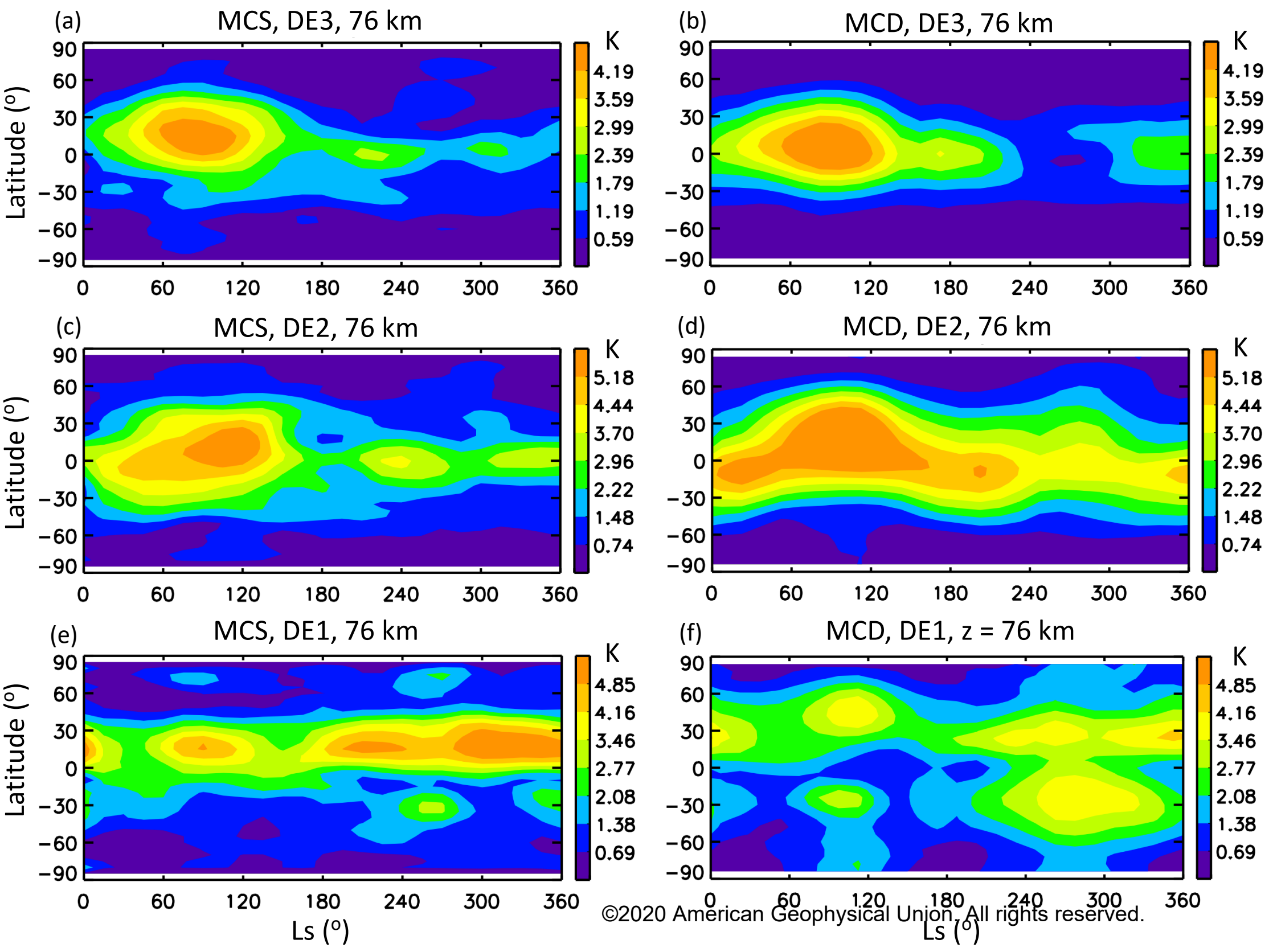


Figure 6.
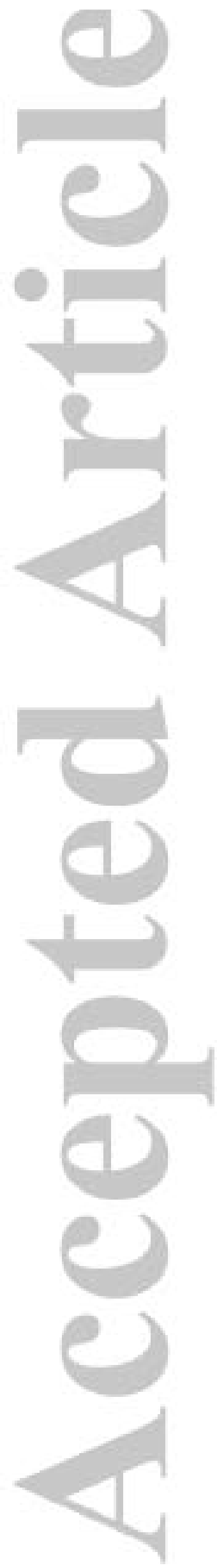

(C2020 American Geophysical Union. All rights reserved. 
Figure 7.
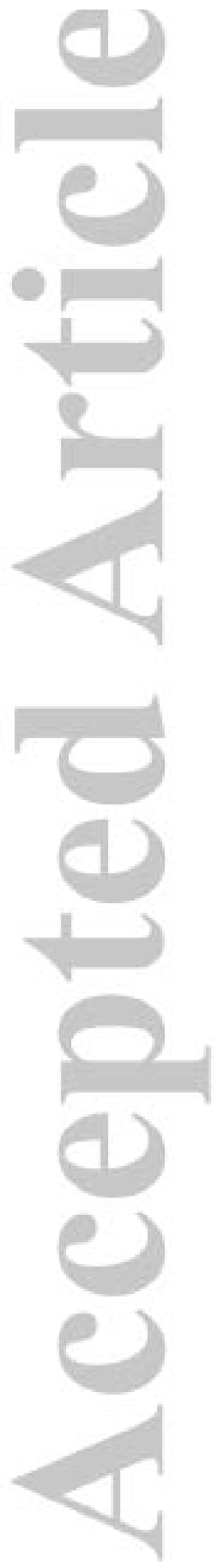

(C2020 American Geophysical Union. All rights reserved. 
Figure 8.
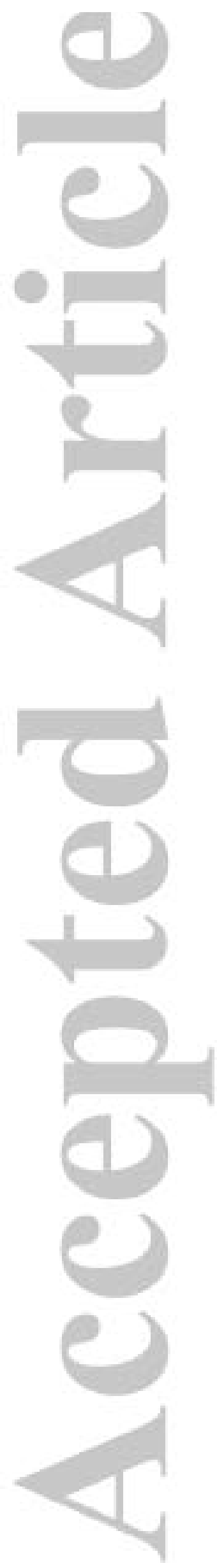

(C2020 American Geophysical Union. All rights reserved. 


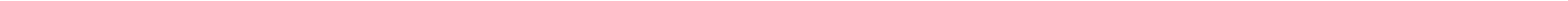


Figure 9.
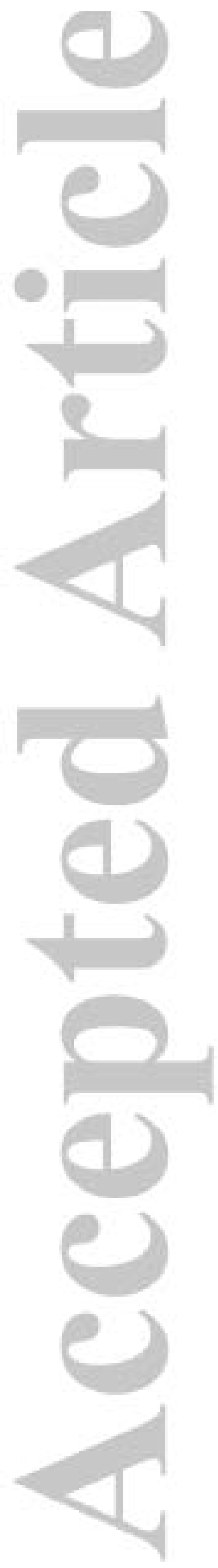

(C2020 American Geophysical Union. All rights reserved. 
Figure 10.
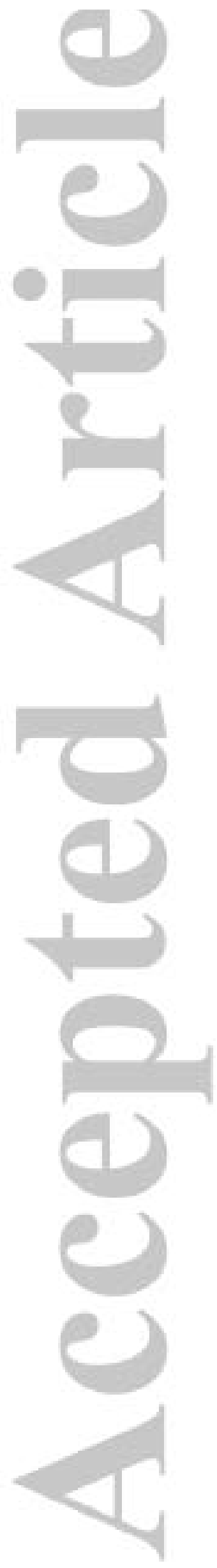

CC2020 American Geophysical Union. All rights reserved. 
(a)

SPW1, Ls 60-90०
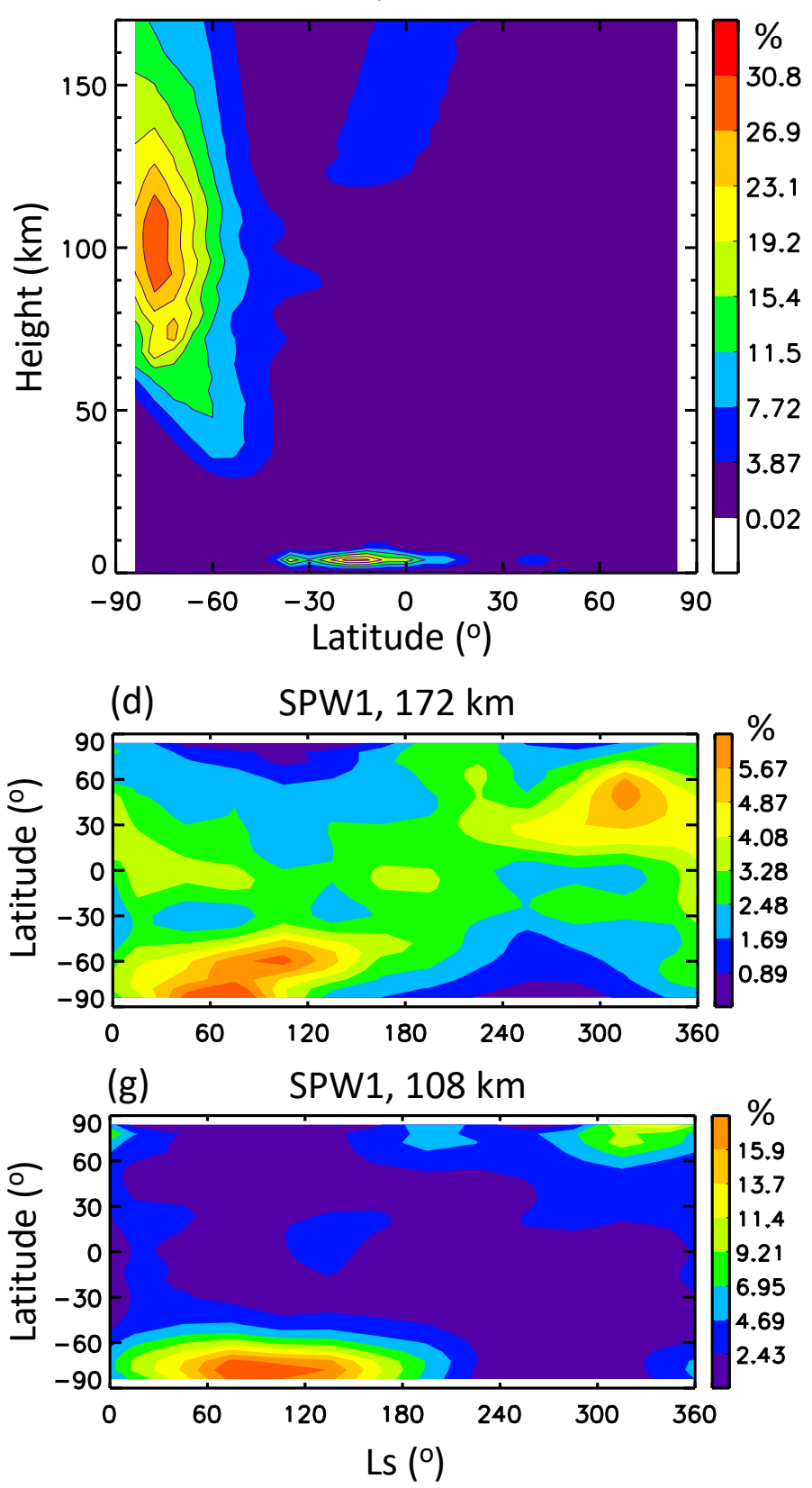

(b) SPW2, Ls 60-90०
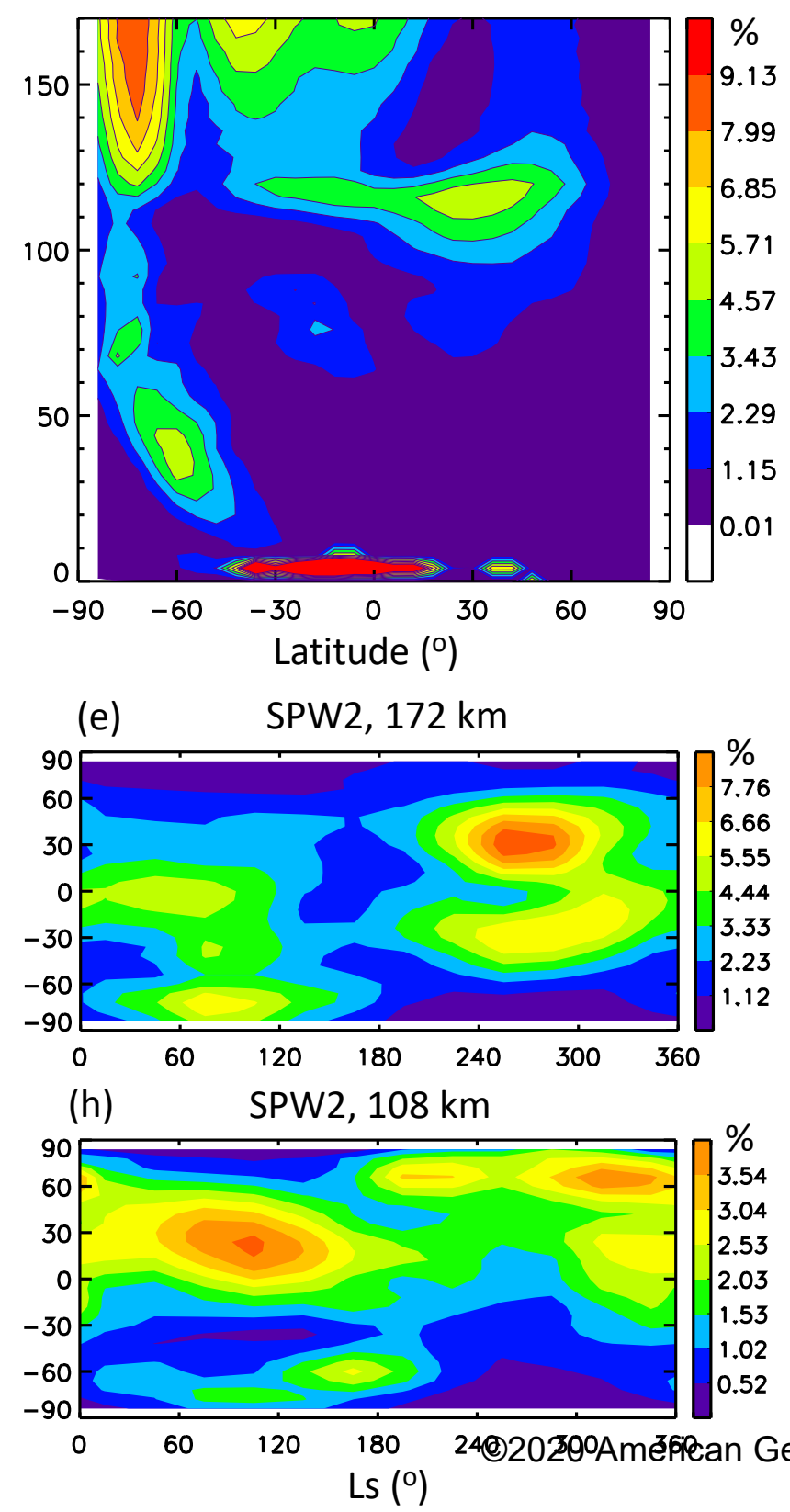

(c)

SPW3, Ls 60-90

Figure 11.
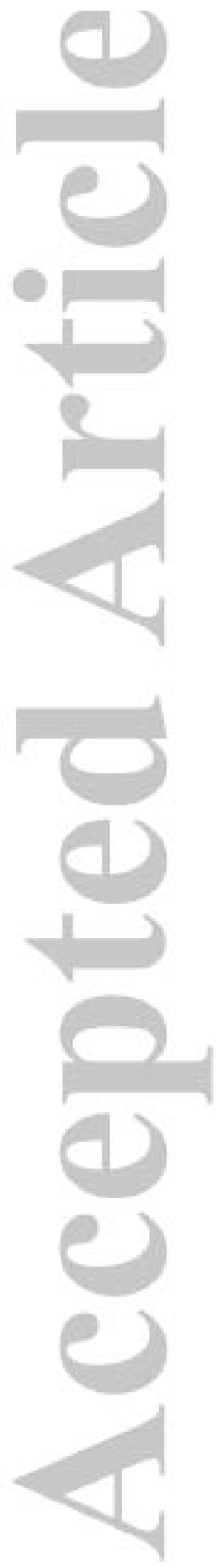

CC2020 American Geophysical Union. All rights reserved. 
Figure 12.


(C2020 American Geophysical Union. All rights reserved. 
Figure 13.
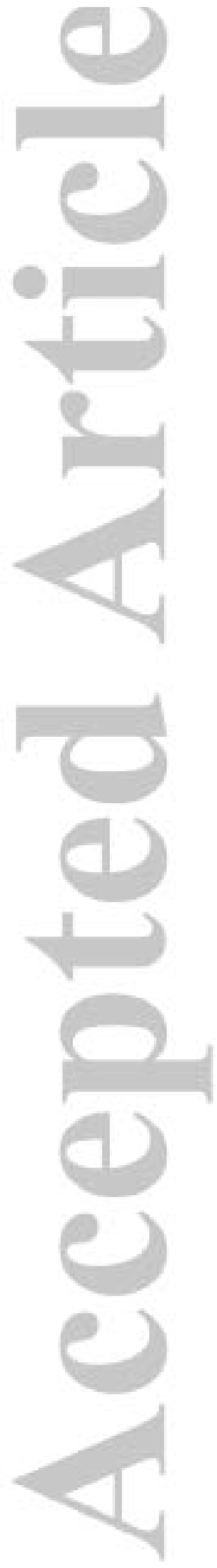

(C2020 American Geophysical Union. All rights reserved. 
(a)

MGS, Wave 1

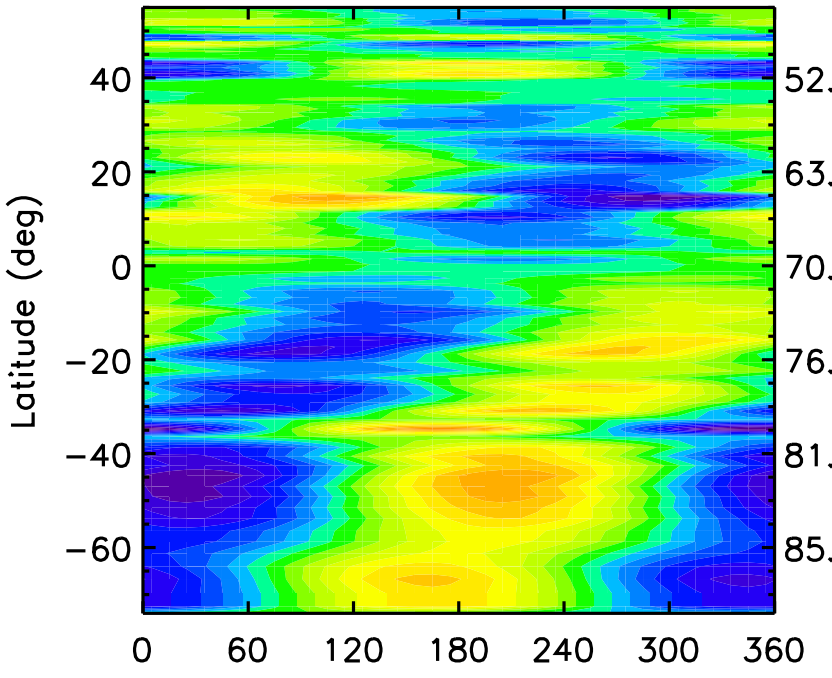

(d) MGS, Wave 2

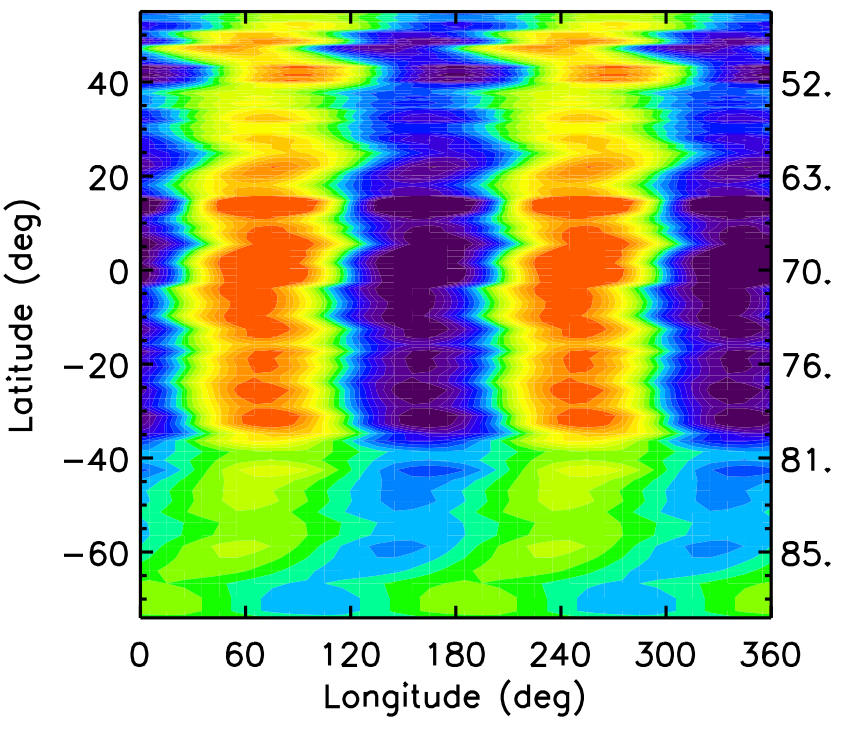

(b) sampled MCD, Wave 1

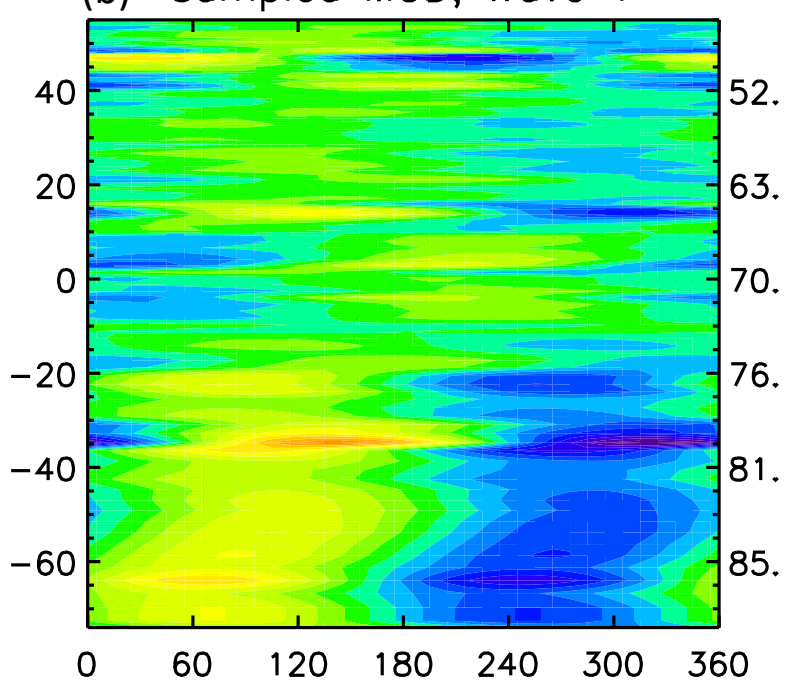

(e) sampled MCD, Wave 2

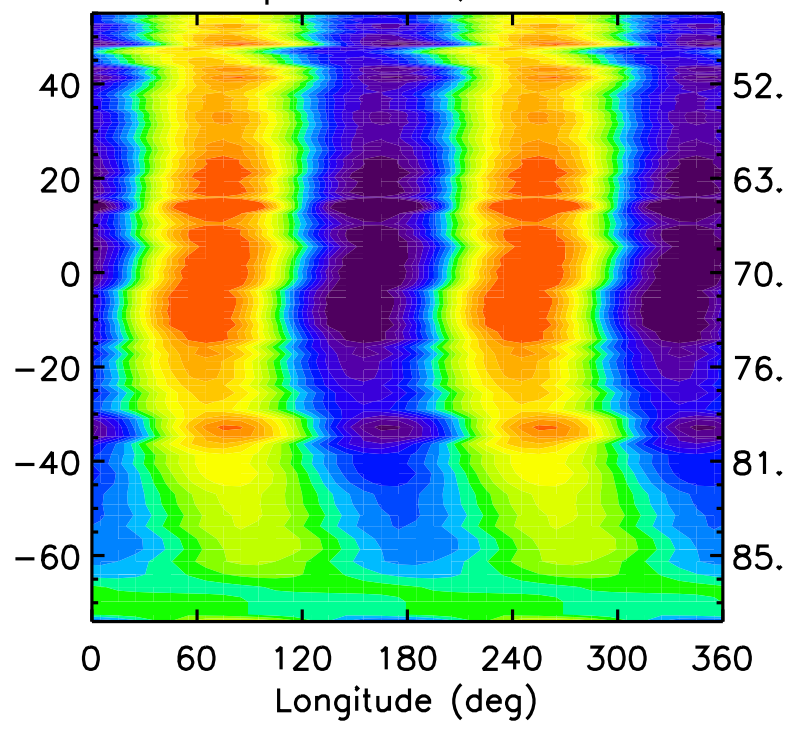

(c)

full MCD, Wave 1
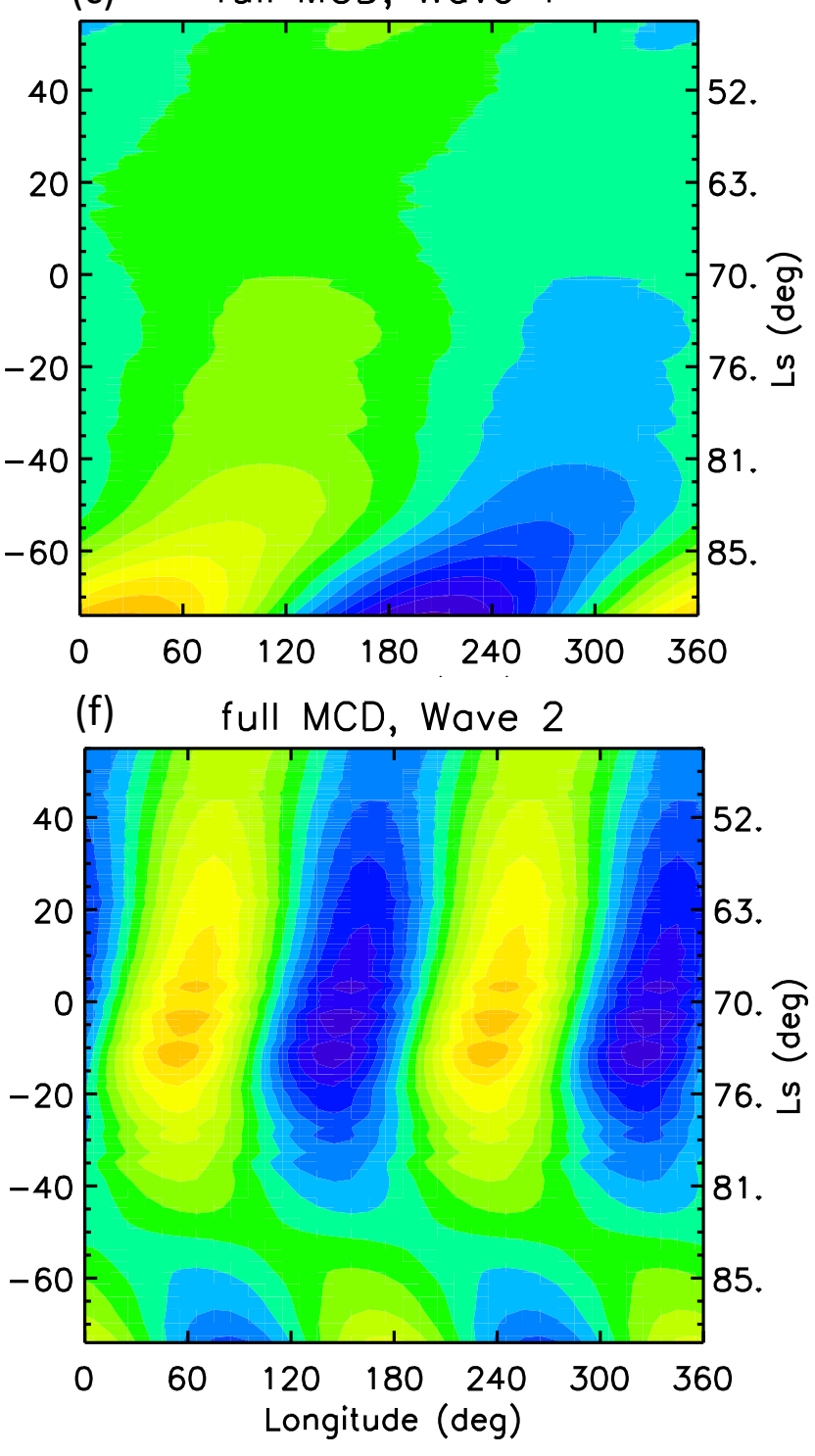

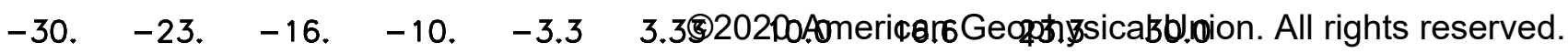
Phase II Relative Density Residual (\%) 
Figure 14.
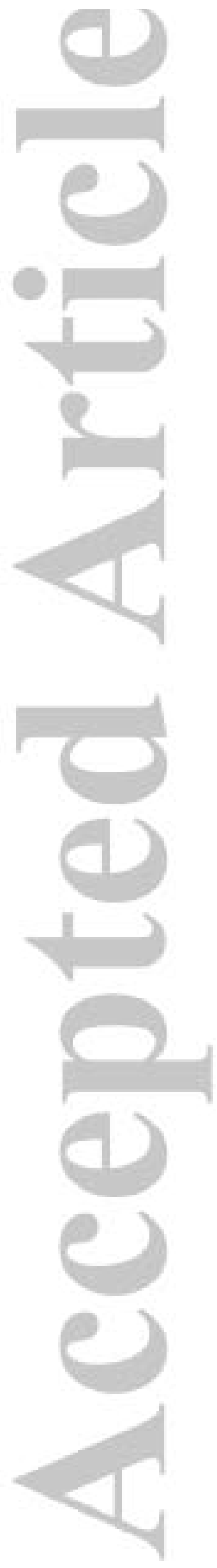

(C2020 American Geophysical Union. All rights reserved. 
MGS, Wave 3

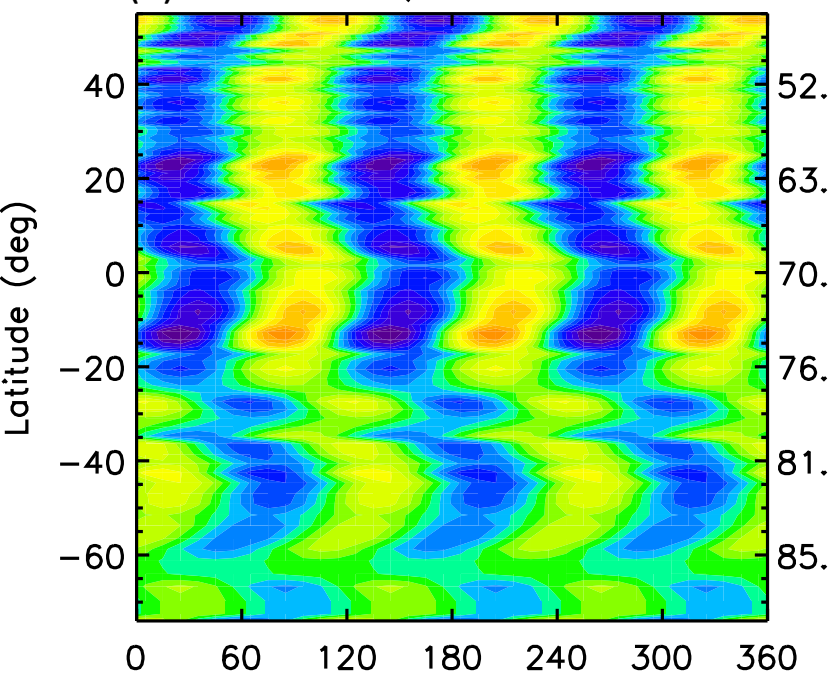

(d) MGS, Wave 4

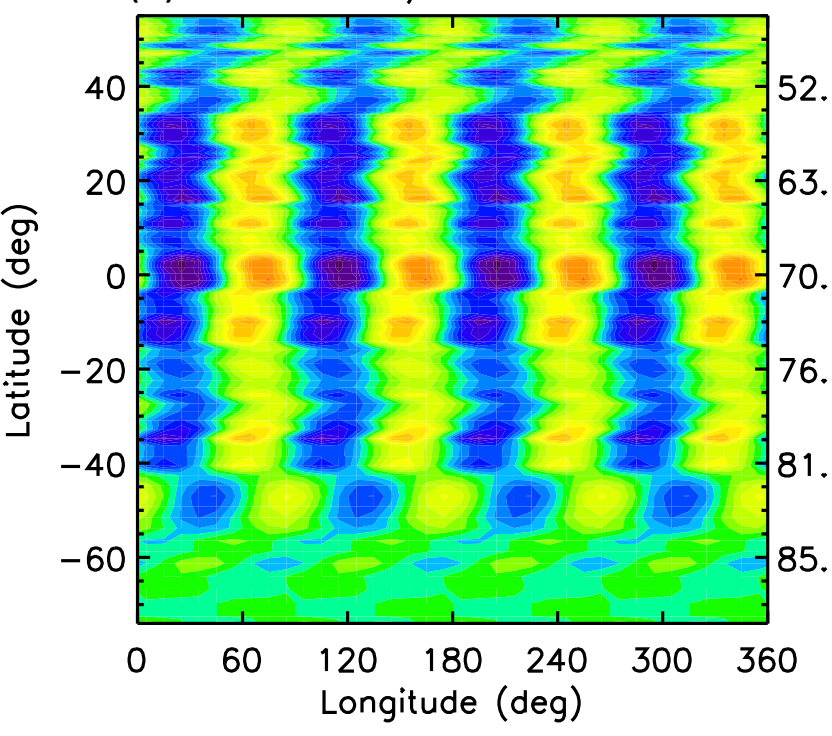

(b) sampled MCD, Wave 3

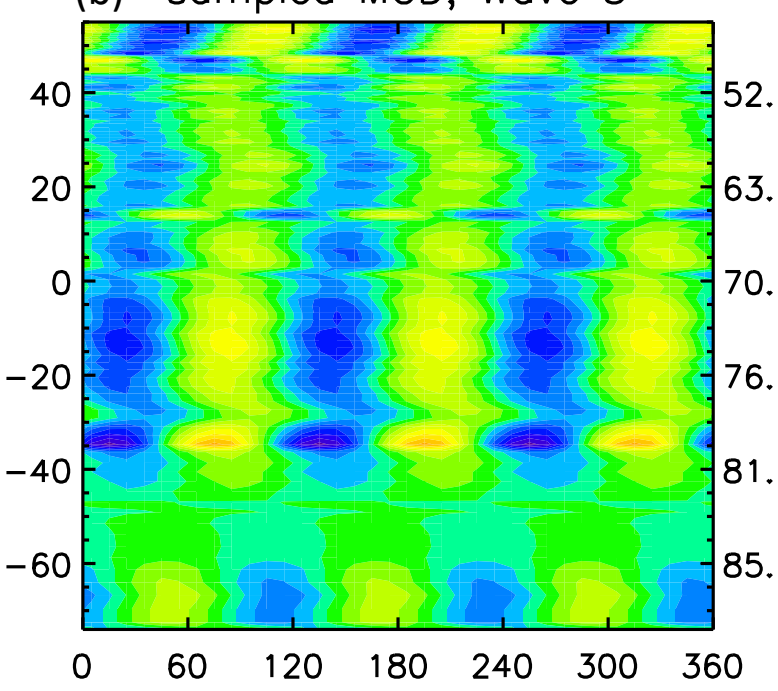

(e) sampled MCD, Wave 4

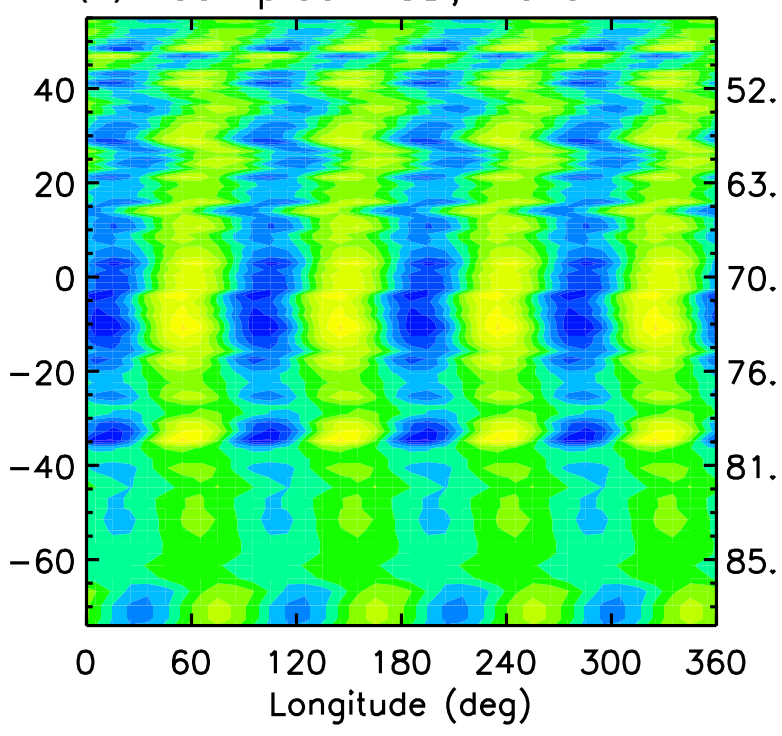

(c) full MCD, Wave 3


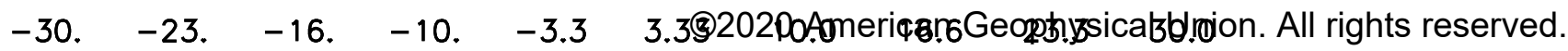
Phase II Relative Density Residual (\%) 